\title{
Coherent structure generation in near-wall turbulence
}

\author{
By W. SCHOPPA AND F. HUSSAIN \\ Department of Mechanical Engineering, University of Houston, Houston, TX 77204, USA
}

(Received 11 August 2000 and in revised form 23 July 2001)

We present a new mechanism for generation of near-wall streamwise vortices - which dominate turbulence phenomena in boundary layers - using linear perturbation analysis and direct numerical simulations of turbulent channel flow. The base flow, consisting of the mean velocity profile and low-speed streaks (free from any initial vortices), is shown to be linearly unstable to sinuous normal modes only for relatively strong streaks, i.e. for wall inclination angles of streak vortex lines exceeding $50^{\circ}$. Analysis of streaks extracted from fully developed near-wall turbulence indicates that about $20 \%$ of streak regions in the buffer layer exceed the strength threshold for instability. More importantly, these unstable streaks exhibit only moderate (twofold) normalmode amplification, the growth being arrested by self-annihilation of streak-flank normal vorticity due to viscous cross-diffusion. We present here an alternative, streak transient growth (STG) mechanism, capable of producing much larger (tenfold) linear amplification of $x$-dependent disturbances. Note the distinction of STG-responsible for perturbation growth on a streak velocity distribution $U(y, z)$-from prior transient growth analyses of the (streakless) mean velocity $U(y)$. We reveal that streamwise vortices are generated from the more numerous normal-mode-stable streaks, via a new STG-based scenario: (i) transient growth of perturbations leading to formation of a sheet of streamwise vorticity $\omega_{x}$ (by a 'shearing' mechanism of vorticity generation), (ii) growth of sinuous streak waviness and hence $\partial u / \partial x$ as STG reaches nonlinear amplitude, and (iii) the $\omega_{x}$ sheet's collapse via stretching by $\partial u / \partial x$ (rather than rollup) into streamwise vortices. Significantly, the three-dimensional features of the (instantaneous) streamwise vortices of $x$-alternating sign generated by STG agree well with the (ensemble-averaged) coherent structures educed from fully turbulent flow. The STGinduced formation of internal shear layers, along with quadrant Reynolds stresses and other turbulence measures, also agree well with fully developed turbulence. Results indicate the prominent-possibly dominant-role of this new, transient-growth-based vortex generation scenario, and suggest interesting possibilities for robust control of drag and heat transfer.

\section{Introduction}

Recurring organized large-scale events in turbulent boundary layers have been studied extensively for over five decades (Townsend 1956; Kline et al. 1967; Kovasznay, Kibens \& Blackwelder 1970; Cantwell 1981; Panton 1997). Nevertheless, the generation mechanism and evolutionary dynamics of near-wall coherent structures (CS) are poorly understood - a critical barrier to possible robust modelling and control of drag (as well as heat and mass transfer) in turbulent boundary layers. The dominant role of streamwise vortices near the wall in turbulence production and drag 


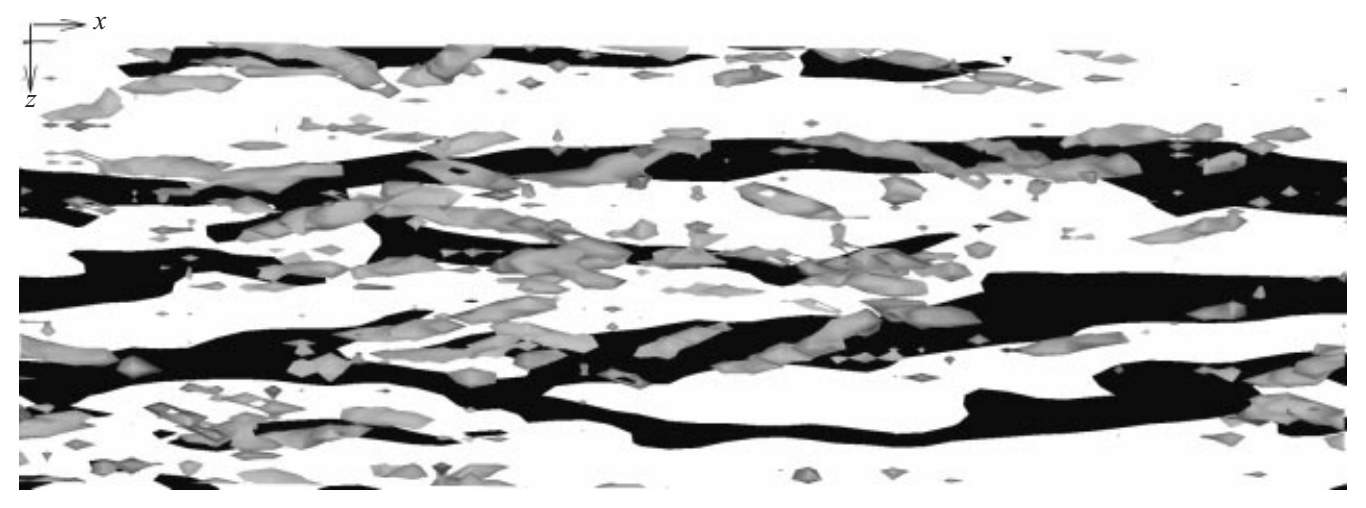

FiguRE 1. Top view of the near-wall region covering $\left(x^{+}, z^{+}\right)=(1400,450)$ in the streamwise and spanwise directions. Lifted low-speed streaks (black) denote $u^{\prime}<0$ at $y^{+}=20$ and streamwise vortices (grey-shaded) are indicated by the $\lambda_{2}$ vortex definition (Jeong \& Hussain 1995) in the region $0<y^{+}<60$.

generation is now widely accepted (e.g. Kim, Moin \& Moser 1987). Although the outer layer also contains energetic (larger) structures (Adrian, Meinhart \& Tomkins 1999), recent 'numerical experiments' (Jimenez \& Pinelli 1999) confirm that the essential inner-layer dynamics (namely $y^{+}<60$ ) can operate autonomously. Note that this claim is asserted in several early works (e.g. Kline et al. 1967), in contrast to studies which emphasize coupling of near-wall and outer-layer events (e.g. Rao, Narasimha \& Narayanan 1971). The dominance of streamwise vortices is supported quantitatively by the educed (i.e. ensemble-averaged) near-wall CS, whose model (Jeong \& Hussain 1992; Jeong et al. 1997) captures well the documented near-wall features. Clearly, a formidable challenge is to identify and explain the prevalent dynamics of streamwise CS generation and evolution near the wall-the key to modelling and control of turbulent boundary layers.

A representative snapshot of fully developed near-wall turbulence (figure 1) illustrates the dominant structural features, i.e. streamwise vortices and elongated 'streaks' of relatively lower-speed and less-stirred fluid. We reiterate that the socalled 'bursting' - used to describe the intermittent, energetic process perceived from scalar markers in flow visualization or from stationary sensors-does not reflect any particular event, but is primarily the consequence of passage of near-wall vortices. Hence, 'bursting' as well as the more meaningful 'sweep' and 'ejection' events can at most be indirect indicators of streaks, as streaks can exist without streamwise vortices (see figure 1) and hence exhibit no energetic process. Note also the distinction between lifted streaks - regions of $u<0$ in the buffer region-and ejections where both $u<0$ and $v>0$ occur simultaneously $(u, v$, and $w$ denoting streamwise $(x)$, wall-normal $(y)$, and spanwise $(z)$ velocity fluctuations). Physically, a lifted low-speed streak is the result of ejection during passage of a previous streamwise vortex (or vortices). Furthermore, ejections are actually long-lived as they advect several hundred wall units downstream (Johansson, Alfredsson \& Kim 1991). Robinson (1991) concludes that advecting streamwise vortices eject low-speed fluid which, left behind, creates elongated streaks. An additional connection between near-wall vortices and streaks explored here is that new vortices are, in turn, generated via growth of streak perturbations.

The central issue addressed herein is: how are streamwise vortices generated? As reviewed below, numerous widely disparate mechanisms for vortex formation 
(frequently called 'regeneration') have been developed, many quite plausible and even fairly self-consistent. For a comprehensive collection of proposed mechanisms, see Panton (1997). Of the numerous proposed regeneration mechanisms, most fall into one of two broad categories: (i) parent-offspring scenarios, and (ii) instability-based mechanisms. We characterize the first as the generation of new vortices by the direct action (induction) of existing vortices. In contrast, the second scenario involves local instability of a quasi-steady base flow, without requiring the presence of parent vortices. Note that recurring instability indeed requires a 'feedback' mechanism, by which previous events generate an (longer timescale) unstable base flow on which (shorter timescale) perturbations can grow; thus, prior vortices play only an indirect role. We will show that near-wall CS (re)generation results from a distinct mechanismstreak transient growth-which unites elements of parent-offspring and instabilitybased scenarios, and is far more prevalent and energetic than normal-mode instability. In this scenario, viscosity comes into play not through wall effects (diffusion as well as no-slip), but instead indirectly through vorticity self-annihilation across a streak flanked by opposite-signed wall-normal vorticity.

\subsection{Parent-offspring regeneration}

\subsubsection{Hairpin vortex formation}

The widely cited concept of a 'hairpin' vortex, initiated from a conceptual model of Theodorsen (1952), refers to an $\Omega$-shaped vortical structure with two streamwiseoriented legs connected via a raised, spanwise-oriented arch. Hairpin vortices have been analysed extensively, both computationally (Moin, Leonard \& Kim 1986; Singer 1996), and experimentally in flow visualization studies (Gad-el-Hak \& Hussain 1986; Haidari \& Smith 1994) and in (x,y)-plane PIV measurements (Adrian et al. 1999). There is, however, considerable disagreement as to the underlying hairpin vortex generation mechanism.

Smith \& Walker (1994) propose a regeneration scenario in which parent hairpin vortices spawn offspring hairpins, both behind the (spanwise) head (or 'arch') and beside each of the (streamwise) legs. In this scenario, vortex formation is driven by unsteady separation near the wall (Doligalski \& Walker 1984), in which the parent hairpin produces localized ejections near its head and legs, the resulting inflectional shear flow then rolling up by Kelvin-Helmholtz instability and giving birth to new hairpins. In contrast, in DNS initialized with conditionally averaged streamwise vortices, Zhou et al. (1999) reveal that a sufficiently strong single hairpin can generate a packet of hairpin vortices, both upstream and downstream of the parent hairpin. In this mechanism, the induction of the parent vortex generates intense local shear layers, composed predominantly of spanwise vorticity. Subsequently, these shear layers rollup into arch vortices which link up with the existing streamwise-oriented legs, and are stretched by the mean shear into offspring hairpin vortices, detached from the primary hairpin vortex. These observations are also consistent with the conditional-average initial condition studied by Kim (1987), who emphasized the role of localized vorticity stretching above the parent legs in generating the offspring arch vortex. Other studies (discussed in §1.2) propose that a local Kelvin-Helmholtz-type instability generates hairpins through roll-up of the crest of a lifted streak.

The vortex surface geometry in these idealized flows exhibiting hairpin vortex formation is characterized by an $x$-localized, lifted low-speed streak. Clearly, the vortex lines in the lifted region will tilt forward, due to the faster advection of their crests across a shear, much like the Lin \& Corcos (1984) mechanism in mixing layers. Subsequently, the vortex sheet consisting of these tilted vortex lines will collapse 
due to sustained stretching by the mean shear, generating a hairpin vortex with two well-defined legs. In each of the hairpin vortex studies cited above, such $x$-localized lifted streaks are generated externally, whereas low-speed streaks in fully developed near-wall turbulence are highly elongated and do not experience the inherent tilting of $x$-localized streaks. In particular, for the case of an $x$-independent streak (as a model of observed highly elongated streaks), vortex lines coincide with the local $U$ isocontours (bell-shaped in the $(y, z)$-plane, not to be confused with isovelocity lines in the mean flow $U(y))$. Hence, for $x$-independent streaks, vortex lines experience no tilting and thus no $\omega_{x}$ amplification, due to the fact that $U=$ constant along each streak vortex line.

Note that hairpin vortices with both legs extending into the near-wall region are actually rare in near-wall turbulence, both in instantaneous realizations (Robinson 1991; Brooke \& Hanratty 1993) and in the ensemble-averaged CS (Jeong et al. 1997). Our results (Schoppa \& Hussain 1997, hereinafter referred to as SH) indicate that the generation dynamics of individual streamwise vortices are distinctly different from those of true (two-legged) hairpins. In DNS of blowing-initiated (initially spanwise symmetric) turbulent spots, individual (spanwise asymmetric) streamwise vortices become more numerous and intense than hairpins (Singer 1996), suggesting that the initial hairpin generation observed in idealized studies is not sustained far downstream (near the wall). Additionally, Zhou et al. (1999) have observed that asymmetric hairpins (i.e. 'hooks' with only one leg) grow more rapidly than symmetric ones.

\subsection{2. $\omega_{x}$ sheet generation and roll-up}

An alternative parent-offspring scenario is proposed by Brooke \& Hanratty (1993), who studied the spatiotemporal velocity field from DNS data, but found no hairpintype vortices. They propose that an opposite-signed offspring vortex forms immediately underneath a parent vortex, whose downstream end has lifted from the wall. By identifying streamwise vortices as centres in instantaneous $(v, w)$ vector patterns in successive $(y, z)$-planes, Bernard, Thomas \& Handler (1993) independently obtained similar findings, further noting that new vortices tend to form from strong $\omega_{y}$, typically on the sweep side of the parent vortex. Brooke \& Hanratty demonstrate that the production of offspring $\omega_{x}$ is dominated by the vorticity generation term $-(\partial w / \partial x)(\partial u / \partial y)$, where $\partial w / \partial x$ (hence $\left.\omega_{y}\right)$ is generated by the parent vortex inclination to the wall. Note, however, that this term tends to produce thin sheets of $\omega_{x}$, rather than vortices, as demonstrated herein. Vortex formation from such near-wall $\omega_{x}$ sheets is often attributed to sheet rollup via two-dimensional self-advection. Note that in two-dimensional flow, $\omega_{x}$ sheet rollup can occur via two distinct mechanisms: (i) dipole-like head-tail formation due to the wall image vorticity (Jimenez \& Orlandi 1993), or (ii) lifting of the wall-generated $\omega_{x}$ (due to no-slip) by a parent vortex, as in vortex wall-rebound (Orlandi 1990).

While $x$-overlapping of streamwise vortices has been perceived as evidence of a parent $\rightarrow$ offspring causation, the question remains: are parent vortices required for offspring generation? Our prior results $(\mathrm{SH})$ show that vortex formation can in fact occur in the absence of a parent vortex, in which pairs of partially overlapping vortices are generated simultaneously by growth of streak perturbations. Further, analysis of $\omega_{x}$ advection terms (discussed herein) indicates that neither of the (two-dimensional) $\omega_{x}$ sheet rollup mechanisms is relevant to the vortex generation process described here. In contrast to the limitations of such two-dimensional rollup mechanisms, the 
vortex generation is inherently three-dimensional, driven by localized stretching and eventual collapse of $\omega_{x}$ sheets.

\subsection{Instability-based regeneration}

Unlike in parent-offspring regeneration scenarios, existing vortices may alternatively play a more indirect role, by generating and leaving behind an unstable base flow (via their induction), which in turn spawns new vortices-a kind of 'feedback'. Not surprisingly, there is considerable variation among the proposed mechanisms of instability and feedback, including centrifugal instability, wave-shear instability, oblique mode interaction of the turbulent mean profile, and (local) shear-layer-type streak instabilities.

\subsubsection{Centrifugal and wave-shear instabilities}

Several studies suggest that streamwise vortices originate from locally curved streamlines generated near the wall. For example, the condition for Taylor-Görtler instability - sufficient concave curvature of near-wall streamlines-is locally satisfied above $y^{+} \sim 50$ (Brown \& Thomas 1977). Alternatively, Phillips, Wu \& Lumley (1996) consider a Craik-Leibovich (type 2) instability mechanism, involving $x$-dependent perturbation growth on shear flows with small-amplitude streamwise undulation. In particular, they present evidence of Craik-Leibovich-based longitudinal vortex formation near a (rigid) wavy wall, suggested to be locally representative as well of the fluctuating streamwise velocity field in near-wall turbulence. While base flows with streamline curvature or streamwise waviness can conceivably be produced by the induction by (outer) spanwise vortices, it is unclear how the instability-generated streamwise vortices might do so. The necessary feedback mechanism for successive episodes of instability and vortex generation is thus not evident. In contrast to the $z$-independent base flows of these studies, low-speed streaks with strong $z$-dependence are prominent and themselves susceptible to a different (inherently three-dimensional) perturbation growth mechanism, discussed herein. Further it is difficult to isolate the role of centrifugal and wave-shear instabilities, as the geometry of streamwise vortices arising (upon nonlinear evolution) from these instabilities has not been compared with instantaneous structures in near-wall turbulence. Note that the predominance of the alternative transient-growth-based scenario developed here is clearly demonstrated by such a comparison, as the resulting instantaneous vortices correspond closely to the near-wall CS.

\subsubsection{Oblique mode interaction}

In early theoretical studies of shear layer transition, Benney (1961) addressed the generation of $x$-elongated regions of longitudinal vorticity as a mechanism of transition to small-scale turbulence. For the case of a $\tanh U(y)$ mixing layer, Benney demonstrates that the generation of an $x$-mean secondary flow is inherent to the nonlinear interaction of three-dimensional oblique modes. Note that his focus is on growth of second-order perturbations by the nonlinear interaction of (initially unstable) primary oblique modes. The secondary motion is shown to consist of four counter-rotating cells of longitudinal vorticity per $z$-wavelength, implying the generation of spanwise-alternating streamwise 'vortices'. Although generation of $x$ mean perturbation modes is a common feature of nonlinear mode interactions in shear flows, the mixing-layer dynamics outlined by Benney are not applicable to the growth of linear streak perturbations in turbulent boundary layers, analysed herein. First, streak instability upon nonlinear evolution similarly generates $x$-averaged streamwise 
vorticity (Hamilton, Kim \& Waleffe 1995; SH), but the underlying physical-space vortex geometry is fundamentally different from the rib-roll structure in a mixing layer (see e.g. Lin \& Corcos 1984). Hence, any similarity in the $x$-averaged $\omega_{x}$ contours should not be construed to indicate that the underlying vortex dynamics are similar, let alone identical. Additionally, the near-wall educed CS exhibit an inherent overlapping and staggering in $x$, indicating the importance of $x$-dependent $\omega_{x}$ modes, the focus of our study. Finally, Benney focuses on the growth of secondorder perturbations by nonlinear interactions, which is inapplicable to our analysis of linear mechanisms of streak perturbation growth $(\S 4)$. That is, the linear growth we describe can produce eventual nonlinearity, but does not require nonlinearity for growth (unlike the mechanism addressed by Benney).

A related mechanism for vortex generation in near-wall turbulence is proposed by Jang, Benney \& Gran (1986), who apply the 'direct resonance' concept of Benney \& Gustavsson (1981) to the growth of coherent (wave) motion in triple flow decomposition into mean, coherent, and incoherent parts (Reynolds \& Hussain 1972). The direct resonance mechanism consists first of algebraic growth (before eventual exponential decay) of $\omega_{y}$ of a streak due to the vertical velocity $v$ of (stable) oblique modes of the turbulent mean profile - a form of linear transient growth (discussed below). After sufficient growth, the $\omega_{y}$ induced by left- and right-travelling oblique modes then interacts nonlinearly to produce an $x$-averaged $v$, which Jang et al. attribute to streamwise 'vortices'. Although this approach produced some promising results (including a preferred spanwise scale of 90 wall units), its foundation is challenged in Waleffe, Kim \& Hamilton (1993). In particular, the direct resonance growth is in fact much slower than that for many other (non-resonant) modes, and the nonlinear interaction of the oblique modes' $v$ (rather than $\omega_{y}$ ) is actually more dominant, but not considered. Note that physical-space interpretations for such modal 'nonlinear interactions' have so far not been presented.

\subsubsection{Streak instability}

Several studies have targeted 'instability' of lifted low-speed streaks as the dominant agent of turbulence production, although the details of the instability mechanism vary widely in the literature, and the associated physical-space process of vortex generation has not been addressed. This instability concept germinated from early flow visualization studies of low-speed fluid (Kline et al. 1967), which suggested spatial oscillations of local $U(y)$ shear layers of the Kelvin-Helmholtz type, prior to an apparent 'breakdown' into smaller scales.

Based on the evolution of instantaneous structures visualized via DNS, Robinson (1991) proposes that lifted low-speed streaks, left behind by (faster advecting) streamwise vortices, contain a locally unstable $U(y)$ shear on the streak crest which then gives rise to new spanwise 'arch' vortices. One 'leg' of the arch is said to be stretched into a streamwise vortex, which in turn generates a new unstable streak in its wake to close the cycle. Accounting for the entire streak $U(y, z)$ distribution (rather than $U(y)$ only), such a streak-crest $U(y)$ instability mechanism conceptually corresponds to varicose modes, which exhibit a hairpin-type perturbation symmetry. In studies of streak $U(y, z)$ distributions generated by (nonlinear) Görtler vortices (Hall \& Horseman 1991; Yu \& Liu 1991), the growth rates of varicose modes are found to be relatively small (approximately one-half) compared to the dominant sinuous modes (the focus of this paper) - even with exceedingly strong streak-crest $U(y)$ shear. Furthermore, for streak $U(y, z)$ distributions more representative of near-wall turbulence (i.e. with realistic $U(y)$ shear magnitudes), varicose modes are found to 
be stable (see SH). Finally, note that the dominant varicose instability would necessarily be accompanied (in the nonlinear regime) by formation of two-legged hairpin vortices, which are indeed rarely found near the wall. As an alternative explanation of Robinson's observations, we find that new spanwise 'arch' vortices can form due to growth of sinuous disturbances, accompanied by generation of new streamwise vortices (see $\mathrm{SH}$ ). In this scenario, an arch vortex is generated by rollup of an internal shear layer atop a streak, which links up with the downstream end of a nearby streamwise vortex due to the shear-induced collapse of the connecting vortex lines. Owing to circulation pile-up inherent to vorticity layers of finite extent, vortex rollup commences at the tip of the internal shear layer, much like a wing-tip vortex. (Note that no perturbations are required for internal shear layer rollup, and hence the arch formation is not actually an instability process, although vorticity concentration by two-dimensional self-advection is analogous to Kelvin-Helmholtz instability.)

An alternative streak instability mechanism is proposed by Swearingen \& Blackwelder (1987), based on experimental analysis of streak 'breakdown' induced by Görtler vortices, revealing a dominant sinuous (i.e. streak $x$-waviness) mode of transition. From flow visualization and measurements revealing correlation of large fluctuation amplitudes with $z$ inflections of $U$, they also inferred that turbulence production is caused by local, 'wake-like' instability of the $U(z)$ shear layers flanking low-speed streaks. Note the distinction with Kline et al. (1967) and Robinson (1991), who suggested that the instability is driven by Kelvin-Helmholtz-type instability of the streak-crest $U(y)$. Subsequent stability analysis (Yu \& Liu 1991) revealed that Görtler $U(y, z)$ streak distributions, representative of the Swearingen \& Blackwelder experiments, are in fact unstable to (predominant) sinuous modes. The connection of vortex generation in near-wall turbulence with the Görtler-streak breakdown was not addressed in these studies.

As a further extension of the streak instability concept, Hamilton et al. (1995) study vortex regeneration using the 'minimal flow unit' concept of Jimenez \& Moin (1991) applied to plane Couette flow. Hamilton et al. reveal a surprisingly cyclic flow evolution and identify a three-step closed cycle: (i) streak formation by streamwise vortices, (ii) streak 'breakdown' via (normal-mode) sinuous instability, and (iii) 'regeneration' of streamwise vortices due to nonlinear interactions in the post-breakdown flow. While these results are encouraging, particularly from the viewpoint of mechanistic low-order dynamical modelling (Waleffe 1997), the influence of the highly constrained domain on the vortex dynamics underlying regeneration has not been addressed systematically. For instance, the low $R e$ causes ( $x$-averaged) streamwise vortices to completely fill the gap between walls, much like Taylor-Couette 'roll' vortices. Our own analysis indicates fundamental differences in the vortex regeneration dynamics of minimal Couette and channel flows (Schoppa \& Hussain 1998a).

To date, the premise of streak instability has not been established for streaks characteristic of near-wall turbulence, particularly assessment of whether the normal vorticity on streak flanks is sufficiently strong to cause normal-mode instability. Furthermore, the suppression of instability growth by viscous diffusion of (base flow) streak vorticity-potentially significant noting the relatively smallscale characteristic streak spacing-has not been addressed. Finally, a link has not been found between (linear) streak instability and well-documented (finite-amplitude) physical-space structures as well as near-wall turbulence measures. In summary, the role of sinuous normal-mode streak instability in generating the physicalspace CS observed in near-wall turbulence is thus an unresolved issue, addressed here. 


\subsection{Our objectives}

The primary goal of this paper is to explain near-wall vortex generation in fully developed turbulent boundary layers, via growth of $x$-dependent streak perturbations. In particular, we address in detail the potential significance of normal-mode streak instability, through both linear stability analysis and a description of the underlying (three-dimensional) mechanism. Further, the potential for instability of streaks extracted from fully developed turbulence is evaluated. Note that our analysis is based on developed streaks with the documented spanwise spacing of 100 wall units; the now well-known mechanism of streak formation by 'lift-up' is not addressed further here. In response to identified limited growth of normal-mode streak instability, we develop a new scenario-(linear) streak transient growth (STG)-involving (nonnormal) $x$-dependent spanwise velocity perturbations. The underlying mechanism of STG, responsible for its rapid early-time growth, is explained using physical-space vortex dynamics concepts. In particular, physical-space mechanisms are presented to explain: (i) the role of streak shear in generating streamwise vorticity, and (ii) the role of velocity perturbations normal to the streak flank in extracting perturbation kinetic energy from the mean flow. STG amplification into the nonlinear regime is analysed as a new mechanism of streamwise vortex generation, the prevalence of which is established by a close correspondence with documented near-wall turbulence structures: streamwise vortices, internal shear layers, VISA events, $u w$ and $u v$ quadrant Reynolds stresses, and turbulence statistics. Furthermore, detailed cause-and-effect explanations of the generation of streamwise vortices and internal shear layers are pursued using the 'clean' STG-based flow evolution.

In the following, we first outline the computational approach $(\S 2)$, followed by normal-mode stability analysis of near-wall streaks (\$3) and development of an alternative transient growth mechanism $(\S 4)$. The genesis of new streamwise vortices and internal shear layers is illustrated in $\S 5$, along with a detailed description of the vortex dynamics involved and related turbulence statistics. In $\S 6$, key results are summarized, and implications for boundary layer modelling and control are discussed.

\section{Computational approach}

\subsection{Direct numerical simulation}

The linear evolution of streak perturbations and the subsequent nonlinear vortex generation are studied through direct numerical integration of the incompressible Navier-Stokes equations for plane Poiseuille (channel) flow. The fully spectral algorithm of Kim et al. (1987) is used, with periodic boundary conditions in the streamwise $(x)$ and spanwise $(z)$ directions, and the no-slip condition enforced on both walls (normal to $y$ ). Fourier expansion is employed in the homogeneous $(x, z)$ directions, with Chebyshev polynomials in $y$ and cos-mapping for grid concentration near both walls. For time-stepping, the third-order (explicit) Runge-Kutta method is applied to the (nonlinear) advective terms, and the second-order (implicit) Crank-Nicolson scheme is used to advance the viscous terms. For further details of the numerical algorithm, see Kim et al. (1987).

For all simulations, we use the 'minimal channel' domain size of $\left(L_{x}^{+} \approx 300\right.$, $L_{y}^{+} \approx 200, L_{z}^{+} \approx 100$ ) and a bulk Reynolds number of $R e=Q / v=2670$ (where $Q$ is the constant-volume-flow rate per unit $z$ ), for which developed channel flow turbulence is sustained (Jimenez \& Moin 1991). With $32 \times 129 \times 32$ computational points in $(x, y, z)$, grid spacings of $\Delta x^{+}=9.4, \Delta y^{+}$ranging from 0.027 (wall) to 2.3 
(centreline), and $\Delta z^{+}=3.1$ resolve all dynamically significant lengthscales without requiring subgrid-scale modelling.

The (streamwise $(x)$, normal $(y)$, spanwise $(z)$ ) components of velocity and vorticity are denoted as $(u, v, w)$ and $\left(\omega_{x}, \omega_{y}, \omega_{z}\right)$, respectively, with positive and negative values indicated by ' + ' and ' - ' signs. Perturbations of the two-dimensional streak base flow $U(y, z)$ (with time-independent vorticity $\Omega_{y}$ and $\Omega_{z}$ ) are primed $\left(u^{\prime}\right)$, and linear stability (normal-mode) eigenfunctions are tilded $(\tilde{u})$. Turbulent fluctuations of the $(x, z)$-averaged mean profile $U_{0}(y)$ are denoted by $u_{t}$, with r.m.s. amplitude indicated as $u_{r m s}$.

\subsection{Near-wall streaks: problem formulation}

Herein, we isolate the dynamics of three-dimensional perturbations of lifted streaks, in a 'clean' environment free from existing structures and incoherent turbulence (including perturbations induced by larger-scale outer vortices). In particular, we analyse a $z$-periodic row of $x$-independent (i.e. straight and parallel), finite-amplitude low-speed streaks, initially containing no vortices or $\omega_{x}$; hence, the base flow consists of the two-dimensional streak distribution $U(y, z)$ only. This simplification is well justified by the fact that streaks extend in $x$ for lengths an order of magnitude longer than individual near-wall streamwise vortices (see figure 1). Note also that individual streak regions are frequently devoid of significant $\omega_{x}$, consistent with the base flow considered here. The streaks are localized to a single wall, to prevent the second wall (far removed in $z$ ) from strongly influencing the essential near-wall dynamics, as is the case for channel and plane Couette flow at sufficiently high Re. Note that this class of base flows is inviscidly steady for a constant volume flux, and is qualitatively consistent with near-wall streaks observed both in minimal channel flow (Jimenez \& Moin 1991; SH) and fully developed turbulent boundary layers (e.g. Robinson 1991) - both having similar near-wall structures and statistics.

As a representation of vortex-free, lifted low-speed streaks of variable strength, we consider a base flow family of the form

$$
U(y, z)=U_{0}(y)+(\Delta u / 2) \cos \left(\beta_{s} z\right) g(y), \quad V=W=0,
$$

where $U_{0}(y)$ is the mean velocity and $g(y)$ is an amplitude function which satisfies the no-slip condition at $y=0$ and localizes the streak velocity defect to a single wall (i.e. $\left.y^{+}<60\right)$. A function satisfying these requirements is $g(y) \sim y \exp \left(-\eta y^{2}\right)$, normalized to unity and with $\eta$ specified such that the streak velocity defect $\Delta u$ and normal vorticity $\left.\Omega_{y}\right|_{\max }=\beta_{s} \Delta u / 2$ exhibit a plateau in the range $y^{+}=10-30$, consistent with observed lifted streaks and $\omega_{\text {yrms }}$ statistics. Note that the choice of a (single-harmonic) sinusoidal $z$ dependence in (1) is supported well by the streak formation analysis in Waleffe (1995), which demonstrates that the higher $z$ harmonics of the streak $U(y, z)$ distribution are an order of magnitude weaker than the first harmonic.

A 'single-sided' turbulent mean velocity profile is analysed, analogous to that observed in minimal channel turbulence (Jimenez \& Moin 1991), with a parabolic profile $U_{\text {lam }}$ in the laminar top half of the channel and a Reichardt profile $U_{\text {turb }}$ in the turbulent bottom half:

$$
U_{0}(y)=\left\{\begin{array}{l}
U_{\text {lam }}=U_{c}\left[1-((y / h)-1)^{2}\right], \quad y_{m} \leqslant y \leqslant 2 h \\
U_{\text {turb }}=u_{*}\left[2.5 \ln (1+0.4 y / \delta)+7.8\left(1-\mathrm{e}^{(-y / 11 \delta)}-\frac{y}{11 \delta} \mathrm{e}^{-0.33 y / \delta}\right)\right], \\
\quad 0 \leqslant y<y_{m}
\end{array}\right.
$$

Note that the turbulent mean profile $U_{\text {turb }}$ is used as a first approximation of the local 


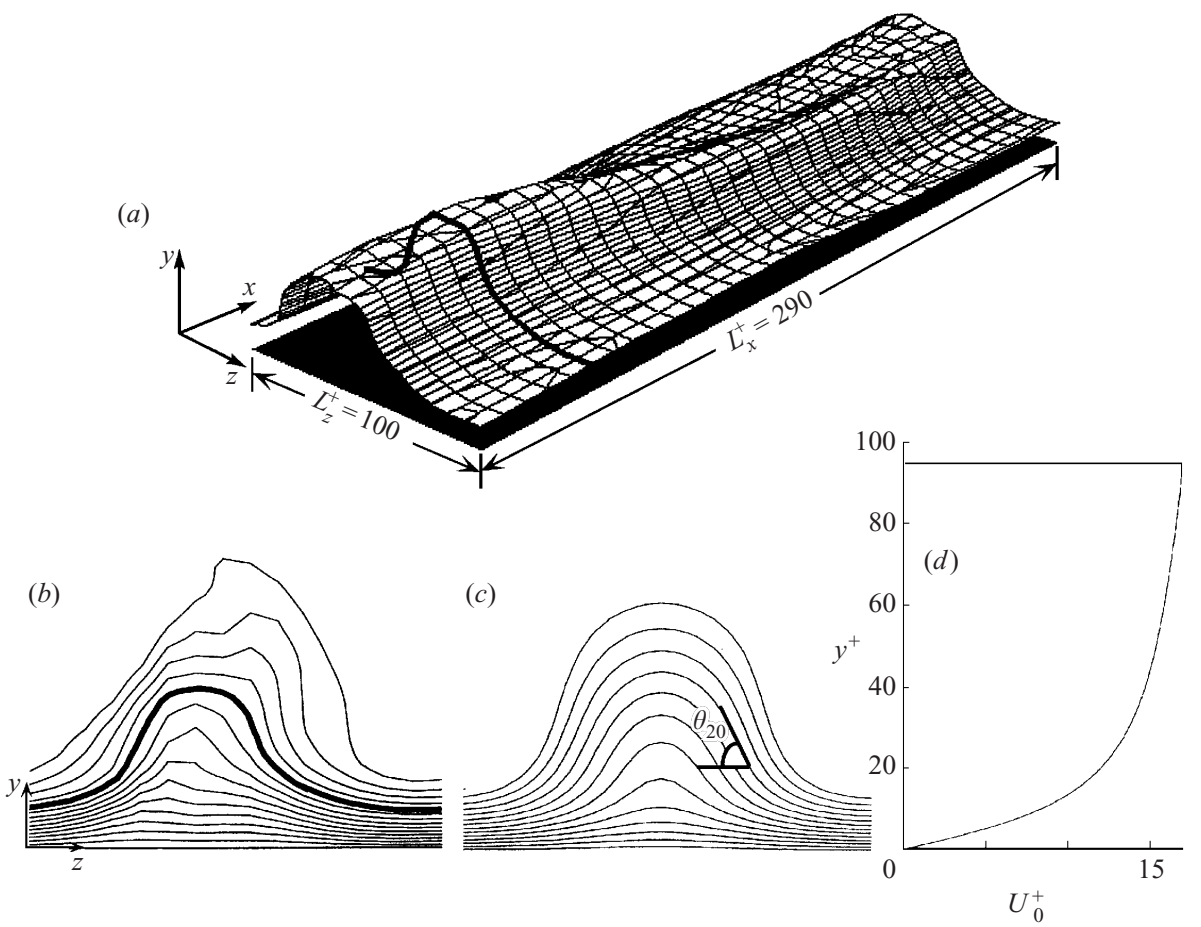

FIGURE 2. Low-speed streak realization in minimal channel flow: $(a) 0.55 U_{c}$ isosurface, $(b)$ typical $U(y, z)$ cross-stream distribution, with the bold contour common to $(a)$ and $(b)$. Streak base flow used for stability analysis: (c) $U(y, z)$ distribution (1) with $\theta_{20}=56^{\circ}$ (with $\theta_{20}$ definition indicated at $\left.y^{+}=20\right) ;(d)$ mean velocity $U_{0}(y)$ profile (2) for one-sided turbulent channel flow (channel centreline at $y^{+}=95$ ).

mean flow $U_{0}(y)$ of streaks observed in near-wall turbulence, and is held constant to isolate the effects of spanwise shear across streaks from the mean shear. The two profiles $U_{\text {turb }}$ and $U_{\text {lam }}$ are matched at a location $y_{m}$ in the turbulent half, with $y_{m}$ and $U_{c}$ determined so that the mean flow velocity and vorticity are continuous at the matching location, i.e.

$$
U_{\text {lam }}\left(y_{m}\right)=U_{\text {turb }}\left(y_{m}\right) \quad \frac{\mathrm{d} U_{\text {lam }}}{\mathrm{d} y}\left(y_{m}\right)=\frac{\mathrm{d} U_{\text {turb }}}{\mathrm{d} y}\left(y_{m}\right) .
$$

Furthermore, the friction velocity $u_{*}$ and viscous (wall) lengthscale $\delta$ in (2) are chosen in accordance with Dean's (empirical) correlation for fully developed turbulent channel flow:

$$
u_{*}=\frac{Q}{2 h} \sqrt{0.0365(Q / v)^{-0.25}}, \quad \delta=v / u_{*},
$$

reflecting the physical constraint that the friction velocity cannot be chosen independently of the volume flow rate and Reynolds number. For all flows considered here (with $R e=Q / v=2670$ ), the matching conditions (3) are satisfied by $y_{m}=0.92 h$ and $U_{c}=1.2 Q /(2 h)$, resulting in the mean velocity profile $U_{0}(y)$ illustrated in figure $2(d)$.

As illustrated in figure $2(c)$ for a moderately strong streak (with the streak-flank vorticity $\omega_{y}$ governed by $\Delta u$ in (1)), the base flow (1)-(2) closely resembles the straight and $x$-uniform low-speed streak regions observed at the quiescent phase (i.e. time of minimum drag) of minimal channel flow (figure $2 a, b$ ). In accordance with (1), all streak base flows considered here are even-symmetric about $z=0$, i.e. $U(y, z)=U(y,-z)$. 
Note that the streaks are localized to a single wall (via $g(y)$ in (1)), and hence are essentially decoupled from the second wall (far removed in $y$ ). Thus, this class of base flows does not exhibit any $y$ symmetries, unlike prior instability studies of streaks bounded closely by both walls in low-Re plane Couette and channel flow. Compared to single-walled streaks, the influence of a second no-slip wall immediately above the streak is twofold: (i) additional $y$ symmetry is imposed on the linear eigenmodes and (ii) the subsequent nonlinear evolution is fundamentally altered (discussed in Schoppa \& Hussain 1998a). As an example, thin sheets of $\omega_{x}$ (reflecting local jet-like $w(y)$ flow) are generated in narrow-gap Couette flow, compared to collapsed streamwise vortices generated from single-walled streaks in channel flow.

For illustrative purposes, it is useful to represent the 'strength' of lifted streaks (i.e. the magnitude of streak wall-normal vorticity $\Omega_{y}=\partial U / \partial z$ ) in terms of the base flow vortex line inclination angle $\theta$ on the streak flank, given locally by $\theta=\tan ^{-1}\left(\left|\Omega_{y}\right| /\left|\Omega_{z}\right|\right)$. For the base flow streaks (1), the characteristic vortex line lift angle is defined at $y^{+}=20$ as $\theta_{20}=\tan ^{-1}\left[\left.\Omega_{y}\right|_{\max } /\left(\mathrm{d} U_{0} / \mathrm{d} y\right)\right]_{y^{+}=20}$, where $\left.\Omega_{y}\right|_{\max }=\beta_{s} \Delta u / 2$ and $\mathrm{d} U_{0} / \mathrm{d} y$ is the mean flow spanwise vorticity, e.g. $\theta_{20}=56^{\circ}$ for the streak in figure $2(c)$.

Note that the amplitude function $g(y)$ in (1) determines the strength of the local streak-top $U(y)$ shear layer (e.g. local maximum of $\partial U / \partial y$ ) residing on the crest of the lifted streak. Instability growth rates for the predominant sinuous modes (with symmetry defined below) - the focus of this study-are found to be relatively insensitive to the strength of this shear layer and hence to the amplitude function $g(y)$. Note, however, that the slower-growing varicose mode is found to depend crucially on the vorticity magnitude of this wall-detached (free) shear layer. Varicose mode instability is possible for artificially strong streak-top shear, yet its growth rate is significantly smaller than the corresponding sinuous mode (e.g. Görtler streaks, see Yu \& Liu 1991). Thus, the $y$-variation of the streak velocity defect (represented here by $g(y)$ ) should be varied systematically in instability studies of varicose modes. Varicose modes are found to be stable for the streaks considered here, representative of near-wall turbulence $(\mathrm{SH})$, and are not addressed further.

For all flows considered here, the streak spanwise wavenumber $\beta_{s}$ in (1) is chosen as $2 \pi / \beta_{s}^{+}=100$, corresponding to the well-accepted average spanwise spacing of adjacent low-speed streaks noted in numerous experimental and numerical studies. As discussed below, the existence of a predominant streak spanwise spacing is consistent with two competing mechanisms: (i) enhanced perturbation growth for smaller streak spacings due to streaks' more intense wall-normal vorticity $\Omega_{y}$, and (ii) reduced streak diffusion at larger streak spacings (with weakened $\Omega_{y}$ ), which sustains perturbation growth for a longer period of time. Although our analysis may subsequently be extended to address the predominance of this particular streak spacing (via parametric analysis of $\beta_{s}$ ), our focus here is on vortex generation from developed streaks, whose spacing must thus be specified a priori. Note that the complementary mechanism of streak formation, i.e. lift-up of low-speed fluid near the wall by the induced $v$ of (mature) streamwise vortices, is well-documented (see Waleffe 1995 for details).

\subsection{Stability analysis approach}

Due to the two-dimensionality of the base flow (1), direct solution of the associated two-dimensional p.d.e. eigenvalue problem (defined below) necessitates a complex computational algorithm such as spectral collocation (with grid mapping in $y$ to resolve the single-walled streaks), involving eigensolution of large, non-sparse matrices. As an alternative, we analyse the instability of the streak base flow (1) using direct numerical simulations (DNS) of the Navier-Stokes equations, initialized with infinites- 
imal disturbances. For the finite-Re stability analysis pursued here, the $x$-independent modes constituting the base flow are 'frozen', i.e. the streak is held constant in time.

This DNS-based approach is well-suited to extract most-unstable (or least-stable) linear normal modes. That is, individual modes of interest may be analysed through appropriate choices of small-amplitude disturbances, including specification of the streamwise and spanwise wavenumbers $(\alpha, \beta)$ and a sinuous spanwise symmetry. Provided that such an arbitrary perturbation has a non-zero projection onto the instability mode of interest, the perturbation evolution will naturally evolve to the most unstable (or least-stable) eigenmode. 'Lock-on' of the simulation to a given linear normal mode is signalled by sustained exponential growth (or decay) of $E_{3 D}(t)$, the volume-integrated energy in all $x$-dependent Fourier modes. Furthermore, non-normal linear disturbances resulting in 'transient growth'-outside the scope of traditional normal mode stability analysis - may be analyzed through an appropriate initial value problem (discussed in $\S 4$ ). As validation of both this stability analysis approach and the DNS code, arbitrary linear-amplitude two- and three-dimensional disturbances were verified to lock on to the corresponding Orr-Sommerfeld mode (Orszag 1971), with growth rate agreement within $1 \%$.

\section{Linear normal-mode instability of streaks}

To evaluate the role of streak instability in vortex generation, we analyse ( $\$ 3.1$, 3.2) the three-dimensional instability of the two-dimensional streaks (1), over a range of streak strengths (i.e. magnitude of the streak-flank vorticity $\Omega_{y}$ ), represented by the streak lift angle $\theta_{20}$. Our focus here is on 'lifted' streaks, which are detectable even outside the buffer layer (e.g. at $y^{+}=30$; see Robinson 1991). Note the distinction of these lifted streaks from the more numerous sublayer streaks, which are localized to the viscous sublayer but do not extend into the buffer layer, reflecting the variation of streak count with $y$ observed by Adrian et al. (1999). (A lifted streak is typically traceable to a particular sublayer streak, but the inverse is not always true.) We then evaluate the instability results with respect to streaks extracted from fully developed turbulence (\$3.3), indicating that only a small fraction of streaks are sufficiently strong for instability. The inherently three-dimensional streak instability perturbation is compared with other free shear flow instabilities in $\S 3.4$, revealing a close connection with a new type of corrugated mixing-layer instability. The role of viscous effects, particularly instability suppression by the streaks' viscous selfannihilation, is described in $\S 3.5$.

\subsection{Stability problem formulation}

In accordance with Floquet theory for the $z$-periodic base flows represented in (1), we consider temporal disturbances (denoted by primes) of the form

$$
\left(\begin{array}{c}
u^{\prime} \\
v^{\prime} \\
w^{\prime} \\
p^{\prime}
\end{array}\right)(x, y, z, t)=\operatorname{Re}\left[\left(\begin{array}{c}
\tilde{u} \\
\tilde{v} \\
\tilde{w} \\
\tilde{p}
\end{array}\right)(y, z) \mathrm{e}^{\mathrm{i}(\alpha x+\beta z)} \mathrm{e}^{\sigma t}\right],
$$

where the streamwise $(\alpha)$ and spanwise $(\beta)$ wavenumbers are real, with eigenvalues $\sigma=\sigma_{r}+\mathrm{i} \sigma_{i} ;$ Re denotes the real part. The tilded (complex) eigenfunctions are periodic in $z$ with the streak spanwise wavenumber $\beta_{s}$, and the velocity eigenfunctions vanish at the bottom and top walls $(y=0,2 h)$.

Linearizing the Navier-Stokes equations and substituting (5) for perturbations, one 
obtains the eigenproblem

$$
\begin{gathered}
\sigma \tilde{u}+U i \alpha \tilde{u}+\tilde{v} \frac{\partial U}{\partial y}+\tilde{w} \frac{\partial U}{\partial z}=-\mathrm{i} \alpha \tilde{p}+\frac{1}{\operatorname{Re}} \tilde{\nabla}^{2} \tilde{u} \\
\sigma \tilde{v}+U \mathrm{i} \alpha \tilde{v}=-\frac{\partial \tilde{p}}{\partial y}+\frac{1}{\operatorname{Re}} \tilde{\nabla}^{2} \tilde{v} \\
\sigma \tilde{w}+U \mathrm{i} \alpha \tilde{w}=-\mathrm{i} \beta \tilde{p}-\frac{\partial \tilde{p}}{\partial z}+\frac{1}{\operatorname{Re}} \tilde{\nabla}^{2} \tilde{w} \\
\mathrm{i} \alpha \tilde{u}+\frac{\partial \tilde{v}}{\partial y}+\mathrm{i} \beta \tilde{w}+\frac{\partial \tilde{w}}{\partial z}=0 \\
\tilde{\nabla}^{2} f \equiv-\alpha^{2} f+\frac{\partial^{2} f}{\partial y^{2}}-\beta^{2} f+2 \mathrm{i} \beta \frac{\partial f}{\partial z}+\frac{\partial^{2} f}{\partial z^{2}}
\end{gathered}
$$

with the boundary conditions given above. In (6), the wavenumbers $(\alpha, \beta)$ and $R e$ are fixed parameters for a given mode, and the real and imaginary parts of the eigenvalue $\sigma$ represent the (temporal) growth rate $\left(\sigma_{r}\right)$ and phase speed $\left(\sigma_{i} / \alpha\right)$, respectively, of the corresponding eigenfunction.

Eigenmode symmetries. Several important structural features of streak instability modes, which constrain the permissible physical-space perturbation distributions, are revealed by examining the symmetry properties of the governing equations. Due to the $x$-homogeneity and even $z$-symmetry of the base flow (1), the eigenfunction equations (6) exhibit several symmetries, described as follows. First, note that by taking the complex conjugate $\left(^{*}\right)$ of $(6)$, an eigenfunction for $(-\alpha,-\beta)$ with eigenvalue $\sigma^{*}$ can be constructed from one belonging to $(\alpha, \beta)$ and $\sigma$, i.e.

$$
\left(\begin{array}{l}
\tilde{u} \\
\tilde{v} \\
\tilde{w} \\
\tilde{p}
\end{array}\right)(\alpha, \beta, \sigma) \rightarrow\left(\begin{array}{l}
\tilde{u}^{*} \\
\tilde{v}^{*} \\
\tilde{w}^{*} \\
\tilde{p}^{*}
\end{array}\right)\left(-\alpha,-\beta, \sigma^{*}\right) .
$$

Similarly, an eigenfunction for $-\beta$ and $\sigma$ can be obtained upon reflection about $z=0$ and sign reversal of the spanwise velocity component:

$$
\left(\begin{array}{l}
\tilde{u}(y, z) \\
\tilde{v}(y, z) \\
\tilde{w}(y, z) \\
\tilde{p}(y, z)
\end{array}\right)(\alpha, \beta, \sigma) \rightarrow\left(\begin{array}{l}
\tilde{u}(y,-z) \\
\tilde{v}(y,-z) \\
-\tilde{w}(y,-z) \\
\tilde{p}(y,-z)
\end{array}\right)(\alpha,-\beta, \sigma),
$$

indicating, along with successive application of (7) and (8), that only positive values of the parameters $\alpha$ and $\beta$ need to be considered in stability analysis. More importantly, (8) reflects a structural distinction with oblique instability modes of a parallel shear layer, for which modes for $+\beta$ and $-\beta$ differ only in the sign of $\tilde{w}$ and simply represent left- or right-propagating spanwise waves (Schoppa, Hussain \& Metcalfe 1995). In contrast, streak instability modes for $+\beta$ and $-\beta$ have eigenfunction $z$ distributions (i.e. related via (8) by spanwise reflection about $z=0$ ), which preclude a spanwise-wave-type structure (discussed below).

A further instructive symmetry occurs for inviscid disturbance evolution, in which the viscous terms of (6) are omitted. In this special case, an eigenfunction for $-\sigma^{*}$ 
(a)

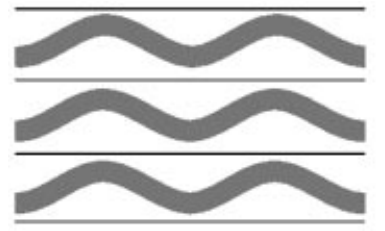

$(d)$

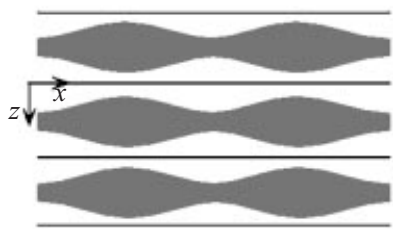

(b)

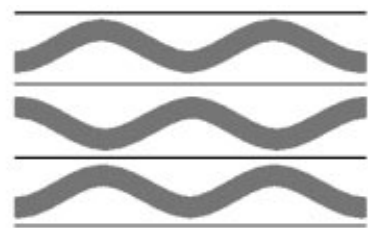

(e) (c)

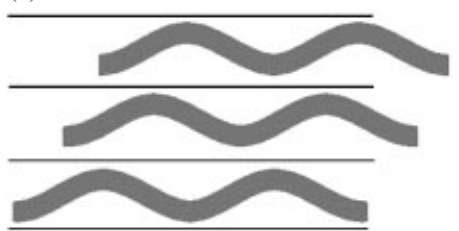

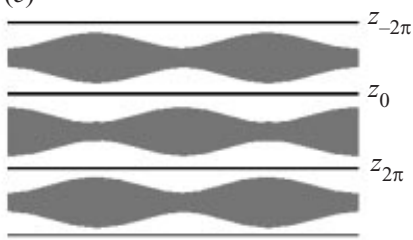

FigURE 3. Illustration of linear instability perturbation effect on base-flow streaks (grey-shaded) for modes: $(a)$ fundamental sinuous, $(b)$ subharmonic sinuous, $(d)$ fundamental varicose, and $(e)$ subharmonic varicose. A travelling spanwise wave as in $(c)$ is not permissible as a streak instability mode, due to $z$-symmetry constraints.

and $-\beta$ can be generated from one belonging to $\sigma$ and $\beta$ as

$$
\left(\begin{array}{l}
\tilde{u} \\
\tilde{v} \\
\tilde{w} \\
\tilde{p}
\end{array}\right)(\alpha, \beta, \sigma) \rightarrow\left(\begin{array}{l}
-\tilde{u}^{*} \\
\tilde{v}^{*} \\
\tilde{w}^{*} \\
-\tilde{p}^{*}
\end{array}\right)\left(\alpha,-\beta,-\sigma^{*}\right) .
$$

That is, for every stable (decaying) inviscid mode $\left(\sigma_{r}<0\right)$, there exists a corresponding unstable $\left(\sigma_{r}>0\right)$ mode (the latter being constructed by applying (9) and (8) successively), indicating that the base flow is either unstable or neutrally stable $\left(\sigma_{r}=0\right)$ to inviscid disturbances. That is, the base flow cannot have decaying inviscid modes only; the existence of only decaying modes thus requires viscous effects.

Sinuous and varicose modes. To consider the physical space perturbation effect of streak instability modes, we consider the relevant $z$-symmetries. Note that these symmetries are apparent $a$ priori if $z=0$ is aligned with the lines in figure 3 . For the case of $z$-fundamental modes (i.e. $\beta=0$, no spanwise propagation), it is well-known that for $z$-symmetric base flows, the eigenfunctions of (6) split into two classes: (i) 'varicose' modes (figure $3 d$ ) with $(\tilde{u}, \tilde{v}, \tilde{w})$ : (even, even, odd) in $z$, and (ii) 'sinuous' modes (figure $3 a$ ) with $(\tilde{u}, \tilde{v}, \tilde{w})$ : (odd, odd, even) in $z$.

Note that a similar $z$-symmetry is present in the governing equations for normalmode perturbations $\left(u^{\prime}, v^{\prime}, w^{\prime}\right)$, resulting in two distinct perturbation classes with even and odd $z$-symmetries. (This can be easily verified by: (i) decomposition of perturbations into $z$-even and $z$-odd components, (ii) integration of the linearized Navier-Stokes equations (NSE) over an arbitrary interval $\left[-z_{0},+z_{0}\right]$ to obtain the even perturbation equations (odd terms vanish identically), and (iii) subtraction of the even equations from the NSE to yield the odd perturbation equations.) Note that $z$-subharmonic streak instability modes occur as either sinuous (figure $3 b$ ) or varicose (figures $3 e$ ) perturbations. (The terms 'varicose' and 'sinuous' applied to $z$-subharmonic modes refer to the shape of an individual perturbed streak.) When the sign of $\beta$ is switched, the patterns in figure $3(b, e)$ are shifted in $z$ by $2 \pi$, serving as a visual interpretation of the symmetry (8). In contrast, a travelling spanwise wave (figure $3 c$ ), analogous to a single oblique mode of a parallel free shear layer, is not re- 

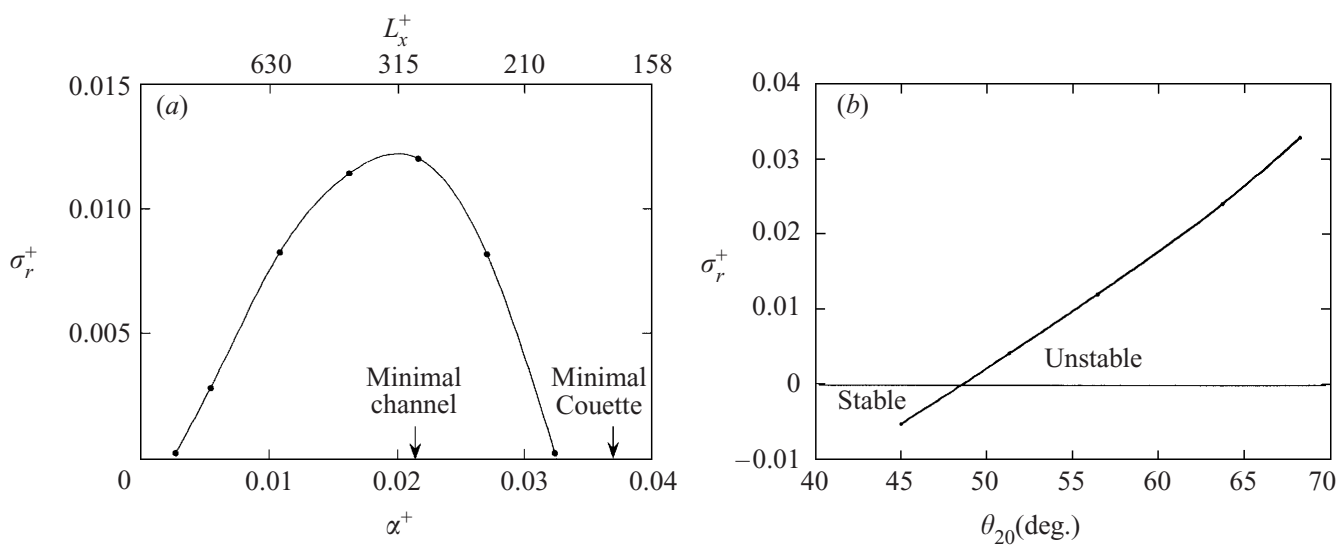

FIGURE 4. Growth rates of unstable normal modes of sinuous streak instability: $(a)$ as a function of streamwise wavenumber $\alpha$, for a streak lift angle of $\theta_{20}=56^{\circ} ;(b)$ as a function of streak streangth $\theta_{20}$ for the most unstable mode with $\alpha^{+}=0.02$.

alizable for streak instability due to the $z$-symmetry described. Hence, theories which invoke oblique modes of a one-dimensional shear layer, without explicit inclusion of $z$-dependence, permit travelling oblique modes analogous to figure $3(c)$, though not permitted for streaks because of the even or odd $z$-symmetry required. Collectively, the symmetry conditions outlined above constrain the permissible physical-space geometry of velocity field perturbations for individual streak instability modes, guiding a priori the functional form of eigenmode solutions. In the following, we focus exclusively on the (dominant) fundamental sinuous mode (figure $3 a$ ).

\subsection{Streak instability results}

To quantify possible linear instability of streaks occurring in near-wall turbulence, we first discuss (normal-mode) solutions of the stability equations (6) for the $U(y, z)$ base flow (1), over a range of streak strengths (i.e. varying $\theta_{20}$ ). Statistics of $\theta_{20}$ are then obtained for streaks extracted from near-wall turbulence, permitting assessment of these streaks' possible instability. All results reported in this section are for $R e=2670$ with the base flow $U(y, z)$ frozen, to isolate viscous effects on the perturbation evolution (viscous influence on the base flow is addressed in §3.5). Note that our prior analysis $(\mathrm{SH})$ of streak instability at higher $R e$ indicates an inviscid instability mechanism -in spite of close proximity to the wall-with increasing growth rate as $R e$ is increased up to an asymptotic limit (for a fixed base flow $U(y, z)$ ).

Figure $4(a)$ shows the normal-mode growth rate $\sigma_{r}^{+} \equiv \sigma_{r} v / u_{*}^{2}$ as a function of streamwise wavenumber $\alpha^{+} \equiv \alpha v / u_{*}$, with the corresponding $x$-wavelength $L_{x}^{+}$denoted on the top axis. The dependence of $\sigma_{r}^{+}$on the streak strength, namely $\theta_{20}$, is shown in figure $4(b)$. As indicated in figure $4(a)$, a moderately strong streak, say with lift angle $\theta_{20}=56^{\circ}\left(\left.\Omega_{y}^{+}\right|_{\max }=0.35\right.$ ) (figure $\left.2 c\right)$ and $2 \pi / \beta_{s}^{+}=100$, is indeed linearly unstable, with a maximum growth rate of approximately $\sigma_{r}^{+}=0.012$. Interestingly, the maximal normal mode growth rate occurs for a streamwise wavelength of approximately 300 wall units, closely corresponding to the minimum $x$-wavelength $\left(L_{x}^{+}=290\right)$ required for turbulence sustenance (Jimenez \& Moin 1991) at $R e=2670$. Note that the 320 wall unit streamwise extent of two $x$-displaced counter-rotating adjacent coherent structures educed from near-wall turbulence (Jeong et al. 1997) also exhibits a nearly maximal streak instability growth rate. Collectively, these results indicate that the characteristic streamwise wavelength of near-wall structures $(\sim 300$ 


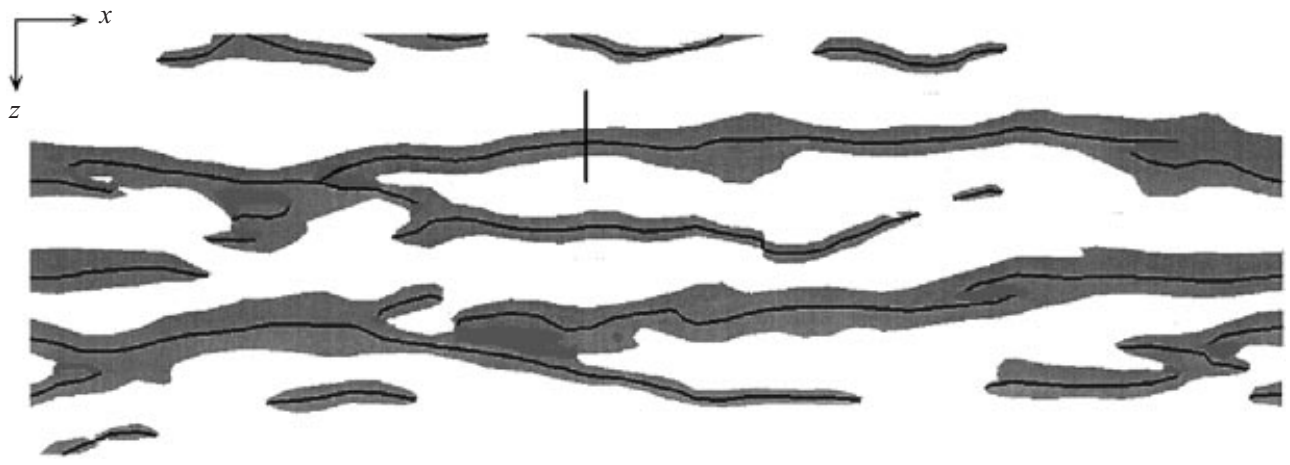

FIGURE 5. Streak identification shown for a typical flow field: streaks at $y^{+}=20$ identified as regions of $u^{\prime}<0$ (grey-shading), with streak centres determined by the local $z$-minimum of $|\partial u / \partial z|$ within each streak region (bold lines).

wall units) is consistent with these streak instability results. As a further note, the minimal $x$-wavelength for sustained turbulent plane Couette flow (Hamilton et al. 1995) - approximately 170 wall units - differs markedly from that of minimal channel flow (figure $4 a$ ), suggesting inherent differences between the two cases.

Having shown linear instability of a $U(y, z)$ distribution visually representative of instantaneous lifted streaks in near-wall turbulence (cf. figure $2 b, c$ ), we now quantify the growth rate variation with streak strength, denoted by the streak lift angle $\theta_{20}$ (defined above). Recall that for a fixed streak spacing, $\theta_{20}$ determines both the height and the flank-slope of lifted $U$ contours (figure $2 c$ ). Results below are for instability modes with a fixed streamwise wavelength of $L_{x}^{+}=300$, approximately the most unstable mode for this range of $\theta_{20}$. Significantly, as shown in figure $4(b)$, sinuous streak instability requires a threshold streak lift angle of approximately $\theta_{20}=50^{\circ}$ (corresponding to a streak vorticity of $\left.\Omega_{y}^{+}\right|_{\max }=0.27$ ). Thus, lifted streaks may be either passive (stable) or dynamically active (unstable) to small-amplitude sinuous perturbations, depending upon rather slight (i.e. visually indistinguishable) differences in streak-flank vorticity. For instance, streaks with a lift angle of $45^{\circ}$ are well within the stability region, while $55^{\circ}$ streaks (which visually look similar) exhibit a significant instability growth rate. Furthermore, this instability threshold indicates that lifted streaks, even those extending past the buffer layer, are not necessarily unstable, depending on the streak-flank slope. On increasing $\theta_{20}$ above the instability cutoff, the growth rate increases approximately linearly with the streak-flank vorticity $\left.\Omega_{y}\right|_{\max }=\partial U / \partial z$, suggesting a dominant influence of $U(z)$ spanwise shear in driving sinuous instability (see also Yu \& Liu 1991 for Görtler streaks). Nevertheless, as shown below (\$3.4), the sinuous mode is inherently three-dimensional, and its growth mechanism is fundamentally different from a one-dimensional $U(z)$ wake profile.

\subsection{Streak strength statistics}

Owing to the growth rate threshold in figure $4(b)$, the role of (normal-mode) streak instability in fully developed near-wall turbulence relies critically on the magnitude of streak lift angle $\theta_{20}$ (hence $\partial u / \partial z$ ) actually realized. To obtain conditional streak statistics, individual streak realizations are extracted from fully developed turbulent channel flow at $R e=Q / v \sim 3100$ (Kim et al. 1987 database). To obtain statistics of 


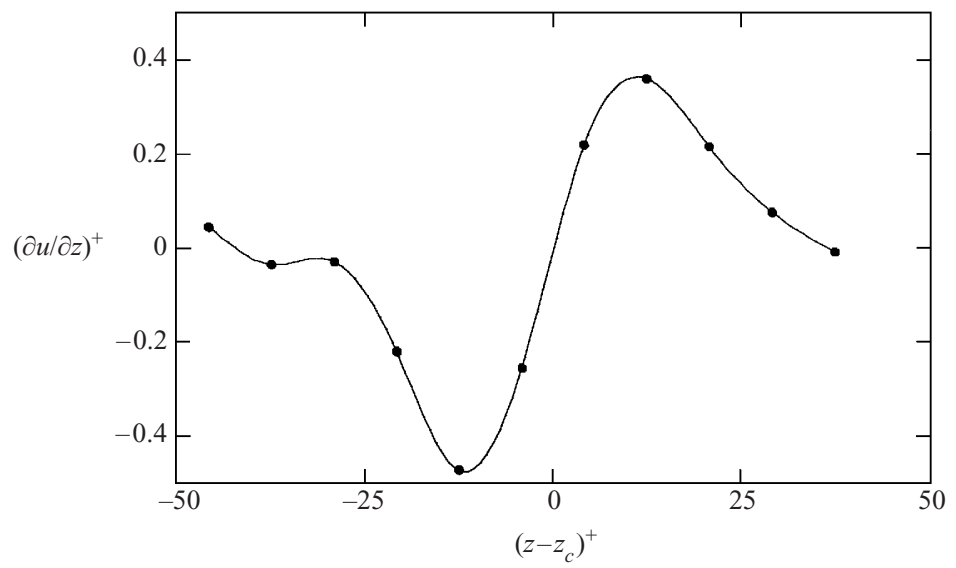

FIGURE 6. A sample local spanwise profile of $(\partial u / \partial z)^{+}$through the streak identified by the vertical line in figure 5; the first local extrema on either side of the streak centre $z_{c}$ defines the streak-flank vorticity.

local, unsmeared streak vorticity, the following streak extraction procedure (illustrated in figures 5 and 6 ) is used:

(i) regions of $u^{\prime}<0$ are identified in a specified $y$-plane (grey-shaded in figure 5);

(ii) within each $u^{\prime}<0$ region, the $\left(x_{c}, z_{c}\right)$ locations of local minima of $u^{\prime}$ in $z$ are identified as streak centres (bold lines in figure 5);

(iii) the first local maxima of $|\partial u / \partial z|$ in $z$ (figure 6) is identified on either side of each streak centre $\left(x_{c}, z_{c}\right)$. The larger of these two $|\partial u / \partial z|$ values is recorded as the streak-flank vorticity $|\partial u / \partial z|_{\max }$ for each streak realization.

Note that the positive and negative peaks of $\partial u / \partial z$ are generally of comparable magnitude (figure 6), reminiscent of a local $U(z)$ wake profile. For flow realizations extracted at 50 different times (spanning 500 wall time units) from fully developed near-wall turbulence $\left(L_{x}^{+} \sim 1400 ; L_{z}^{+} \sim 450\right)$, this extraction procedure identifies approximately 11300 streak $(y, z)$ cross-sections (like the example realization in figure 6). Dividing the $z$ domain size by the average number of streak realizations per unit $x$ (for an $x$ grid spacing of $\Delta x^{+}=29$ ), an average spanwise spacing (between accepted realizations) of 96 wall units is obtained at $y^{+}=20$. The close agreement of this sampled streak count with the accepted $z$-spacing of streaks (100 wall units) confirms that streaks are accurately captured (i.e. false streak counts or omissions are minimal).

Subject to the conditional streak sampling outlined above, histograms of streak lift angle statistics for fully developed turbulence are shown in figure 7 at heights of $y^{+}=$ 10,20 , and 30 from the wall. Analogous to the definition of $\theta_{20}$ above, the characteristic streak lift angle at a given $y^{+}$is defined as $\theta_{y+}=\tan ^{-1}\left[|\partial u / \partial z|_{\max } /\left(\mathrm{d} U_{0} / \mathrm{d} y\right)\right]_{y+=n}$. Note that $\theta_{y+}$ serves only as a characteristic streak strength (for statistical purposes), as the vortex line lift angle of an instantaneous streak can differ from its $\theta_{y+}$ value, owing to: (i) use of only the $\partial u / \partial z$ contribution to $\omega_{y}$ and (ii) non-dimensionalization by the mean shear $\mathrm{d} U_{0} / \mathrm{d} y$, instead of the local $\omega_{z}$. Histograms of streak lift angle $\theta_{y+}$ in figure 7 indicate that larger $\theta_{y+}$ values are more common with increasing $y^{+}$. In particular, the mean lift angles of the streak realizations identified at $y^{+}=10$, 20 , and 30 are $\theta_{10}=25^{\circ}, \theta_{20}=44^{\circ}$, and $\theta_{30}=55^{\circ}$, respectively. The occurrence of stronger streaks at larger $y$ reflects the characteristic geometry of streak vortex lines 


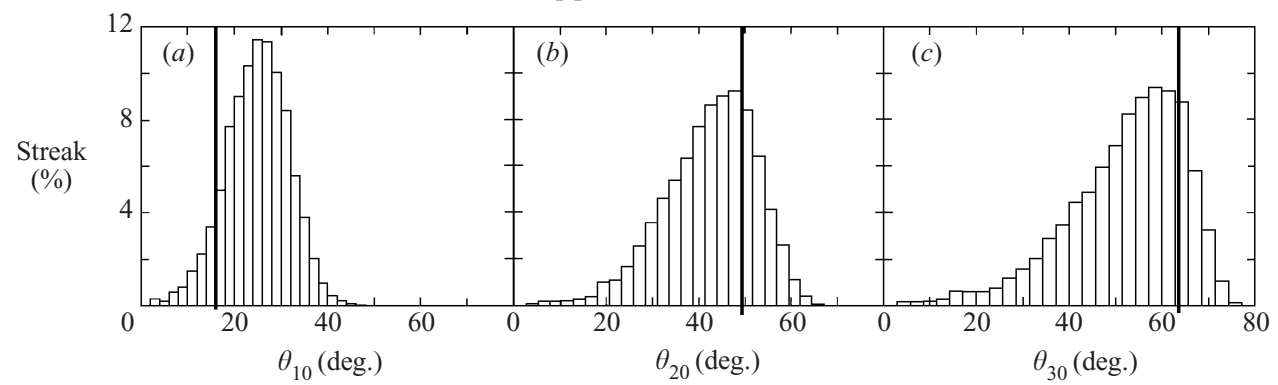

FIGURE 7. Histograms of streak lift angle $\theta_{y+}$, conditionally sampled from DNS data of fully devoloped channel flow turbulence at: $(a) y^{+}=10,(b) y^{+}=20$, and $(c) y^{+}=30$. The bold line at each $y^{+}$denotes the threshold streak lift angle required for instability of the $U(y, z)$ distribution (1).

(figure $2 b, c$ ): nearly flat vortex lines in the sublayer, with strong lifting appeared only above $y^{+} \sim 20$. Note also that only lifted streaks are sufficiently strong to extend into the buffer layer, so that the percentage of strong streaks necessarily increases with $y$ (despite the decreasing total streak count with $y$ due to 'drop-out' of weaker sublayer streaks).

To evaluate whether streaks strong enough for instability are actually realized in near-wall turbulence, the bold lines in figure 7 denote the values of $\theta_{10}, \theta_{20}$ and $\theta_{30}$ for the neutrally stable streak in figure $4(b)$, i.e. the streak $U(y, z)$ distribution (1) with $\theta_{20}=50^{\circ}$. Caution must be exercised in interpreting these results, owing to differences in vorticity distributions between individual streak realizations (extracted from fully turbulent flow) and the base flow (1). In particular, instability should not be inferred for: (i) streaks which may exceed the strength threshold at some $y$-locations (e.g. in the sublayer), but not at all other $y$, and (ii) streaks which are locally strong, but not sufficiently elongated in $x$ (to permit growth of disturbances of wavelength $L_{x}^{+} \sim 300$ ). That is, the presence of streak realizations with $\theta_{y+}$ exceeding the strength threshold for instability serves as a necessary, but not sufficient, condition for the operation of (normal-mode) streak instability in near-wall turbulence. Furthermore, owing to the prominence of weaker (hence stable) sublayer streaks (i.e. not extending into the buffer layer) in statistics for $y^{+} \leqslant 10$, instability should be inferred from statistics in the buffer layer (where only lifted streaks occur). In the range $y^{+}=20-30$, comparison of lift angle statistics (figure $7 b, c$ ) with the corresponding streak instability cutoff (marked by a bold line) indicates that $15-25 \%$ of lifted streaks are strong enough (i.e. with sufficient magnitudes of $\partial u / \partial z$ or $\theta_{20}$ ) to be linearly unstable.

In summary, streaks satisfying the necessary strength condition for linear instability do in fact occur in near-wall turbulence over the range $y^{+}=10-30$, albeit constituting only a small fraction of all lifted streak regions in the buffer region. Additionally, the streak count declines sharply with increasing $\theta_{20}$ near the stability cutoff (e.g. figure $7 b$ ), indicating that few unstable streaks attain a large growth rate (cf. figure $4 b, 7 b)$. Finally, note that other possible mitigating factors of streak instability, particularly the influence of viscous self-annihilation of base flow streak vorticity, must also be considered ( $\S 3.5)$. Hence, a scenario of dominant vortex generation and turbulence sustenance via linear (normal-mode) instability of lifted near-wall streaks must be evaluated carefully, as done below.

\subsection{Relation with other shear-layer instabilities}

Having demonstrated that sufficiently strong near-wall streaks are linearly unstable, we now address the physical mechanism of (normal-mode) streak instability, in re- 
(a)

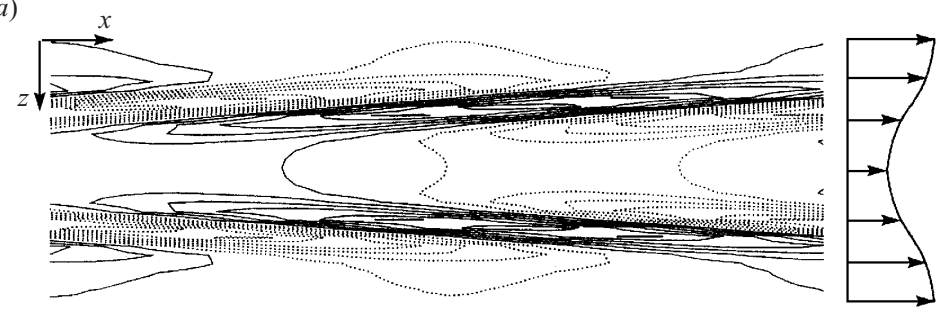

(b)

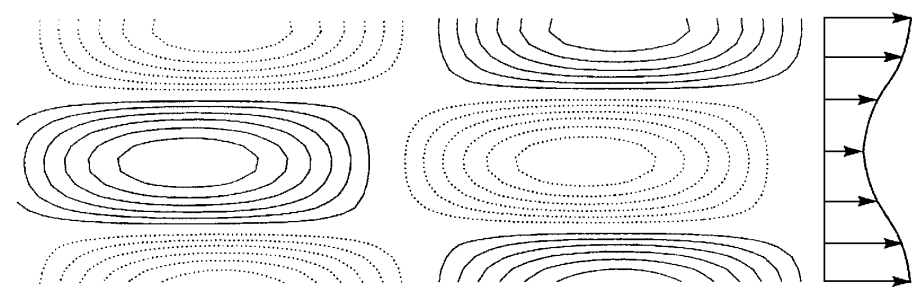

Figure 8. Comparison of $\omega_{y}^{\prime}(x, z)$ distributions of sinuous instability modes of $(a)$ near-wall streaks at $y^{+}=25$ and $(b)$ a one-dimensional sinusoidal wake with the same $U(z)$ profile in this plane.

lation to free shear flow instabilities. Note that the instability is indeed inviscid (i.e. increasing growth rate with $R e$ to an asymptotic limit) in spite of its location in the highly viscous near-wall region. Many prior studies have addressed possible mechanisms of streak instability via localized velocity profile inflections - either inflectional $U(y)$ at the low-speed streak crest (Kelvin-Helmholtz instability, e.g. Kline et al. 1967) or an inflectional $U(z)$ velocity defect at the streak flank (wake-like instability, e.g. Swearingen \& Blackwelder 1987).

First, note that Orr-Sommerfeld inflection-point theory (i.e. necessary conditions for instability), valid strictly for one-dimensional velocity profiles, does not directly apply to the linear stability equations (5) and (6) for a two-dimensional $U(y, z)$ base flow. Secondly, the dominant sinuous mode instability studied here can occur without any $U(y)$ inflections of the streak base flow (1), for which Kelvin-Helmholtz-type instability of localized $U(y)$ shear is thus not the cause. Finally, although strong localized streak-crest $U(y)$ shear is necessary for varicose instability, these modes are stable for streaks typical of near-wall turbulence (see SH).

We now address the possible analogy between (sinuous) streak and wake instabilities, qualitatively suggested by the resemblance of the inflectional $U(z)$ velocity defect through the streak to a one-dimensional wake flow (see also the typical streak $\partial u / \partial z$ profile in figure 6). For this, instability modes are calculated for the one-dimensional sinusoidal wake

$$
U_{\text {wake }}(z)=U_{0}+(\Delta u / 2) \cos \left(\beta_{s} z\right),
$$

matched to the $z$-profile of the streak base flow (1) at $y^{+}=25$ (i.e. $U_{\text {wake }}(z)=U\left(y^{+}=\right.$ $25, z)$ ). The wake modes are calculated using the DNS-based stability analysis outlined in $\S 2.3$, via a spectral simulation code developed for free mixing layers (see Schoppa et al. 1995 for algorithm details and validation). To permit direct comparison, sinuous wake modes are calculated for a wake flow $U_{\text {wake }}(z)$ corresponding to streaks with $2 \pi / \beta_{s}^{+}=100$ and amplitude $\left.\Omega_{y}^{+}\right|_{\max }=0.35$ (figure $2 c$ ), for the streamwise wavelength of $2 \pi / \alpha^{+}=315$.

In figure 8 , the normal vorticity perturbation $\omega_{y}^{\prime}$ of the sinuous streak insta- 

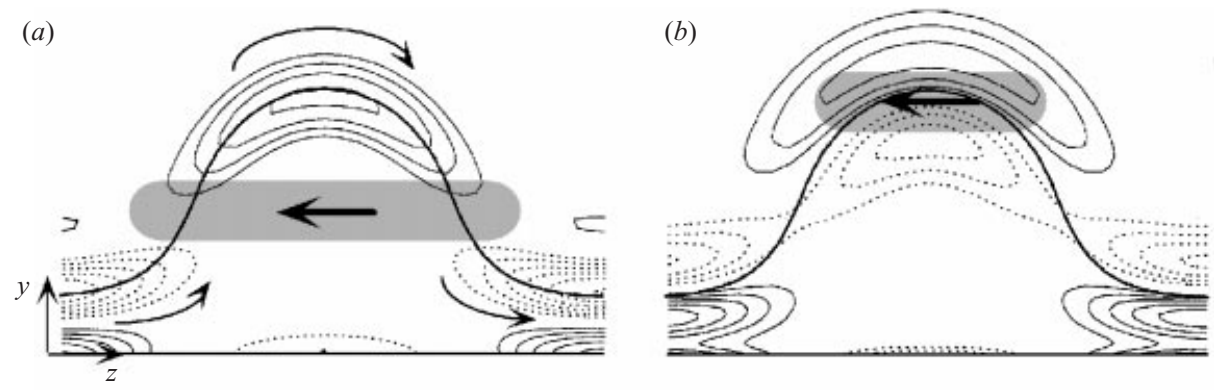

(c)
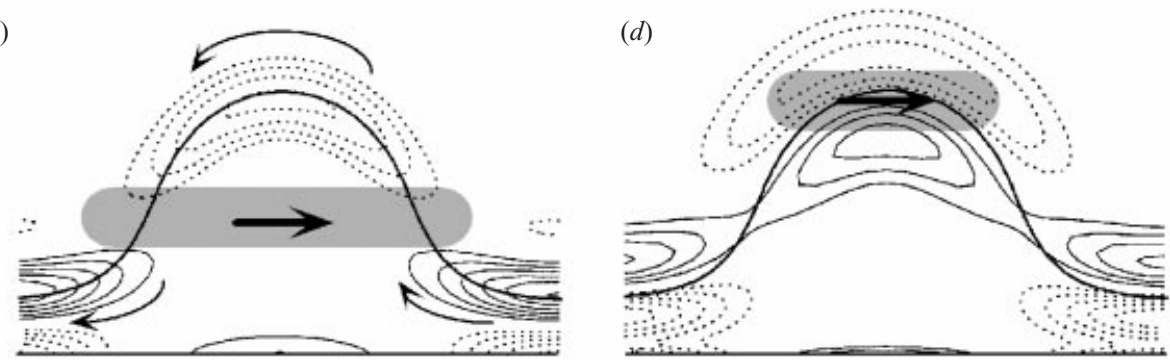

FIGURE 9. Perturbation streamwise vorticity $\omega_{x}^{\prime}$ for sinuous streak instability mode, at $(a) \alpha x=0$, (b) $\alpha x=\pi / 2,(c) \alpha x=\pi$, and $(d) \alpha x=3 \pi / 2$. Positive and negative $\omega_{x}^{\prime}$ are shown as solid and dotted contours respectively, and the bell-shaped line denotes the phase speed contour $U=\sigma_{i} / \alpha$. The shading shows the regions of induced spanwise flow (in the direction of the thick arrow).

bility mode (at $y^{+}=25$; figure $8 a$ ) is compared to that of the corresponding one-dimensional wake mode (figure $8 b$ ). Interestingly, the sinuous wake and streak instability modes have a fundamentally different vorticity structure, despite a very similar base flow $U(z)$. In particular, note that the streak mode is characterized by thin sheets of $\omega_{y}^{\prime}$, which induce nearly-parallel $u^{\prime}$ but weak spanwise motion $w^{\prime}$ due to the sheet-like structure (e.g. parallel, straight $\omega_{y}^{\prime}$ sheets induce no $\left.w^{\prime}\right)$. In contrast, the wake mode consists of elliptical cells of $\omega_{y}^{\prime}$ (figure $8 b$ ), responsible for all induced $w^{\prime}$ due to the mode's two-dimensionality. Additionally, the streak mode $\omega_{y}^{\prime}$ is maximal at the streak flanks (i.e. at $z$-location of maximum $\partial U / \partial z$ ), whereas the maxima of wake $\omega_{y}^{\prime}$ occur within the low- and high-speed regions (i.e. at the $z$-location of zero mean shear). Such fundamental differences in the correspondings $\omega_{y}^{\prime}$ distributions indicate that the streak and wake sinuous $w^{\prime}$ disturbances are generated by distinctly different mechanisms. That is, despite requiring sufficient magnitudes of streak lift angle $\theta_{20}$ and hence $U(z)$ shear, the sinuous streak instability mechanism is not in reality 'wake-like'.

In contrast to the purely two-dimensional wake instability (i.e. containing $\omega_{y}^{\prime}$ only), the streak instability mechanism we find is inherently three-dimensional (dynamics described in §4.4). In particular, the sinuous streak instability mode is characterized by curved sheets of $\omega_{x}^{\prime}$ in the cross-stream $(y, z)$-plane (figure 9), localized primarily near the low-speed streak 'crest' and the high-speed 'trough' regions. Due to their $z$-elongation, these $\omega_{x}^{\prime}$ sheets mainly induce quasi-parallel $w^{\prime}$ within the low-speed streak (shaded regions in figure 9). For example, $-w^{\prime}$ is induced within the streak below the $+\omega_{x}^{\prime}$ layer in figure $9(a)$, and $-w^{\prime}$ is generated at the streak crest between the opposite-signed, dipole-like layers in figure $9(b)$; and vice versa for $+w^{\prime}$ induction in figures $9(c, d)$ due to $x$-antisymmetry. Therefore, sinuous $w^{\prime}(x)$ motion occurring 


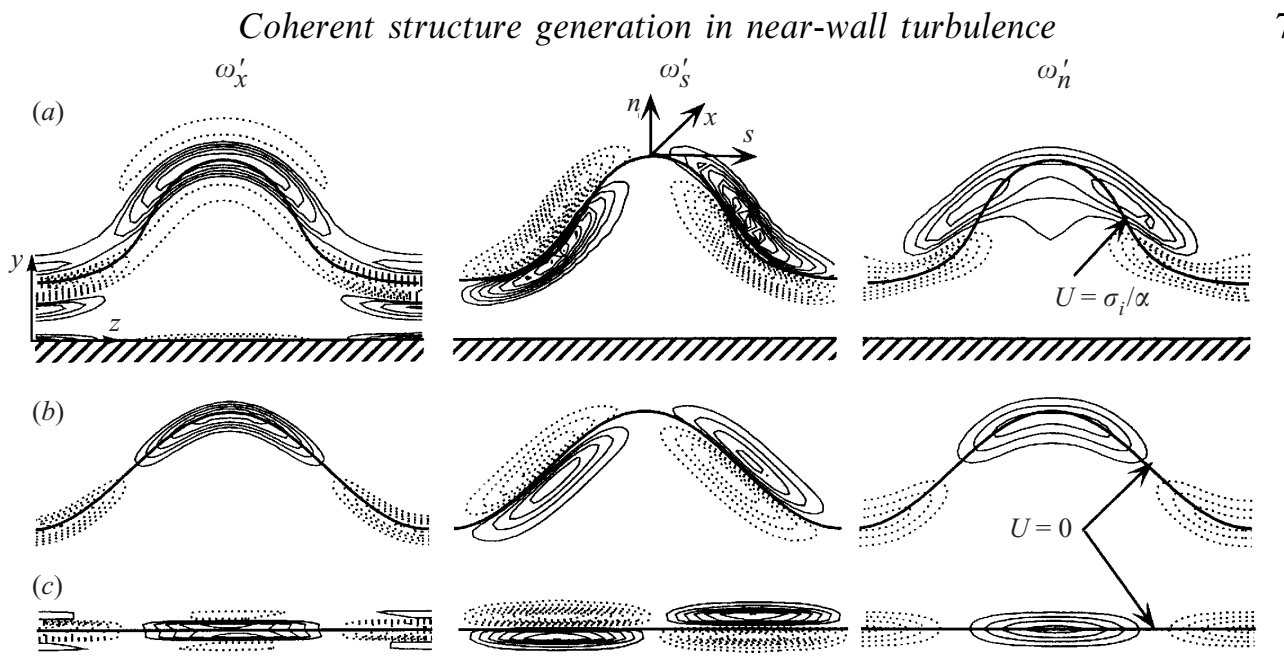

FIGURE 10. Perturbation vorticity distributions in the base-flow vortex-line coordinate system $(x, n, s)$, for sinuous instabilities of $(a)$ near-wall streaks and $(b)$ a corrugated mixing layer, compared with (c) superposed $\pm \beta$ oblique modes of a planar mixing layer. The three columns show $(y, z)$-plane contours of $\omega_{x}^{\prime}, \omega_{s}^{\prime}, \omega_{n}^{\prime}$.

for streak instability is induced predominantly by $\omega_{x}^{\prime}$ sheets, instead of by $\omega_{y}^{\prime}$ as in the (two-dimensional) wake case.

The $\omega_{x}^{\prime}$ sheets are slightly inclined to the wall (in the $(x, y)$-plane) and oppositesigned regions overlap in $x$, reminiscent of the overlapping of counter-rotating longitudinal coherent structures educed from fully developed near-wall turbulence (Jeong et al. 1997). Note, however, that the linear-mode $\omega_{x}^{\prime}$ distribution does not reflect vortices, as the sheet-like geometry induces a predominantly planar wall-parallel flow (i.e. mainly $w^{\prime}(y)$ shear). Furthermore, the even $z$-symmetry of $\omega_{x}^{\prime}$ (figure 9) across the streak is not representative of observed streamwise vortices, which tend to appear on only one streak flank, both in instantaneous realizations and as ensemble-averaged coherent structures. Thus, linear growth of sinuous streak instability is itself not sufficient to explain the generation of observed near-wall vortices. As shown herein, nonlinear effects acting on these streak disturbances (as they evolve into the nonlinear regime) are actually responsible for the observed geometry of near-wall vortices.

Having demonstrated that streak instability is distinct from either localized KelvinHelmholtz or wake instabilities, we now illustrate a close relationship existing with the three-dimensional instability of a planar mixing layer. To understand this connection, it is useful to project the perturbation vorticity onto a local coordinate system referenced to base flow vortex lines (bell-shaped, equivalent to $U=$ constant contours for $x$-independent flows). In particular, defining local binormal $(x)$, tangential $(s)$, and normal $(n)$ coordinates as in figure $10(a)$, the corresponding vorticity components may be transformed as

$$
\left.\begin{array}{l}
\omega_{b}=\omega_{x}, \\
\omega_{s}=-\omega_{y} \frac{\Omega_{y}}{|\Omega|}-\omega_{z} \frac{\Omega_{z}}{|\Omega|}, \\
\omega_{n}=-\omega_{y} \frac{\Omega_{y}}{|\Omega|}+\omega_{z} \frac{\Omega_{y}}{|\Omega|},
\end{array}\right\}
$$

where $\left(\Omega_{y}, \Omega_{z}\right)$ are the $(y, z)$ components of the base flow vorticity with (non-zero) local magnitude $|\Omega|$; note $\Omega_{x}=0$. 
For the special case of a one-dimensional $U(y)$ mixing layer, the $\left(\omega_{b}, \omega_{n}, \omega_{s}\right)$ vorticity components reduce to the usual $\left(\omega_{x}, \omega_{y}, \omega_{z}\right)$ components. In figure $10(c)$, perturbation vorticity contours are shown for superposed $\pm \beta$ oblique modes of a planar tanh mixing layer (see Schoppa et al. 1995 for further details), with streamwise and spanwise wavenumbers chosen to match the most unstable sinuous streak mode. Application of the transformation (11) to the streak mode (figure 10a) reveals a striking correspondence with the mixing-layer oblique modes, for all perturbation vorticity components. That is, by 'lifting' the oblique mode distributions in figure 10(c) at their $z$-midpoint, perturbation vorticity distributions very similar to those of the streak mode (figure $10 a$ ) are obtained. Note also the similar vorticity distributions with respect to the phase speed contour $U=\sigma_{i} / \alpha$ (on which both distributions are constant in time; $\sigma_{i}=0$ for the oblique modes). For instance, $U=\sigma_{i} / \alpha$ passes through the maxima of $\omega_{x}^{\prime}$ and $\omega_{n}^{\prime}$, and separates positive and negative $\omega_{s}^{\prime}$ in both cases.

To progress further, we also investigate a new type of shear layer instability-a $z$-periodic 'corrugated' mixing layer of the form

$$
U_{\text {corr }}(y, z)=\Delta U \tanh (y+A \cos (\beta z))
$$

with a $y$-displacement of amplitude $A$ and a spanwise wavenumber $\beta$ designed to closely match the height and spanwise spacing of lifted streak vortex lines. As shown in figure $10(b)$, sinuous instability modes of the corrugated mixing layer (12) even more closely match the streak instability perturbation vorticity, capturing spatial variations due to the shear-layer curvature as well. Note that some details differ, e.g. opposite-signed $\omega_{n}^{\prime}$ contours overlap (in $n$ ) for the streak mode (figure $10 a$ ), but not for the shear-layer modes (figures $10 b, c$ ). These and other minor vorticity differences result from the $y$-antisymmetry of the corrugated shear layer, not exhibited in the streak base flow (e.g. unlike mixing layers, see lack of $y$-symmetry in figure $2 c$ ).

The close correspondence of the perturbation vorticity distributions in figure $10(a-c)$ indicates a strong connection between streak instability and three-dimensional instability modes of both corrugated and planar mixing layers. A further consequence is that the streak instability is not only inviscid in nature, but the no-slip and impermeability conditions at the wall evidently play no important destabilizing role. That is, viscous effects do not directly enter into the mechanism through the wall conditions. Viscous effects, however, enter into the problem via self-annihilation of the (base flow) streak-flank vorticity by viscous cross-diffusion (discussed in $\S 3.5$ below). In summary, sinuous (normal-mode) streak instability and oblique mixing layer modes share the following features:

(i) Individual oblique modes of a planar mixing layer exhibit a spanwise-wave-type perturbation (e.g. figure $3 c$ ) which is prohibited for $z$-symmetric streaks. However, superposed $\pm \beta$ oblique modes of equal strength (i.e. a standing wave) produce a close correspondence with the streak mode.

(ii) The spanwise phase of perturbations is fixed relative to the $z$-symmetry locations for both streak and corrugated mixing layer instabilities, while this phase is arbitrary for the oblique modes of a planar mixing layer.

(iii) Streak instability is seen to involve the same underlying instability mechanism (discussed in §4.4) as mixing layer oblique modes, noting the close correspondence of all perturbation vorticity components in figure 10 .

\subsection{Streak diffusion effect: unfrozen base flow}

Up to this point, we have focused on normal-mode sinuous streak instability, with the base flow streaks (1) frozen to isolate the instability growth mechanism from the 

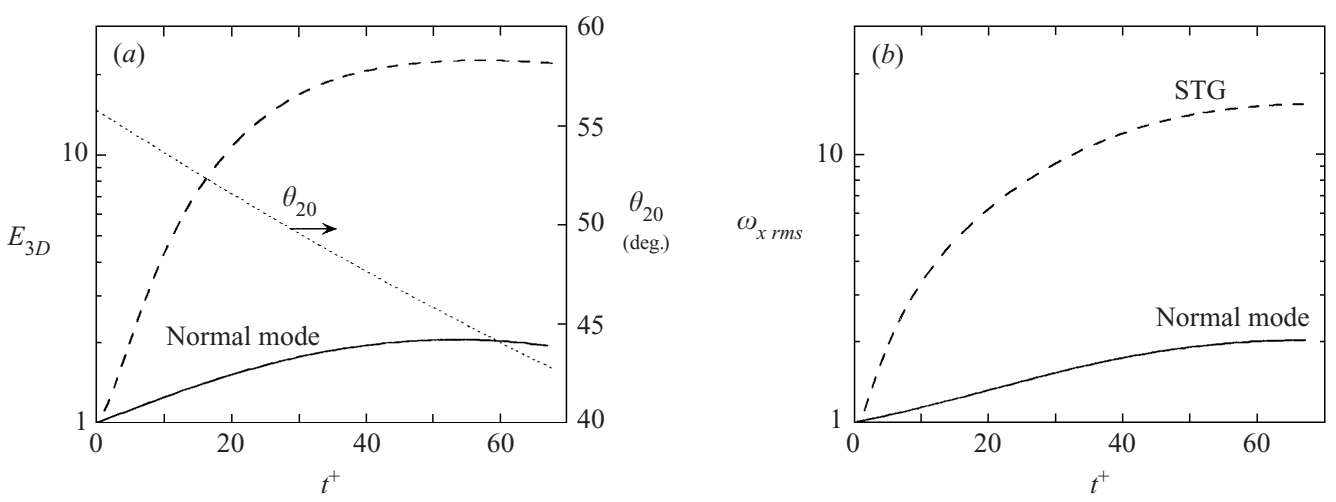

FIGURE 11. Linear evolution of $(a) E_{3 D}$ energy (normalized by initial disturbance energy) and $(b)$ $\omega_{x r m s}$ at $y^{+}=20$ (normalized by the initial value), for the most unstable normal mode (solid) and STG (dashed). All curves are for freely diffusing (unfrozen) streaks, with an initial streak lift angle of $\theta_{20}=56^{\circ}$. Also shown in $(a)$ is the decay of $\theta_{20}$ (dotted; right ordinate) due to streak self annihilation.

effects of base flow vorticity diffusion (i.e. viscous decay of streak $\Omega_{y}(y, z)$ and $\Omega_{z}(y, z)$ ). In situations where the base flow evolves (e.g. via vorticity diffusion) on a much slower timescale than the instability growth, the base-flow evolution is inconsequential (as for planar mixing-layer stability analysis). However, for streaks representative of near-wall turbulence, the base-flow diffusion timescale and instability growth rate are found to be comparable (see below), necessitating analysis of perturbation evolution with a freely evolving ('unfrozen') base flow. Having identified linear instability of frozen streaks, we now consider the linear evolution of an unstable normal mode in the presence of unfrozen, viscously decaying streaks.

Noting the inviscid instability mechanism (see $\mathrm{SH}$ ), in which viscous effects and the no-slip wall play no destabilizing role, the question remains: How do viscous effects-obviously important for the near-wall scaling but non-essential to the growth mechanism-enter the streak instability dynamics? We find that the viscous diffusion of the streak base flow $U(y, z)$ is significant, for the well-accepted streak spacing of $L_{z}^{+}=100$. In particular, the opposite-signed wall-normal (base-flow) vorticity $\Omega_{y}$ flanking the low-speed streak undergoes viscous self-annihilation via cross-diffusion, a kind of planar reconnection. Consequently, $\Omega_{y}$ decays exponentially as $\Omega_{y}=\Omega_{y}(0) \mathrm{e}^{-\gamma t}$, with a non-dimensional (wall-unit) decay rate of $\gamma^{+}=\gamma v / u_{*}^{2}=0.0068$ calculated via DNS. The vorticity (viscous) decay rate for streaks of infinite $y$ extent (i.e. without the wall) can be derived from the vorticity equation for $\Omega_{y}$ applied to the spanwise spacing, and is found to be $\left.v \beta_{s}^{2}\right)^{+}=0.0037$, indicating that roughly half of the $\Omega_{y}$ diffusion flux occurs in $z$ via cross-diffusion. The remaining flux occurs in $y$ primarily to the wall due to the $\Omega_{y}=0$ condition at the wall (considering the diffusion of scalar $\Omega_{y}$ ). Note that the (viscous) streak decay rate $\gamma^{+}=0.0068$, though slower than the streak's (normal-mode) instability growth rate $\left(\sigma_{r}^{+}=0.012\right.$ at $\left.\theta_{20}=56^{\circ}\right)$, is of comparable magnitude, indicating that the streak diffusion is non-negligible.

As a consequence of streak $\Omega_{y}$ diffusion (automatically accounted for in DNS), the streak lift angle $\theta_{20}$ decays as $\theta_{20}(t)=\tan ^{-1}\left[\Omega_{y}(0) \mathrm{e}^{-\gamma t} /\left(\mathrm{d} U_{0} / \mathrm{d} y\right)\right]$, the decay constituting a significant stabilizing effect on streak instability. For instance, an initially unstable streak with $\theta_{20}=56^{\circ}$ reaches the stability cutoff of $\theta_{20}=50^{\circ}$ by $t^{+}=$ 30 , due to streak diffusion (figure 11a). Consequently, growth of the most unstable (normal) sinuous eigenmode (for $\theta_{20}=56^{\circ}$ ), initialized along with an unfrozen streak, 
is rather quickly shut off by the viscous streak decay (see figure 11a). In particular, growth of the instability mode is limited to a factor-of-two amplification of $E_{3 D}$ (volume-integrated energy of all $x$-dependent Fourier modes), after which the streak becomes too weak for linear instability to continue. Note that this 'saturation' and susbsequent decay of $E_{3 D}$ due to streak self-annihilation occurs at a linear amplitude (i.e. instability mode disturbance amplitude near machine accuracy) and thus should not be confused with the (typical) nonlinear saturation.

\section{Linear streak transient growth}

In contrast to the limited growth of the most unstable normal mode of streak instability - a factor-of-two $E_{3 D}$ amplification-we develop here an alternative scenario: the streak transient growth (STG) mechanism. Although both normal-mode instability and STG eventually reach linear 'saturation' (by the countering effect of viscous selfannihilation of streak vorticity), STG has a much higher short-time growth rate and is capable of producing much higher amplification than the most unstable normal mode. Significantly, STG also produces order-of-magnitude perturbation growth for stable streaks, which are far more numerous (i.e. about $80 \%$ of near-wall streaks are stable; see figure $7 b$ ). For the disturbance level prevailing in developed near-wall turbulence, STG causes a sufficiently large perturbation amplitude to excite a nonlinear $\omega_{x}$ amplification mechanism (described in $\S 5.2$ ), resulting in generation of streamwise vortices. Thus, both the normal-mode and STG mechanisms are linear in their growth and decay, STG becoming more effective after large linear amplification, through eventual nonlinearity. We will provide insight into the (fairly elusive) 'physical mechanism' of STG through vortex-dynamics-based reasoning, but we first describe the key features and significance of STG.

Although not specifically applied to date to vortex generation in near-wall turbulence, the concept of (linear) transient growth (see Trefethen et al. 1993) has been used extensively to address the related problem of (subcritical) 'bypass' transition of the mean velocity $U(y)$ (Butler \& Farrell 1992; Reddy \& Henningson 1993). The essential point is that transient growth of disturbances is possible for non-self-adjoint operators like the linearized Navier-Stokes operator for shear flows. Recall that, because of this feature of the linear operator governing perturbation evolution, eigenmodes of the traditional normal mode analysis are not orthogonal to one another. Because of this non-orthogonality, particular disturbances (say, specific combinations of normal modes) can generally be amplified by significant factors (by transient growth), even if all constituent normal modes are individually stable! For the most energetic perturbation of $U(y)$, transient growth generates an $x$-independent streak via the well-known 'lift-up' mechanism. Note, however, that for such an $x$-independent flow, streamwise vorticity decays monotonically by self-annihilation, and thus no sustained turbulence can occur. In contrast, for the new transient growth scenario developed here, we focus on growth of $x$-dependent disturbances, necessary for $\omega_{x}$ generation, and hence for subsequent generation of near-wall streamwise vortices and 'bursts' of Reynolds stress and turbulence production. The near-wall streamwise vortices - the footprints of which were initially termed 'bursts' (Kline et al. 1967) - are considerably shorter in $x$ (by an order of magnitude) than low-speed streaks (figure 1; see also Jeong et al. 1997 ) and can result only from three-dimensional, $x$-dependent perturbations. Additionally, note that we consider perturbations of $U(y, z)$ streaks, not to be confused with prior transient growth studies of the one-dimensional, streakless mean velocity $U(y)$. 
We first present evidence of transient growth of streak perturbations $(\S 4.1)$, illustrating the dominance of STG over normal-mode instability. An explanation of the underlying STG mechanism is outlined via vortex dynamics arguments in $\S 4.2$, followed by additional explanation in the context of energy considerations ( $\$ 4.3)$. The distinction of the normal-mode streak instability mechanism from STG is described in $\S 4.4$.

\subsection{STG: observations}

Since most near-wall streaky regions are stable and the amplification of (infrequently occurring) normal-mode-unstable streaks is highly limited, a different mechanism must be responsible for the successive (re)generation of near-wall streamwise vortices. We thus consider transient growth of streak perturbations as an alternative mechanism which: (i) causes significant perturbation growth (much higher than normal-mode instability) for freely diffusing streaks, and (ii) operates for weaker, normal-modestable streaks, found to be much more common near the wall (figure $7 b$ ).

With the goal of elucidating the significance of transient growth for streak $U(y, z)$ base flows, we consider a spanwise velocity perturbation of the form

$$
w^{\prime}(x, y)=W \sin (\alpha x) g(y),
$$

with $g(y)=y \exp \left(-\eta y^{2}\right)$ and a linear amplitude $W$; hereinafter, (13) is referred to as the $S T G$ perturbation. This simple initial perturbation is motivated by a number of physical considerations. First, as will be shown in $\S 4.2$, growth of streamwise vorticity $\omega_{x}^{\prime}$ is driven by the combined effect of streak shear (predominantly $\partial U / \partial y$ ) and $x$-variation of $w^{\prime}$ through a new 'shearing' mechanism of vorticity generation. Second, $x$-dependence of $w^{\prime}$ is also required for production of $u w$ Reynolds stress for extraction of kinetic energy by the perturbation, as shown in $\S 4.3$. Analysis in $\S 4.3$ shows that a simple spanwise flow perturbation (i.e. as given by (13)) normal to the streak-flank $\Omega_{y}$ generates the (dominant) velocity perturbation $u^{\prime}$ (i.e. $u_{x}^{\prime}$ ), and hence can extract kinetic energy from the ( $x$-averaged) mean flow more rapidly than possible in normal-mode instability. Finally, analysis of fully developed near-wall turbulence indeed confirms that sinuous streak displacement motion like (13) is prevalent and precedes the generation of streamwise vortices (discussed in $\S 5.1$ ).

The non-normality of the STG perturbation may be easily verified: given (13), there is no value of $\sigma$ for which the perturbation equations (6) can be satisfied. Unlike traditional normal-mode instability (i.e. linear growth leading to nonlinear saturation), the peak perturbation amplitude resulting from transient growth depends directly on its initial amplitude. That is, while linear growth of normal modes typically saturates (due to nonlinear effects) at virtually the same amplitude independent of the initial amplitude - a characteristic feature of normal modes - the final amplitude reached by transient growth does depend strongly on the initial amplitude. Hence, it is important to analyse perturbations which are prevalent in developed near-wall turbulence, as captured by (13).

Frozen streak. Note that transient growth is characterized by rapid temporal perturbation growth (for a base flow which can be unstable or even stable to normal modes). To clearly demonstrate the significance of STG, we first consider the case of a frozen $U(y, z)$ streak (i.e. with no base-flow evolution), in order to isolate the growth phenomenon from the suppressive effect of viscous streak self-annihilation in unfrozen (freely evolving) flows. Additionally, we focus on STG via analysis of a linearly stable streak, say with $\theta_{20}=45^{\circ}$, as an illustrative example (i.e. a streak which produces no growth of normal modes; see figure $4 b$ ). Significantly, we find that the 

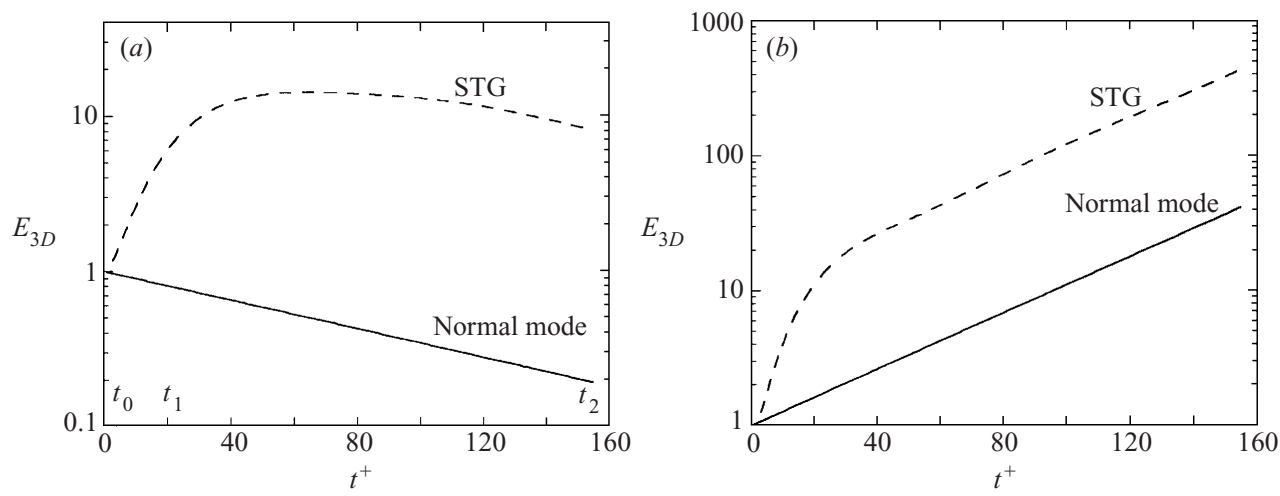

FIGURE 12. Comparison of normal mode and transient growth of linear three-dimensional perturbation energy, for a frozen base flow (i.e. streak diffusion not permitted): (a) normal-mode-stable streaks with $\theta_{20}=45^{\circ} ;(b)$ normal-mode-unstable streaks with $\theta_{20}=56^{\circ}$.

STG perturbation (13) does in fact produce significant (factor of 10) amplification of $E_{3 D}$ (figure $12 a$ ), in contrast to the exponential decay of the least-stable normal mode. Subsequently, the STG perturbation linearly 'saturates' and eventually decays, as discussed in $\S 4.3$. The significance of this large, albeit temporary, (linear) amplification of energy in $x$-dependent modes lies in the consequence that nonlinear effects are triggered at high amplitudes - the nonlinearity following the large amplification due to linear STG-leading to streamwise vortex generation ( $(5.2)$. The transient growth mechanism thus reveals a dynamically active role of stable streaks, found to be more prominent in near-wall turbulence than unstable streaks.

For the (less frequently occurring) unstable streaks, STG also plays a dominant role, producing more rapid early-time growth and larger amplification than the most unstable normal mode. As an illustration, consider an unstable streak (e.g. with $\left.\theta_{20}=56^{\circ}\right)$, for which STG produces an early-time $E_{3 D}(t)$ growth which is significantly faster than the most unstable normal mode $\left(t^{+}<20\right.$ in figure $\left.12 b\right)$, resulting in a factor of 10 larger amplification. This faster early-time growth rate, compared to normal-mode instability is due to (subtle) differences between the perturbation vorticities of STG and normal modes, described in $\S 4.2-\S 4.4$ below.

Unfrozen streak. Returning to the physically relevant case of an unfrozen (freely diffusing) streak, STG generates a factor-of-20 amplification of $E_{3 D}$, even in the presence of countering streak diffusion (figure 11a). In contrast, the corresponding (initially unstable) normal mode experiences only a factor-of-two $E_{3 D}$ growth, for the same streak distribution $U(y, z)$. Growth of both STG and the normal mode is eventually shut off (past $t^{+} \sim 50$ ) by the streak being progressively weakened by the ever-present vorticity self-annihilation (reflected by $\theta_{20}$ in figure $11 a$ ). Until the time of this linear 'saturation', STG generates an order-of-magnitude larger amplification than the initially unstable normal mode, due to the faster early-time growth mechanism of STG (described below). STG also produces much more significant growth of streamwise vorticity, critical for near-wall vortex generation. For instance, a factorof-16 amplification of $\omega_{x r m s}$ occurs for STG (at $y^{+}=20$ ), compared to only a factor of two for the most unstable normal mode (figure $11 b$ ).

As an aside, similar disturbance growth for unstable streaks in SH (the distinction from STG was not addressed there) is shown to be self-similar in $R e$ over the range 2650-10650. The self-similarity was shown by the collapse of the growth rate as 

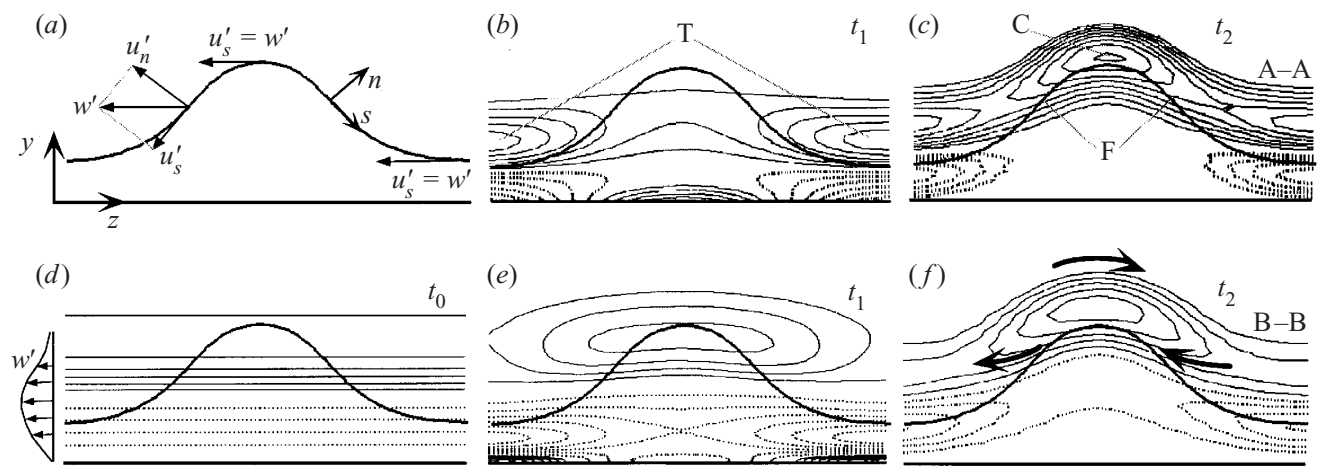

$(g)$

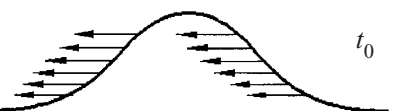

(h)

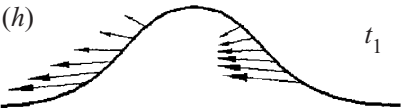

(i)

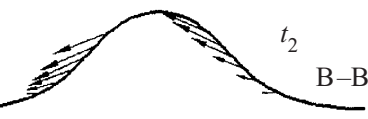

FIGURE 13. Evolution of perturbation $(b-f) \omega_{x}$ and $(g-i)$ streak-flank velocity vectors during STG; these distributions are in planes $\mathrm{A}-\mathrm{A}$ and $\mathrm{B}-\mathrm{B}$ identified in figure $14:(b, c)$ in plane $\mathrm{A}-\mathrm{A}$ and $(d-i)$ in plane B-B. The times correspond to $(d, g) t_{0},(b, e, h) t_{1}$, and $(c, f, i) t_{2}$. The streak vortex line coordinate system is defined in $(a)$. Regions $\mathrm{C}, \mathrm{T}$, and $\mathrm{F}$ denote the crest, trough, and flank of the streak, respectively.

a function of time, both non-dimensionalized in wall units. That is, for self-similar scaling of the base-flow streaks, the wall-normal vorticity $\Omega_{y}$ increases with increasing $R e$, as does the rate of streak self-annihilation. A concomitant increase in disturbance growth rate occurs with increasing $R e$ due to the correspondingly higher streak-flank vorticity (e.g. see increasing growth rate with $\theta_{20}$ in figure $4 b$ ). We find that the two effects-instability growth and streak self-annhilation-continue to be in balance as $R e$ is increased. Although perhaps not totally surprising from the (highly debated) viewpoint that outer-scale events play an unimportant role in the near-wall dynamics, this $R e$-scaling suggests that the same growth behaviour occurs at practical Reynolds numbers, with autonomous near-wall (i.e. inner-scaling) dynamics. This prompted $\mathrm{SH}$ to assert the prominence of the same perturbation growth mechanism and associated DNS results at much higher Re values, beyond the reach of DNS.

\subsection{STG: streamwise vorticity generation mechanism}

Up to this point, we have demonstrated the dominance of STG in generating orderof-magnitude growth of $E_{3 D}$ and $\omega_{x}$, for both stable and unstable streaks. We now address the underlying physical-space (perturbation) vortex dynamics, focusing on STG of the more frequently occurring normal-mode-stable streaks, for which the growth mechanism can be clearly revealed and distinguished from normal-mode instability. Superficially, the premise of perturbation growth for otherwise normalmode-stable base flows may appear enigmatic. The principal question addressed in this section is: how does transient growth of streak perturbations occur physically, when all normal modes decay (e.g. figure 12a)?

Perturbation vorticity equation. To explain the underlying mechanism of STG, we focus on the dynamics of $\omega_{x}^{\prime}$ generation, via vorticity perturbation equations derived for the streak-vortex-line coordinate system $(x, n, s)$ defined in figure 13(a). Recall that the $s$ and $n$ directions are everywhere tangent and normal, respectively, to the base-flow vortex lines (equivalently, constant- $U$ contours for $x$-independent flow). A key advantage of this base-flow vortex-line coordinate system is that it significantly 


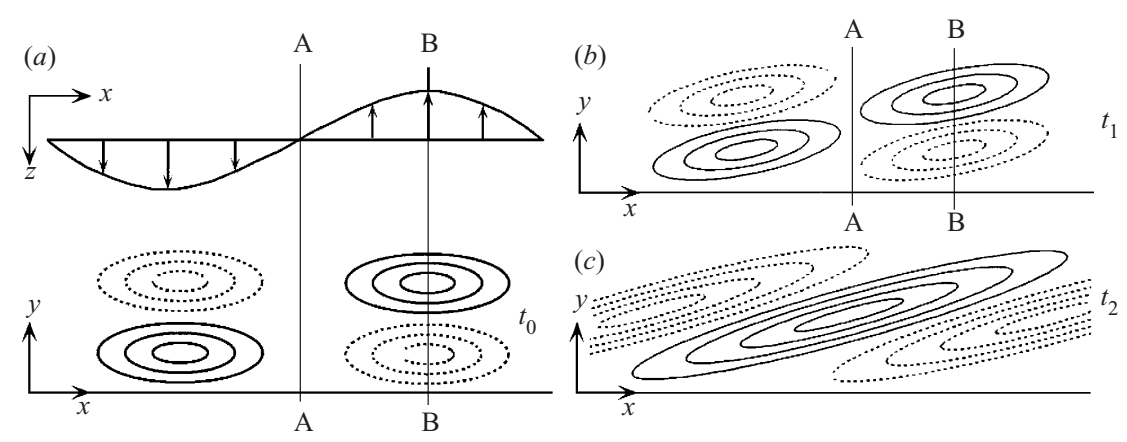

FIGURE 14. Evolution of perturbation $\omega_{x}^{\prime}$ during STG, in the $(x, y)$-plane passing through the streak creast, at times $(a) t_{0},(b) t_{1}$, and $(c) t_{2}$. Solid and dotted contours denote $+\omega_{x}^{\prime}$ and $-\omega_{x}^{\prime}$, respectively. The $(y, z)$ planes A-A and B-B are defined in $(a)$.

simplifies the evolution equations for perturbation vorticity and enables simpler physical interpretations. In particular, both the base-flow velocity $U$ and vorticity $\Omega$ are single-component (i.e. $\boldsymbol{u}=U(n) \boldsymbol{x} ; \boldsymbol{\omega}=\Omega(n, s) s$ ), and $U$ is one-dimensional in this coordinate system. Transforming the vorticity equation into $(x, n, s)$ coordinates (Lamb 1945) for linearized perturbations of a $U(n)$ streak distribution, the inviscid evolution equations for vorticity perturbations $\left(\omega_{x}^{\prime}, \omega_{n}^{\prime}, \omega_{s}^{\prime}\right)$ are derived as

$$
\begin{gathered}
\frac{\partial \omega_{x}^{\prime}}{\partial t}+U \frac{\partial \omega_{x}^{\prime}}{\partial x}=h_{s} \Omega \frac{\partial u_{x}^{\prime}}{\partial s}+h_{n} \omega_{n}^{\prime} \frac{\mathrm{d} U}{\mathrm{~d} n}=\Omega \frac{\partial u_{s}^{\prime}}{\partial x}, \\
\frac{\partial \omega_{n}^{\prime}}{\partial t}+U \frac{\partial \omega_{n}^{\prime}}{\partial x}+\underbrace{h_{s} h_{n} \Omega u_{n}^{\prime} \frac{\partial\left(1 / h_{n}\right)}{\partial s}}=h_{s} \Omega \frac{\partial u_{n}^{\prime}}{\partial s}, \\
\frac{\partial \omega_{s}^{\prime}}{\partial t}+U \frac{\partial \omega_{s}^{\prime}}{\partial x}+\underbrace{h_{s} u_{s}^{\prime} \frac{\partial \Omega}{\partial s}}+h_{n} u_{n}^{\prime} \frac{\partial \Omega}{\partial n}=h_{s} \Omega \frac{\partial u_{s}^{\prime}}{\partial s}+\underbrace{h_{s} h_{n} \Omega u_{n}^{\prime} \frac{\partial\left(1 / h_{s}\right)}{\partial n}},
\end{gathered}
$$

where the direction cosine amplitudes $h_{n}$ and $h_{s}$ are determined from

$$
h_{n}^{2}=\left(\frac{\partial n}{\partial y}\right)^{2}+\left(\frac{\partial n}{\partial z}\right)^{2}, \quad h_{s}^{2}=\left(\frac{\partial s}{\partial y}\right)^{2}+\left(\frac{\partial s}{\partial z}\right)^{2} .
$$

The base-flow vorticity is given by $\Omega=-h_{n} \mathrm{~d} U / \mathrm{d} n$ in transformed coordinates, which permits simplification of the two $\omega_{x}^{\prime}$ production terms (first two terms on the righthand side of (14a)) to a single term. This simplification of the right-hand side of the $\omega_{x}^{\prime}$ equation is important in understanding the underlying transient growth mechanism, in that physical-space interpretation of the original tilting terms is obscured by their partial cancellation of each other (i.e. rewriting the second tilting term as $h_{n} \omega_{n}^{\prime} \mathrm{d} U / \mathrm{d} n=\Omega \partial u_{s}^{\prime} / \partial x-\Omega h_{s} \partial u_{x}^{\prime} / \partial s$, the first tilting term $\Omega h_{s} \partial u_{x}^{\prime} / \partial s$ is cancelled). Alternatively, interpretation of the sole $\omega_{x}^{\prime}$ generation term $\Omega \partial u_{s}^{\prime} / \partial x$ clearly reveals the essential mechanism of STG-differential advection ('shearing') of perturbation velocity by the mean shear-which differs from the traditional concepts of vorticity tilting and stretching, as explained below.

To understand the dynamics of $\omega_{x}^{\prime}$ generation by STG, we focus throughout this section on normal-mode-stable streaks with $\theta_{20}=40^{\circ}$, as an illustrative example. (The reader is forewarned that figures 13-16 are interrelated and must be discussed together for clarity.) The perturbation evolution characteristic of STG is illustrated in figures 13 and 14 by $(y, z)$ - and $(x, y)$-distributions of $\omega_{x}^{\prime}$, respectively; the $(y, z)$ 

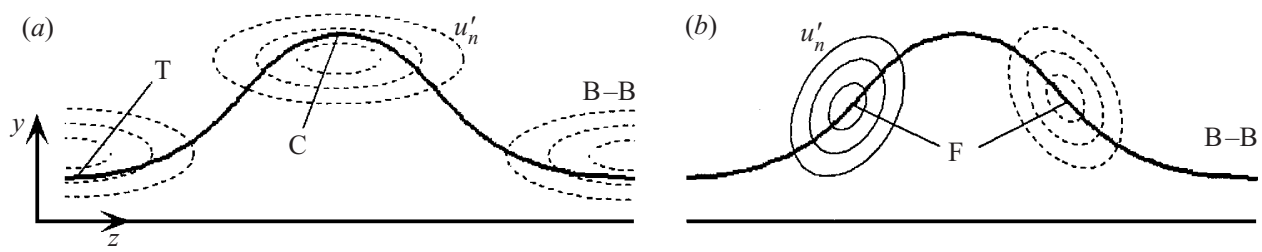

FiguRE 15. Schematic of $(a) u_{s}^{\prime}$ and $(b) u_{n}^{\prime}$ distributions near the streak creast $(\mathrm{C})$, trough $(\mathrm{T})$, and flanks (F) for the initial STG perturbation in plane B-B, obtained via projection of (13) onto streak vortex-line coordinates $(n, s)$. Positive and negetive contours are solid and dashed, respectively.

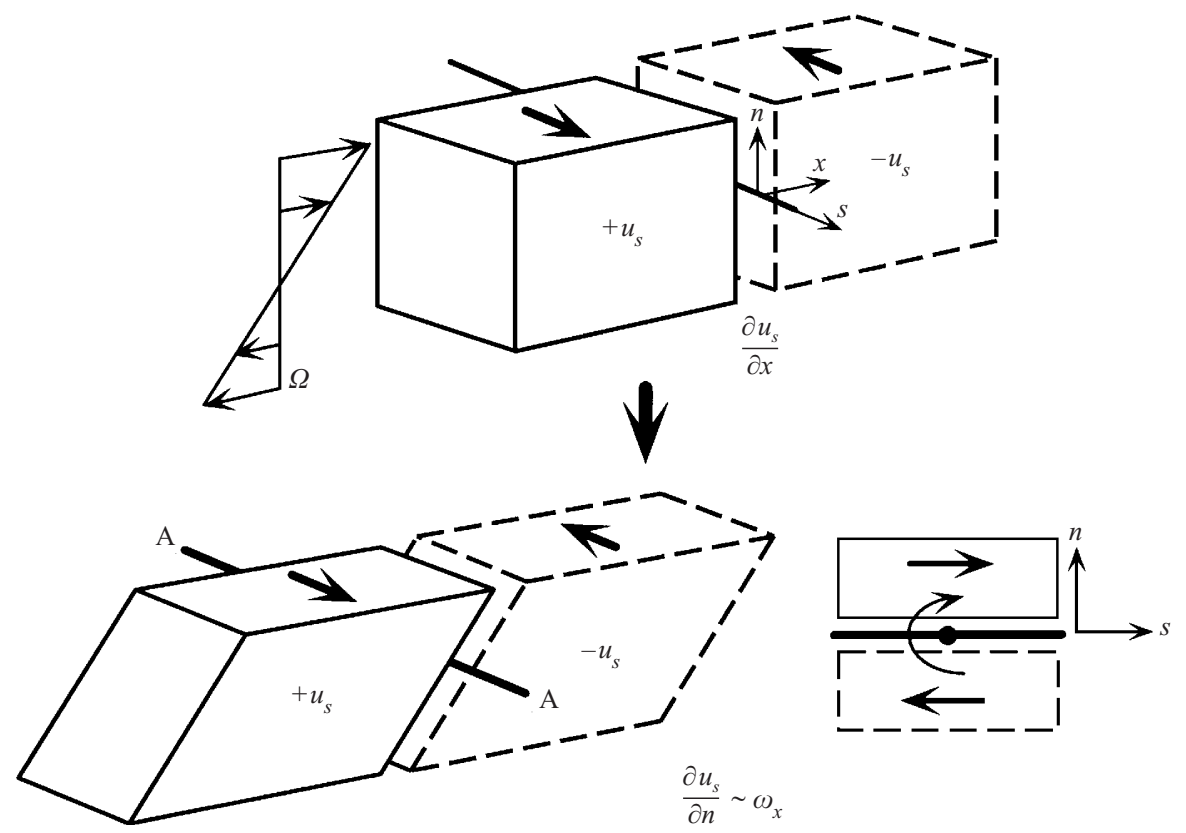

FIGURE 16. Physical mechanism of 'shearing' generation of vorticity, i.e. $\omega_{x}^{\prime}$ production due to the term $\Omega \partial u_{s}^{\prime} / \partial x$. This new mechanism is responsible for vorticity generation in both STG and normal-mode streak instability (as well as corrugated mixing-layer instability).

cross-sections $\mathrm{A}-\mathrm{A}$ and $\mathrm{B}-\mathrm{B}$ are defined based on the $x$-symmetry of STG. The initial STG perturbation velocity, decomposed in vortex line coordinates, is shown in figure 15. The shearing mechanism of $\omega_{x}^{\prime}$ generation by the base flow is illustrated schematically in figure 16 .

Initial $S T G$ perturbation. Initially at $t_{0}$ (i.e. $t=0$ ), the $\omega_{x}^{\prime}$ contours are wallparallel straight lines in B-B, reflecting the wall-jet-like spanwise flow $w^{\prime}(y)$ of the STG perturbation (figure 13d). Due to the sinusoidal $x$-variation of the perturbation (13), $\omega_{x}^{\prime}$ is characterized by overlapping cells with $x$-alternating sign, with maximum amplitude in B-B but identically vanishing $\omega_{x}^{\prime}$ in A-A (figure 14a). In terms of perturbation velocity, STG initially consists of the $u_{s}^{\prime}$ and $u_{n}^{\prime}$ distributions shown in figures $15(a, b)$, with maximum amplitude in $\mathrm{B}-\mathrm{B}$ and a sinusoidal $x$-variation. The components $u_{s}^{\prime}$ and $u_{n}^{\prime}$ are obtained via projection of the STG $w^{\prime}$-perturbation onto the $n$ - and $s$-directions, as illustrated in figure $13(a)$. Note that the $u_{s}^{\prime}$ componentshown below to be critical for $\omega_{x}^{\prime}$ generation-is maximum in the streak crest $(\mathrm{C})$ and trough $(\mathrm{T})$ regions, where the STG perturbation $w^{\prime}$ is aligned with streak vortex 
lines (i.e. with the $s$-coordinate; figure $15 a$ ). In contrast, the $u_{n}^{\prime}$ component (velocity perturbation normal to streak vortex lines) is localized to the streak flank (F) regions (figure $15 b$ ). As will be shown in $\S 4.3, u_{n}^{\prime}$ is critical for production of perturbation kinetic energy.

Early-time $S T G$ evolution. Immediately after $t_{0}, \omega_{x}^{\prime}$ is rapidly generated in A-A, producing by $t_{1}$ (i.e. $t^{+}=20$ ) a flat, $z$-localized elliptical patch of $+\omega_{x}^{\prime}$ in the streak trough region ( $\mathrm{T}$ in figure $13 \mathrm{~b}$ ). The essential mechanism of STG, responsible for this early-time $\omega_{x}^{\prime}$ growth, is reflected by the sole $\omega_{x}^{\prime}$ generation term $\Omega \partial u_{s}^{\prime} / \partial x$ in (14). To see this, first note that $u_{s}^{\prime}$ is largest in magnitude in plane $\mathrm{B}-\mathrm{B}$; due to the sinusoidal $x$-variation of STG, $\partial u_{s}^{\prime} / \partial x$ is thus largest in magnitude in plane A-A. Secondly, recall that $u_{s}^{\prime}$ and hence $\partial u_{s}^{\prime} / \partial x$ are maximum in the streak trough and crest regions (figure $15 a$ ). Further, the $\Omega \partial u_{s}^{\prime} / \partial x$ generation term is selectively larger in the streak trough, where the streak shear $|\Omega=| \mathrm{d} U / \mathrm{d} n \mid$ is maximum (being much weaker in the crest). Collectively, the action of the (base-flow) streak shear $\Omega$ on the perturbation $\partial u_{s}^{\prime} / \partial x$ leads to significant $\omega_{x}^{\prime}$ generation (via the term $\Omega \partial u_{s}^{\prime} / \partial x$ in (14)), in the form of a flat, $z$-localized patch of $+\omega_{x}^{\prime}$ in the streak trough region in plane A-A (figure 13b). Note that the $-\omega_{x}^{\prime}$ regions in figure 13(b) (underneath $+\omega_{x}^{\prime}$, closer to the wall) are a kinematic consequence of the wall no-slip condition, due to the presence of $u_{s}^{\prime}$ generated near the wall.

'Shearing' generation of vorticity. The physical mechanism by which $\partial u_{s}^{\prime} / \partial x$ generates $\omega_{x}^{\prime}$ within a local shear (i.e. with mean vorticity $\Omega$ ) is illustrated in figure 16 . The regions of $u_{\mathrm{s}}^{\prime}$ of the STG perturbation (13) are represented in the top panel, which alternate in sign in $x$ accordingly. Due to the base flow shear $\mathrm{d} U / \mathrm{d} n$, the initially rectangular $u_{s}^{\prime}$ regions are shear-deformed into a parallelogram-shape, with $+u_{s}^{\prime}$ advected over the top of $-u_{s}^{\prime}$ (at line $\mathrm{A}-\mathrm{A}$ in figure 16). Consequently, a spanwise shear layer $u_{s}^{\prime}(n)$ with streamwise vorticity $+\omega_{x}^{\prime}$ is generated (bottom-right panel) in the region where $\partial u_{s}^{\prime} / \partial x$ is negative. By symmetry, $-\omega_{x}^{\prime}$ is generated at a half- $x$ wavelength away, where $\partial u_{s}^{\prime} / \partial_{x}$ is positive. Note that the streak-vortex-line coordinate system permits this simplified interpretation of perturbation vorticity generation, distinct both in functional form and physical interpretation from the typical concept of vorticity tilting (as well as its more rigorous analytical representation; Panton 2001).

Late-time STG evolution. Subsequent to early-time STG growth, $+\omega_{x}^{\prime}$ is similarly generated in $\mathrm{A}-\mathrm{A}$ along the entire streak periphery, albeit more slowly in regions of lesser shear (i.e. the streak crest $\mathrm{C}$ and flanks $\mathrm{F}$ ), eventually resulting in a $z$-continuous, lifted $+\omega_{x}^{\prime}$ sheet by $t_{2}$ (figure $13 c$ ). Note the unexpected result that the peak $\omega_{x}^{\prime}$ at the crest is even higher than at the trough (region of highest shear), presumably because of more rapid cross-diffusion of overlapping, opposite-signed $\omega_{x}^{\prime}$ in the trough region (due to closer proximity to the wall). In $\mathrm{B}-\mathrm{B}$, the $\omega_{x}^{\prime}$ evolution is dominated by simple $x$-advection via the $U \partial \omega_{x}^{\prime} / \partial x$ term in $(14 a)$. In particular, $+\omega_{x}^{\prime}$ generated near $\mathrm{A}-\mathrm{A}$ is advected overhead of $-\omega_{x}^{\prime}$ generated a half $x$-wavelength away, resulting in $z$-elongated sheets of 'lifted' $\omega_{x}^{\prime}$ (figure $13 f$ ), with the $x$-elongated, opposite-signed $\omega_{x}^{\prime}$ sheets overlapping in $y$ in $\mathrm{B}-\mathrm{B}$ (figure $14 c$ ).

In this interpretation of $\omega_{x}^{\prime}$ generation, note that growth rate of $\omega_{x}^{\prime}$ directly depends on the magnitude of $\partial u_{s}^{\prime} / \partial x$. For STG of normal-mode-stable streaks, $u_{s}^{\prime}(x)$ is initially imposed, which causes early-time $\omega_{x}^{\prime}$ generation before viscous decay. That is, the creation of $x$-overlapping $u_{s}^{\prime}$ layers of opposite signs by the base flow shear (figure 16) eventually results in accelerated viscous decay of $u_{s}^{\prime}$ and hence of $\partial u_{s}^{\prime} / \partial x$. Thus, after the initial transient growth, $\omega_{x}^{\prime}$ generation via the continually decaying $\partial u_{s}^{\prime} / \partial x$ is eventually unable to overcome the viscous cross-annihilation of opposite-signed, overlapping $\omega_{x}^{\prime}$ sheets (e.g. figure $14 c$ ), resulting in $\omega_{x}^{\prime}$ decay at late times. The 
corresponding energy-based analysis of STG linear saturation appears in $\S 4.3$. Note the distinction with normal modes for unstable streaks, where a coupling of $\omega_{x}^{\prime}$ and $\omega_{n}^{\prime}$ leads to sustained growth of $\partial u_{s}^{\prime} / \partial x$ and hence of $\omega_{x}^{\prime}$, resulting in instability (explained in $\S 4.4)$.

Normal mode lock-on. The late-time $\omega_{x}^{\prime}$ distribution also corresponds to the leaststable normal mode, due to the inherent lock-on of arbitrary disturbances to the corresponding normal mode. That is, for an arbitrary initial disturbance (including perturbations susceptible to transient growth) consisting of a linear combination of normal modes, the least-stable normal mode will either grow fastest or decay slowest, and hence will emerge as the dominant perturbation as $t \rightarrow \infty$. STG of linearly stable streaks is necessarily temporary (as implied by 'transient'), as the STG perturbation must eventually evolve into the least-stable normal mode (with the same underlying dynamics of $\left.\omega_{x}^{\prime}\right)$ at late times. Note that in contrast to normal modes, whose spatial distribution is fixed (with only time-varying amplitude), the perturbation vorticity distribution evolves continuously during STG. Hence, the fundamental distinction between STG and (stable) normal modes is illustrated by the qualitatively different early-time $\left(t_{1}\right)$ and late-time $\left(t_{2}\right) \omega_{x}^{\prime}$ distributions in figures 13 and 14. Consider figures $13(b)$ and 13(c) representing STG and the stable normal mode: STG produces more $z$-localized and flattened $\omega_{x}^{\prime}$ patches in the streak trough, while the normal mode produces a $z$-continuous, lifted $\omega_{x}^{\prime}$ sheet. In the $(x, y)$ plane, STG generates $x$-compact $\omega_{x}^{\prime}$ cells (figure 14b), compared to $x$-elongated $\omega_{x}^{\prime}$ layers for the normal mode (figure 14c).

\subsection{STG: energy production mechanism}

Having described the underlying dynamics of $\omega_{x}^{\prime}$ generation during STG, we now consider the corresponding mechanism of energy growth. We begin with the customary evolution equation for the perturbation kinetic energy $E$, simplified for $(x, z)$-periodic disturbances $\bar{u}^{\prime}$ of the base flow $U(y, z)$ and a base flow vortex-line coordinate system (defined above):

$$
\frac{\mathrm{d} E}{\mathrm{~d} t}=\underbrace{\int_{V}-\left(u_{x}^{\prime} u_{n}^{\prime} h_{n} \frac{\mathrm{d} U}{\mathrm{~d} n}\right) \mathrm{d} V}_{P}-\underbrace{\frac{1}{R e} \int_{V}\left(\nabla \boldsymbol{u}^{\prime} \cdot \nabla \boldsymbol{u}^{\prime}\right) \mathrm{d} V}_{\varepsilon} ; \quad E \equiv \int_{V} \frac{1}{2}\left(\boldsymbol{u}^{\prime} \cdot \boldsymbol{u}^{\prime}\right) \mathrm{d} V .
$$

In the following discussion, the energy production $P$ and dissipation $\varepsilon$ are normalized by the kinetic energy $E$. The energy growth rate $(1 / E) \mathrm{d} E / \mathrm{d} t\left(=2 \sigma_{r}\right.$ for a normal mode) is the difference between $P$ (generally positive) and $\varepsilon$ (strictly positive). Unlike (linear) normal modes, for which $P$ and $\varepsilon$ are constant in time, STG is characterized by temporal variation of both $P$ and $\varepsilon$. Since the physical-space distribution of nonnormal perturbations evolves temporally (as explained above), the 'efficiency' $P / \varepsilon$ of a given perturbation in generating energy growth (i.e. $P / \varepsilon>1$ ) generally varies in time during transient growth.

As an illustrative example, we consider STG for normal-mode-stable streaks with $\theta_{20}=40^{\circ}$, for which the evolutions of energy production $P$ and dissipation $\varepsilon$ are shown in figure 17. Note that there is no production $P$ at $t_{0}$, since the initial STG perturbation (13) contains no $u_{x}^{\prime}$, resulting in $P=0$ from (16). Immediately thereafter, $P$ sharply increases and significantly surpasses the perturbation's $\varepsilon$, resulting in rapid transient growth of $E$ for $t^{+}<30$ (figure 17). Subsequently, a rapid decrease in $P$ coupled with a gradual increase in $\varepsilon$ results in energy saturation at $t^{+} \sim 50$ (when $P / \varepsilon \sim 1)$ and decay thereafter. Note that both $P$ and $\varepsilon$ approach time-asymptotic 


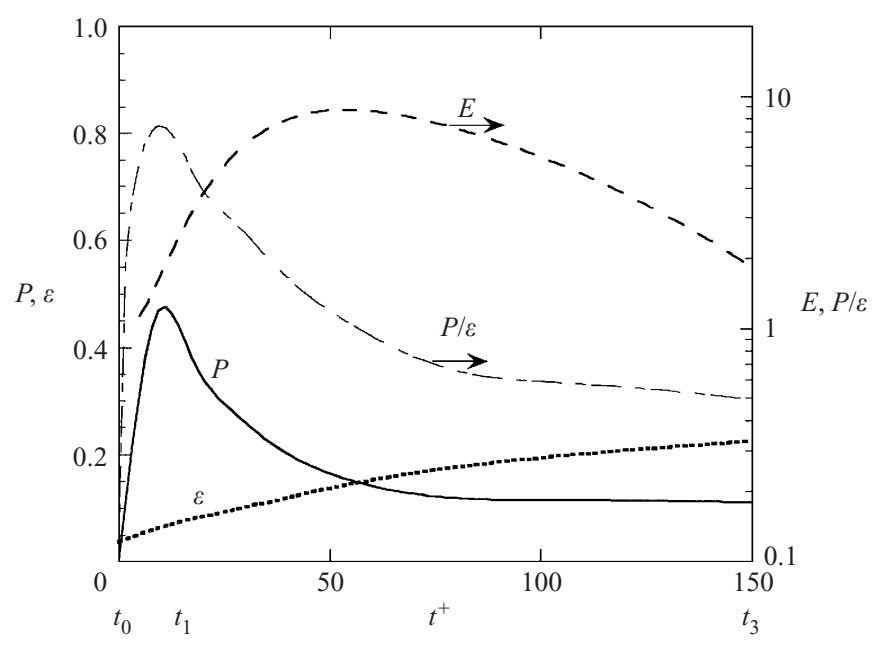

FIGURE 17. Temporal evolution of perturbation kinetic energy $E$, production $P$, dissipation $\varepsilon$, and the 'perturbation efficiency' $P / \varepsilon$ during STG, for normal-mode-stable streaks with $\theta_{20}=40^{\circ}$. Note that both $P$ and $\varepsilon$ are normalized by the instantaneous value of $E$.
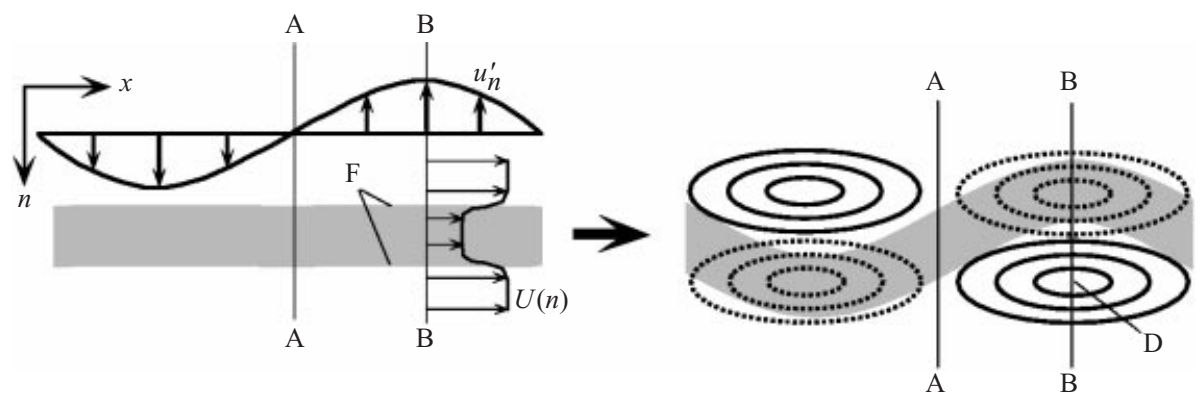

FIGURE 18. Schematic of STG energy growth mechanism, illustrating streak $z$-shifting mechanism of $u_{x}^{\prime}$ generation, with positive (solid) and negative (dashed) $u_{x}^{\prime}$ values shown in relation to the sinuously deforming streak.

(constant) values (figure 17), reflecting the late-time 'lock-on' of the perturbation to the corresponding least-stable normal mode, with $P / \varepsilon<1$ indicating its stability.

Energy production mechanism. The sharp increase in energy production $P$ characteristic of STG (figure 17) is driven solely by the Reynolds stress $-u_{x}^{\prime} u_{n}^{\prime}$ in (16), which transfers energy from the streak base flow $U(n)$ to the perturbation. As a visual interpretation of this production mechanism, figure 18 shows the STG $u_{n}^{\prime}(x)$ perturbation of the streak, with the maximum $\mathrm{d} U / \mathrm{d} n$ occurring on either streak flank $F$. Due to the advection of the base flow vorticity $\mathrm{d} U / \mathrm{d} n$ by the $u_{n}^{\prime}(x)$ perturbation, rapid growth of $u_{x}^{\prime}$ occurs on the streak flanks, given by $\partial u_{x}^{\prime} / \partial t \sim-h_{n} u_{n}^{\prime} \mathrm{d} U / \mathrm{d} n$. To understand this $u_{x}^{\prime}$ growth, consider a point D in plane B-B where the STG-induced $z$-shifting of the streak is maximum. The streak movement in $z$ brings in high-speed fluid to $\mathrm{D}$, thus increasing $u_{x}^{\prime}$ there. Hence, the production $P$ in (16) is maximum within the $u_{x}^{\prime}$ cells (e.g. within region D). This constitutes the essential physical-space energy growth mechanism of STG. Consequently, during early-time evolution (i.e. between $t_{0}$ and $t_{1}$ ) in which strong streak-flank $u_{n}^{\prime}$ persists (figure $13 g, h$ ), large $P$ and hence rapid growth of $E$ occur.

STG linear saturation. Having demonstrated a rather simple mechanism of energy 
production during STG, the question arises: why does growth eventually cease? For this, we return to the curved $\omega_{x}^{\prime}$ sheets generated at late times by the 'shearing' mechanism of vorticity generation, described in $\S 4.2$. Focusing on figure 13 at late times $\left(t_{2}\right)$, the induced velocity of the curved $\omega_{x}^{\prime}$ sheet (figure $13 f$ ) at the streak crest is predominantly along the streak-flank vortex line (i.e. dominated by $u_{s}^{\prime}$; figure $13 i$ ). This late-time generation of curved $\omega_{x}^{\prime}$ sheets thus decreases $u_{n}^{\prime}$, the component normal to the vortex lines. Hence follows a decline of $P$ below $\varepsilon$, resulting in the saturation and decay of $E$. Note that perturbation dissipation $\varepsilon$ is dominated by the $\left(\partial u_{x}^{\prime} / \partial z\right)^{2}$ contribution, arising from the opposite-signed $u_{x}^{\prime}$ cells across the streak (figure 18). Owing to this saturation mechanism inherent to STG, the (linear) saturation level depends on the initial perturbation amplitude, which has implications for the onset of nonlinearity (see $\S 5.1$ ).

\subsection{Normal mode instability mechanism}

Corrugated mixing layer. In order to clearly distinguish the unique characteristics of STG, we now describe the analogous growth mechanism for unstable normal modes. Owing to the close correspondence of sinuous streak and corrugated shear-layer instability modes (figure 10), we analyse here the more tractable former case to reveal the underlying streak instability mechanism. The corrugated mixing-layer base flow (12) is particularly useful in that it permits analytical representation of the base-flow vortex-line coordinate transformation $(x, y, z) \rightarrow(x, n, s)$, defined (in closed form) as

$$
\left.\begin{array}{l}
n=y+A \cos (\beta z), \\
s=(2 / \beta) \tan ^{-1}\left[\tan (\beta z / 2) \mathrm{e}^{A \beta^{2} y}\right], \quad 0 \leqslant \beta z \leqslant \pi, \\
s=(2 / \beta) \tan ^{-1}\left[\tan (\beta z / 2) \mathrm{e}^{A \beta^{2} y}\right]+2 \pi / \beta, \quad \pi<\beta z \leqslant 2 \pi,
\end{array}\right\}
$$

where the functional form of the $s$ definition is chosen to ensure its boundedness and continuity across $\beta z=n \pi$. Furthermore, to permit direct comparison with oblique modes of a planar mixing layer, the coordinate transformation is defined so that $(n, s)$ reduces to $(y, z)$ as the corrugation amplitude $A \rightarrow 0$. It is easily verified that, as $A \rightarrow 0$, the governing equations (14) reduce to those for oblique modes of a planar mixing layer, and the terms underlined with braces in (14) vanish identically. In the light of the strong resemblance of the corrugated and planar mixing-layer modes (figure 10), these underlined terms, representing the effects of the base-flow curvature, are thus non-essential to the instability mechanism (thus not considered further). To further simplify the analysis, we focus on the perturbation vorticity evolution in the centreplane $n=0$; note that simple $x$-advection of vorticity (e.g. the $U \partial \omega_{x}^{\prime} / \partial x$ term in $(14 a))$ vanishes identically in this plane, on which $U=0$.

$\omega_{n}^{\prime}$ generation. The coordinate transformation and simplifications developed here reveal an easily tractable physical mechanism of instability, driven predominantly by a coupling between vorticity perturbations $\omega_{x}^{\prime}$ and $\omega_{n}^{\prime}$, each augmenting the other (hence instability). Focusing first on $\omega_{n}^{\prime}$ generation, the $(n, s)$ distribution of the sole generation (tilting) term $h_{s} \Omega \partial u_{n}^{\prime} / \partial s$ (right-hand side of (14b); figure 19a) directly corresponds to the $\omega_{n}^{\prime}$ distribution at the same $x$ (figure $10 b$ ), hence indicating that $\omega_{n}^{\prime}$ generation via tilting is dominant (i.e. overshadowing advection terms on the lefthand side of $(14 b)$ ). The physical mechanism of the $\omega_{n}^{\prime}$ generation term $h_{s} \Omega u_{n}^{\prime} / \partial s$ is illustrated in figure $20(a)$ via $\omega_{x}^{\prime}$-contours at $x_{0}$ and $x_{\pi}$ (i.e. a half-wavelength apart in $x$ ), with corresponding $u_{n}^{\prime}$ profiles at $n=0$. Figure $20(b)$ shows the perturbation effect of $\omega_{x}^{\prime}$ on initially spanwise-aligned streak vortex lines; the dashed lines pass through the points of maximum $\left|\omega_{x}^{\prime}\right|$ generation: $\mathrm{P}$ (positive $\omega_{x}^{\prime}$ ) and $\mathrm{N}$ (negative $\omega_{x}^{\prime}$ ). As 

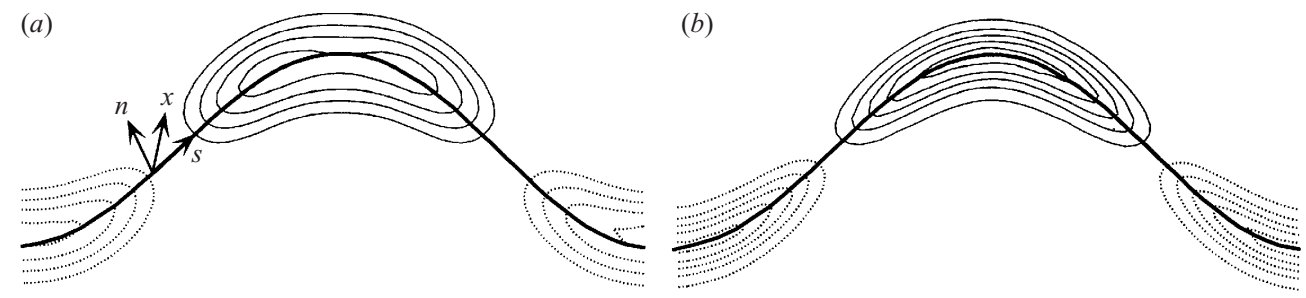

FIGURE 19. Dominent perturbation vorticity production terms for instability of a corrugated mixing layer: (a) $\omega_{n}^{\prime}$ production term $h_{s} \Omega \partial u_{n}^{\prime} / \partial s$ and $(b) \omega_{x}^{\prime}$ production term $\Omega \partial u_{s}^{\prime} / \partial x$. Positive and negative magnitudes are denoted by solid and dotted contours respectively, and the bold line represents the $U=0$ contour.
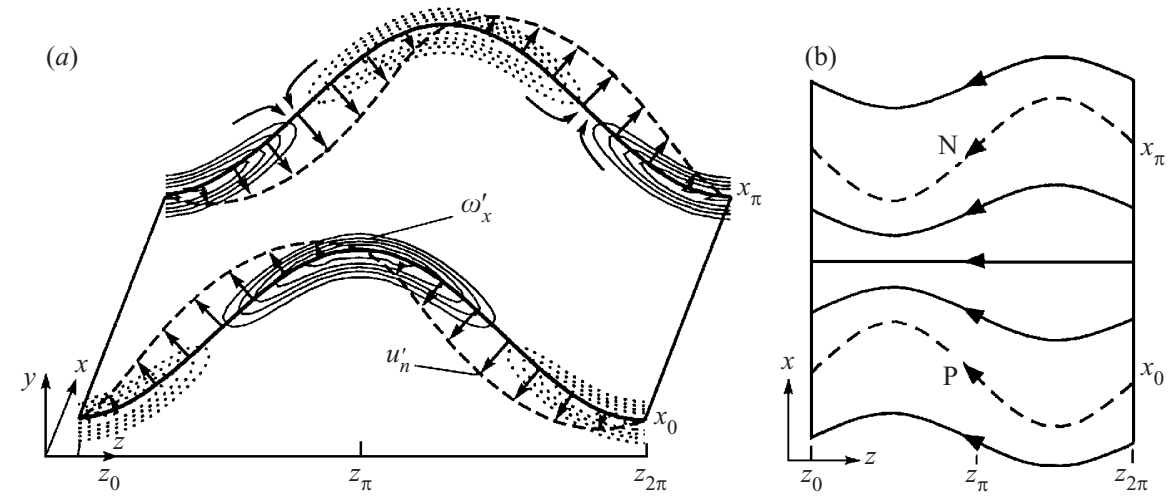

FIGURE 20. Illustration of $\omega_{n}^{\prime}$ production mechanism in $(a)$ perspective view and $(b)$ top view, for corrugated mixing-layer instability. In $(a)$, profiles of $u_{n}^{\prime}$ are overlaid with contours of $\omega_{x}^{\prime}$ at two $x$ values half a wavelength apart. Perturbed vortex lines (initially aligned with $z$ in this view) are shown in $(b)$ and the dashed lines in $(b)$ correspond to those in $(a)$. $\mathrm{P}$ and $\mathrm{N}$ denote points of maxium positive and negetive $\omega_{x}^{\prime}$ generation.

shown, positive and negative $u_{n}^{\prime}$ are induced predominantly by the counter-rotating $\omega_{x}^{\prime}$ regions embedded within the mixing layer with vorticity $\Omega$. That is, positive and negative $u_{n}^{\prime}$ are induced on the left and right sides of the positive $\omega_{x}^{\prime}$ regions (due to their finite $s$-extent); vice versa for negative $\omega_{x}^{\prime}$. Thus, induction of the positive and negative $\omega_{x}^{\prime}$ regions reinforce one another, resulting in jet-like $u_{n}^{\prime}$ ejections out of the mixing layer, with maximum strength between adjacent $\omega_{x}^{\prime}$ layers (i.e. at $z_{\pi / 2}$ and $z_{3 \pi / 2}$ ). Consequently, the base-flow vorticity $\Omega$ (thick vortex lines in figure $20 a$ ) is tilted by the $s$-varying $u_{n}^{\prime}$ to generate $\omega_{n}^{\prime}$ at locations of non-zero $\partial u_{n}^{\prime} / \partial s$. Note that maxima of $\left|\partial u_{n}^{\prime} / \partial s\right|$ and hence $\left|\omega_{n}^{\prime}\right|$ correspond spatially with the $\omega_{x}^{\prime}$ regions (figure 20), due to this dominant role of $\omega_{x}^{\prime}$ induction in the $\omega_{n}^{\prime}$ generation mechanism.

$\omega_{x}^{\prime}$ generation. In turn, we find that $\omega_{x}^{\prime}$ generation is dominated by the $x$-varying perturbation velocity $u_{s}^{\prime}$ induced by the vorticity perturbation $\omega_{n}^{\prime}$. With reference to the evolution equation $(14 a), \omega_{x}^{\prime}$ evolution is dominated by the 'shearing' generation term $\Omega \partial u_{s}^{\prime} / \partial x$ (see physical explanation in $\S 4.3$ ), the dominance inferred by the close correspondence of the spatial distribution (figure 19b) with that of $\omega_{x}^{\prime}$ (figure $10 b$ ). As shown by the normal-mode distributions of $u_{s}^{\prime}$ (vectors) and $\omega_{n}^{\prime}$ (contours) overlaid on the $U=0$ surface in figure 21, $x$-varying $u_{s}^{\prime}$ is a direct consequence of opposite-signed cells of $\omega_{n}^{\prime}$ lying within the shear layer. In particular, positive and negative $u_{s}^{\prime}$ are induced adjacently upstream and downstream of the positive $\omega_{n}^{\prime}$ cells; and vice versa for negative $\omega_{n}^{\prime}$. With reference to figure 21, this $\omega_{x}^{\prime}$ generation effect is maximum 


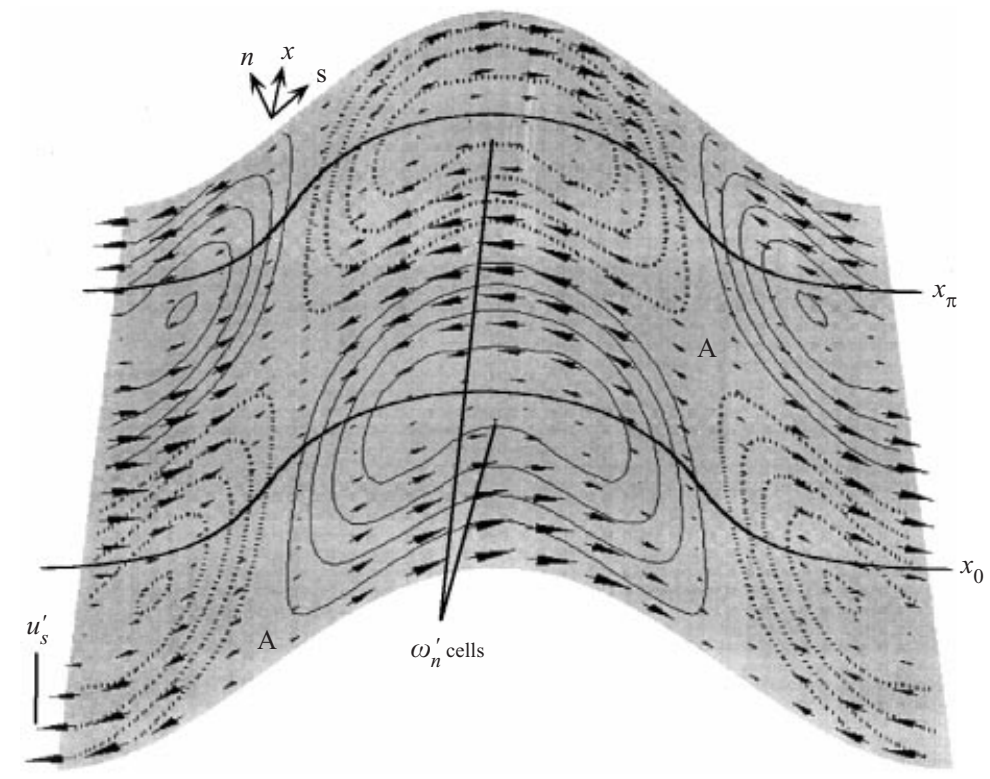

FIGURE 21. Illustration of $x$-varying $u_{\mathrm{s}}^{\prime}$ (vectors) induced by $\omega_{n}^{\prime}$ (contours) for corrugated mixing-layer instability, responsible for $\omega_{x}^{\prime}$ production $\left(U=0\right.$ surface grey-shaded). Vortex lines through the $\omega_{n}^{\prime}$ cells are shown at $x_{0}$ and $x_{\pi}$ and $\mathrm{A}$ denotes regions of maximum $\omega_{s}^{\prime}$ stretching.

within the $\omega_{n}^{\prime}$ cells; note that $\omega_{n}^{\prime}$, in turn, generates the $x$-varying $u_{s}^{\prime}$ required for $\omega_{x}^{\prime}$ production. This closes the coupling between $\omega_{x}^{\prime}$ and $\omega_{n}^{\prime}$.

$\omega_{s}^{\prime}$ generation. In this vortex-dynamics-based physical explanation, tangential vorticity $\omega_{s}^{\prime}$ is seen to play a more indirect role in the sinuous instability mechanism. As illustrated in figure $10(b), \omega_{s}^{\prime}$ appears as sheets of vorticity which alternate in sign across each flank of the corrugated shear layer. Hence, the $\omega_{s}^{\prime}$ distribution simply causes shifting of the streak vortex lines normal to itself at the flanks; e.g. in figure 10(b), both streak flanks are shifted leftward. The relatively large cross-flank peaks of $\omega_{s}^{\prime}$ in figure $10(b)$ are thus a reflection of the large $\Omega$ gradient existing on the streak flank. With reference to figure 20 (at the same location $x_{0}$ ), this shear layer shifting is seen to be a direct consequence of the $u_{n}^{\prime}$ induced by vorticity perturbations $\omega_{x}^{\prime}$ (e.g. compare base flow and perturbed vortex lines). This mechanism of $\omega_{s}^{\prime}$ generation via $n$-displacement (by perturbation velocity $u_{n}^{\prime}$ ) of base-flow vorticity is captured by the vorticity advection term $h_{n} u_{n}^{\prime} \partial \Omega / \partial n$ (left-hand side of $(14 c)$ ). Additional $\omega_{s}^{\prime}$ generation occurs by the stretching term $h_{s} \Omega \partial u_{s}^{\prime} / \partial s$ (right-hand side of $(14 c)$ ), where positive $\partial u_{s}^{\prime} / \partial s$ occurs on the streak flanks (regions A in figure 21). Thus, simultaneous growth of $\omega_{s}^{\prime}$ accompanies instability, but is predominantly a passive consequence of local advection by $\omega_{x}^{\prime}$ - and $\omega_{n}^{\prime}$-induced velocities. This growth of $\omega_{s}^{\prime}$ therefore does not directly feed back onto the coupled $\omega_{x}^{\prime}$ - and $\omega_{n}^{\prime}$-generation mechanism.

In summary, tractable physical mechanisms underlying both normal-mode streak instability and STG are revealed through analysis of perturbation vorticity evolution in a (curvilinear) streak vortex line coordinate system.

Unstable normal modes vs. STG. For normal modes of stronger unstable streaks (i.e. $\theta_{20}>50^{\circ}$ ), an inherent coupling exists between $\omega_{x}^{\prime}$ and $\omega_{n}^{\prime}$ (hence instability), in that (i) $s$-varying $u_{n}^{\prime}$ induced by $\omega_{x}^{\prime}$ generates $\omega_{n}^{\prime}$, and in turn (ii) $x$-varying $u_{s}^{\prime}$ induced by $\omega_{n}^{\prime}$ generates $\omega_{x}^{\prime}$ via 'shearing' vorticity generation. This coupling is consistent with the closely overlapping spatial distributions of $\omega_{x}^{\prime}, \omega_{n}^{\prime}$ (figure $10 b$ ), and the corresponding 
production terms (figure 19). For STG of weaker stable streaks (i.e. $\theta_{20}<50^{\circ}$ ), the normal-mode coupling of $\omega_{x}^{\prime}$ and $\omega_{n}^{\prime}$ is insufficiently strong to overcome viscous self-annihilation. The STG disturbance compensates for the lack of $\omega_{n}^{\prime}$ growth on the streak crest and trough by directly feeding $u_{\mathrm{s}}^{\prime}(x)$ into the shearing mechanism (ii) of $\omega_{x}^{\prime}$ generation. On the streak flanks, the STG disturbance is composed of $u_{n}^{\prime}(x)$, which generates rapid perturbation energy growth via the streak-shifting mechanism reflected in (16). Subsequently, the streak $U(n)$ shear creates overlapping, lifted sheets of $\omega_{x}^{\prime}$, whose curvature strengthens $u_{s}^{\prime}(x)$ on the streak flank at the expense of $u_{n}^{\prime}(x)$, hence halting the STG energy growth mechanism at later times.

Unstable vs. stable normal modes. The $\omega_{x}^{\prime}$ distributions of unstable and stable normal modes (the latter captured by the late-time evolution described in $\$ \$ 4.2$ and 4.3) contain some subtle, yet dynamically significant, qualitative differences. For unstable streaks, the coupling of $\omega_{x}^{\prime}$ and $\omega_{n}^{\prime}$ required for sustained instability growth is localized to the streak crest and trough, resulting in elliptical $\omega_{x}^{\prime}$ regions here (figure $9 a$ ). The opposite sign and $y$-displacement of $\omega_{x}^{\prime}$ regions induce significant $u_{n}^{\prime}$ (primarily $w^{\prime}$; shaded region in figure $9 a$ ) and hence energy production. In contrast, for stable streaks, this $\omega_{x}^{\prime}-\omega_{n}^{\prime}$ coupling is insufficient to sustain perturbation growth, resulting in: (i) $x$-overlapping, lifted $\omega_{x}^{\prime}$ sheets with minimal concentration at the streak crest and trough (figure 13c,f), and (ii) predominant induction of $u_{s}^{\prime}$ (figure 13i), rather than the $u_{n}^{\prime}$ required for production.

\section{Nonlinear streak transient growth and vortex generation}

Up to this point, we have focused on the linear growth of three-dimensional perturbations of lifted streaks, having identified both linear instability of sufficiently strong streaks and a prevalent transient growth mechanism that destabilizes the more numerous normal-mode-stable streaks. We now demonstrate via DNS (including all nonlinear terms) that initially linear perturbation growth due to STG can trigger strong nonlinear effects, even for normal-mode-stable streaks. Significantly, from an initially quiescent streak region containing no initial vortices, nonlinear STG rapidly generates the well-documented structures near the wall: streamwise vortices and internal shear layers, including associated turbulence and Reynolds stress events. (Note that while the 'shearing' generation mechanism is dominant in linear STG, it is not ultimately responsible for the (nonlinear) vortex formation.)

\subsection{Nonlinear growth}

In the following, we isolate STG by initializing linearly stable streaks of the form (1), with a streak lift angle $\theta_{20}=45^{\circ}$ (stability shown in figure $4 b$ ). Hence, any perturbation growth is due to STG, as all normal modes are (linearly) stable in this case. Note that analogous results for linearly unstable streaks (with $\theta_{20}=56^{\circ}$ ) appear in $\mathrm{SH}$, where both the transient growth and normal-mode instability mechanisms contribute to perturbation growth.

Owing to the temporal nature of linear STG-rapid early-time energy growth followed by slower decay - the STG mechanism will not generate significant nonlinear effects if the initial perturbation level is small (dotted curve in figure 22, where the (linear) amplitude is magnified $10^{6}$ times). Recall $(\S 4.3)$ that the inherent shorttime duration of STG is responsible for its linear saturation; thus the saturation level depends on the initial perturbation amplitude. Hence, finite-amplitude initial disturbances are necessary for the tenfold amplification of STG to be large enough to trigger the nonlinear effects by which streamwise vortices are formed. For the 


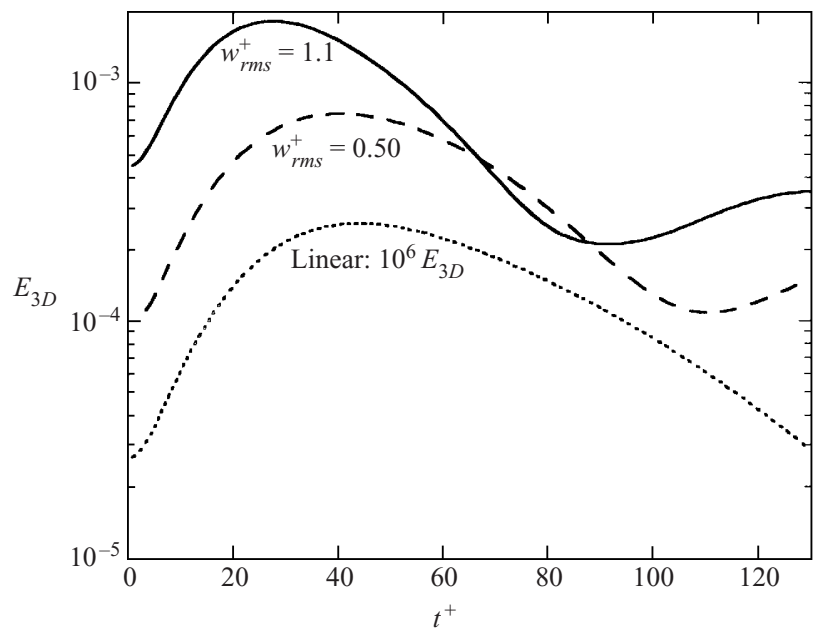

FIGURE 22. Growth of STG perturbation energy into the nonlinear regime, for linearly stable streaks (all with $\theta_{20}=45^{\circ}$ ). The dotted line shows linear STG (magnified $10^{6}$ times). Note that the perturbation amplitude $w_{r m s}^{+}=1.1$ (solid) corresponds to the disturbance level in fully devoloped turbulence.

STG perturbation (13), initialized over the amplitude range $w_{r m s}^{+}=0.5-1.1$, significant $E_{3 D}$ growth occurs well into the nonlinear regime (figure 22). In contrast, similar generation of nonlinearity by normal modes is unlikely, due to the strong limitation of normal-mode growth (only twofold amplification) because of the streaks viscous self-annihilation. The level at which nonlinearity can be said to have set in cannot be defined exactly, but is evident from the nature of disturbance evolution, such as the loss of $z$-symmetry in the perturbation $\omega_{x}$ distribution (exhibited in figure 13b), described in $\S 5.2$ below. In figure 22 , this onset of nonlinearity corresponds to an $E_{3 D}$ value of about 0.0003 , as evident from the large-time trend of $E_{3 D}(t)$. Significantly, nonlinear STG generates indefinitely sustained turbulence (i.e. nonlinear-amplitude $E_{3 D}$ in figure 22), eventually leading to the minimal channel regeneration cycle (with associated $E_{3 D}$ oscillations). Note that sustained turbulence is generated from an even smaller disturbance amplitude if the streaks are stronger (i.e. normal-mode-unstable), e.g. $w_{r m s}^{+}=0.25$ for streaks with $\theta_{20}=56^{\circ}$ (see $\mathrm{SH}$ ).

The STG-based idealized flow analysed here-a (stable) straight streak $U(y, z)$ with a superimposed sinuous $w(x)$ disturbance - is well-representative of $u w$ Reynolds stress behaviour in fully developed turbulence (not to be confused with the extensively studied $u v$ Reynolds stresses). In particular, observations of $u w$ Reynolds stress events of quadrant $Q 2_{u w}\left(u_{t}<0, w_{t}>0\right)$ and quadrant $Q 3_{u w}\left(u_{t}<0, w_{t}<0\right)$ show numerous well-defined $x$-alternating patterns (figure $23 a$; same instantaneous realization as in figure 1). The correspondence of these $Q 2_{u w}$ and $Q 3_{u w}$ events in figure 23(a) with the dynamically significant near-wall CS can be established by comparison with figure 1. The numerous patterns of alternating $Q 2_{u w}$ and $Q 3_{u w}$ events in figure 23(a) agree well with the STG perturbation shown by the insert (figure 23b), both in $(x, z)$ scale and $u w^{+}$amplitude (compare $u w^{+}$contour levels in figures $23 a$ and $23 b$ ). This close agreement indicates that STG-type disturbances-capable of producing order-of-magnitude growth of $E_{3 D}$ and $\omega_{x}$-are prevalent in near-wall turbulence.

The origin of these organized $u w$ patterns can be traced to the induced velocity of: (i) newly created streamwise vortices embedded in the buffer layer, (ii) remnant 


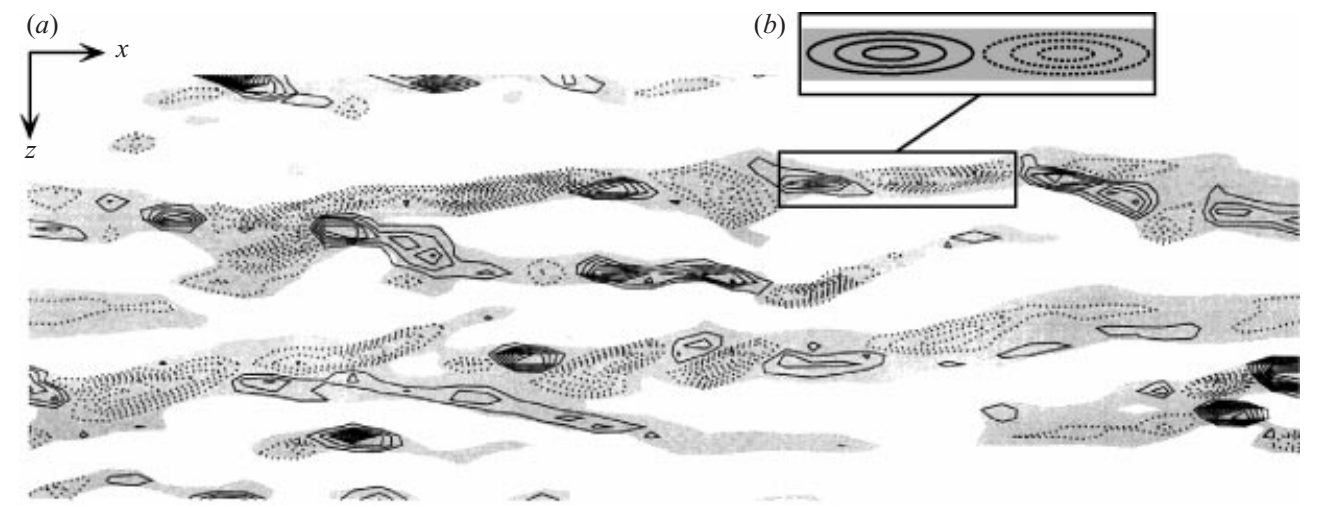

FIGURE 23. $u w$ Reynolds stress events $Q 2_{u w}$ (dotted contours) and $Q 3_{u w}$ (solid contours) in (a) fully developed near-wall turbulence, and $(b)$ idealized flow analysed here, consisting of a straight streak $U(y, z)$ and the STG $w^{\prime}(x)$ disturbance.

streamwise vortices in the buffer region, following near-wall annihilation (via crossdiffusion) of $\omega_{x}$ of neighbouring opposite-signed vortices, and (iii) asymmetric, hookshaped hairpin vortices in the log region. Note that the hairpin legs analyzed in Zhou et al. (1999) commonly exhibit a large inclination from the wall, which in turn can induce a significant $x$-varying $w$ underneath the hairpin leg (in the buffer layer). Each of these induced flows may be a source of the STG $w(x)$ perturbation and subsequent vortex formation studied herein. This scenario serves as an explanation for the creation of new 'quasi-streamwise' vortices underneath and beside pre-existing inclined vortices, observed by Brooke \& Hanratty (1991) and Zhou et al. (1999).

\subsection{Streamwise vortex generation}

\subsubsection{DNS visualization}

The most significant aspect of the STG mechanism outlined here is the formation of new streamwise vortices, once STG grows to nonlinear amplitudes. The streamwise vortex generation process is clearly illustrated by the 'clean' $\omega_{x}$ evolution in figure 24 , free from extraneous perturbations or incoherent turbulence. The initial STG disturbance is characterized by relatively weak layers of $\omega_{x}$ (figure $24 a$ ), the same as for linear STG (figure 13d). After a short period of growth (e.g. $t^{+} \sim 17$, figure $24 b$ ), the $\omega_{x}$ perturbation attains a similar physical-space structure as the linear STG perturbation (figure 13b), indicating the predominance of the early-time (linear) 'shearing' mechanism of $\omega_{x}$ generation by STG (described in $\S 4.2$ ). Subsequently, as nonlinear effects (explained in $\S 5.2 .2$ ) become prominent, concentration of the $+\omega_{x}$ layer occurs on one streak flank, as formation of a new streamwise vortex commences (SP in figure $24 c$ ). The loss of $z$-reflectional symmetry in figure $24(c)$, initially present (at low perturbation amplitudes) due to symmetries in the linearized perturbation equations (see §3.1), signals the onset of significant nonlinearity. Note that the $\omega_{x}$ distribution at a half-wavelength in $x$ away (i.e. structure $\mathrm{SN}$ ) is obtained by $z$ reflection and sign inversion. As $\omega_{x}$ amplification continues, new collapsed streamwise vortices (i.e. with compact, near-circular cross-section) emerge on a timescale of $t^{+} \sim 50$ (figure $24 d$ ). This generation of new streamwise vortices from near-wall $\omega_{x}$ layers is strikingly similar to that noted in minimal channel flow (see $\mathrm{SH}$ ), and consistent with observations in other studies. However, contrary to prior speculations, the $\omega_{x}$ layers do not in reality roll up due to two-dimensional self-advection (discussed in $\S 5.2 .2$ ). Instead, the vor- 
(a)

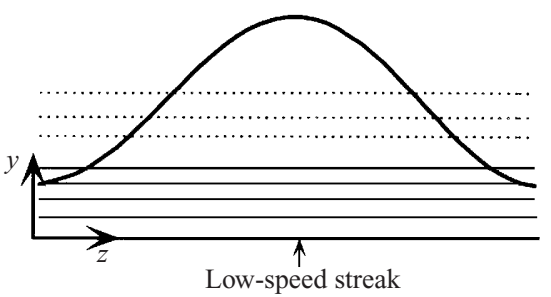

(c)

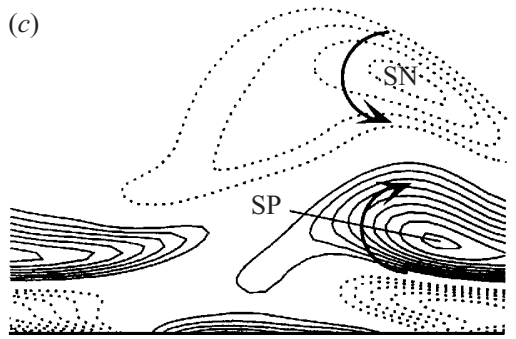

(b)
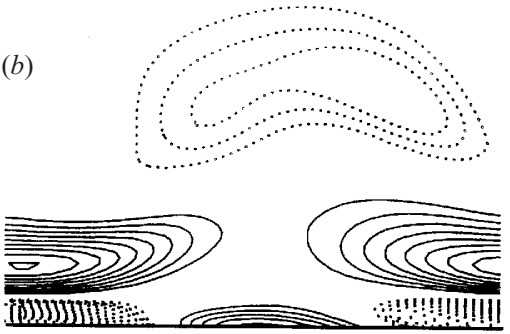

(d)

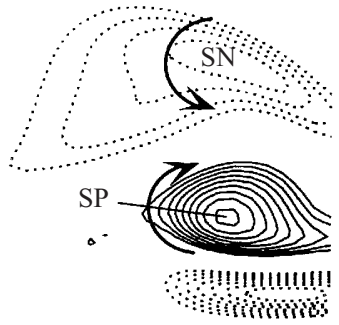

FiguRE 24. Streamwise vortex formation due to nonlinear transient growth, illustrated by cross-stream distributions of $\omega_{x}$ at $(a) t^{+}=0,(b) t^{+}=17,(c) t^{+}=35,(d) t^{+}=45$. The $(y, z)$-planes are tracked downstream with the $x$-phase of the STG perturbation, with a "phase speed' of approximately $0.6 U_{c}$.

(a)
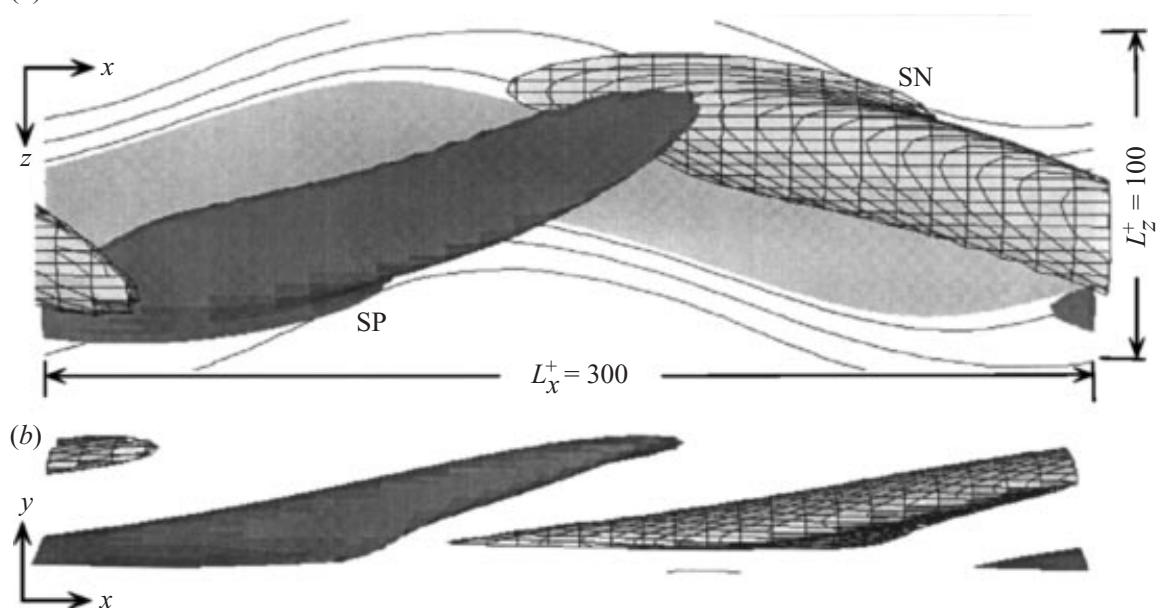

FIGURE 25. Streamwise vortices generated by STG of linearly stable streaks in $(a)$ top view, $(b)$ side view at $t^{+}=45$. Isosurfaces of $\omega_{x}$ at levels $+\left.0.6 \omega_{x}\right|_{\max }$ and $-\left.0.6 \omega_{x}\right|_{\max }$ are (dark) shaded and hatched, respectively; contours of $u$ at $y^{+}=20$ are shaded to indicate the low-speed streak.

tex generation mechanism is inherently three-dimensional, dominated by intense $\omega_{x}$ stretching. Note that past the initial (transient) vortex formation, streamwise vortices and hence turbulence are sustained indefinitely as the minimal channel regeneration cycle commences (figure 22), indicating the robustness of this STG mechanism.

The three-dimensional geometry of the new STG-generated vortices (i.e. SP and SN, with positive and negative $\omega_{x}$, respectively) is characterized by $x$-overlapping of tilted, opposite-signed streamwise vortices on either side of a low-speed streak (figure 25). 

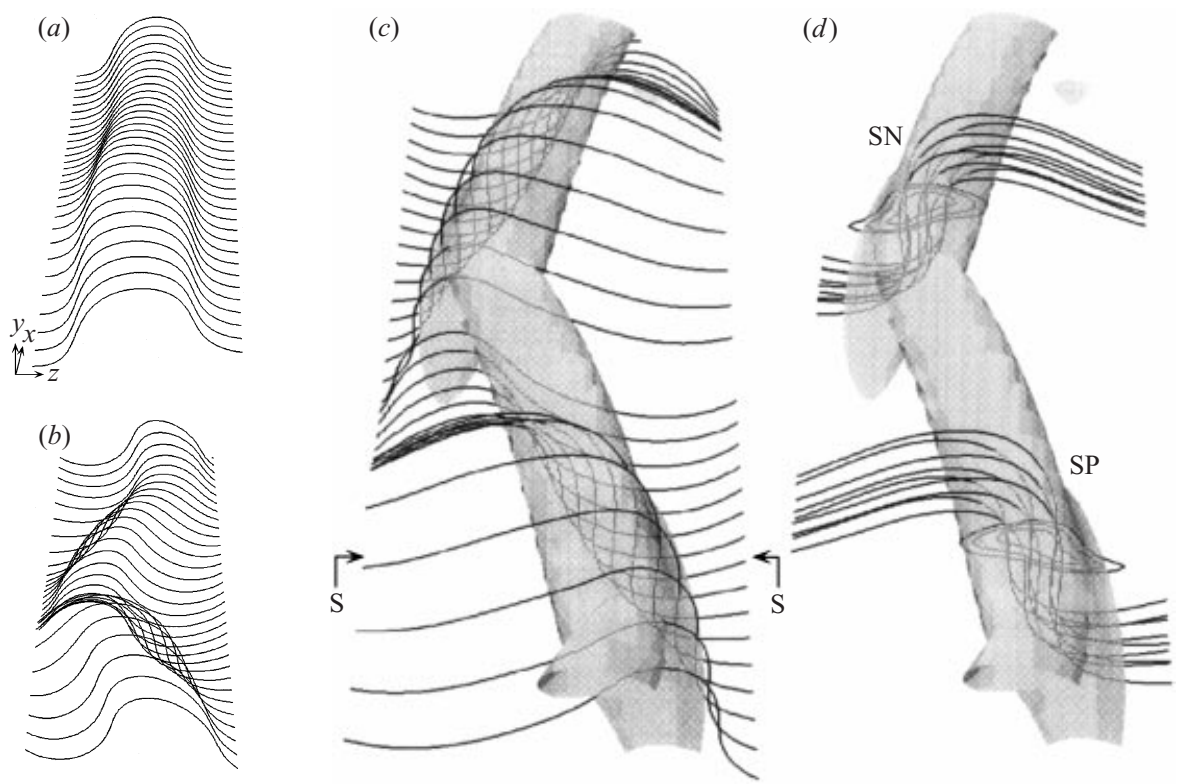

FIGURE 26. Vortex line geometry during transient growth-based vortex generation: $(a) t^{+}=0 ;(b)$ $t^{+}=17 ;(c, d) t^{+}=45$. THe vortex lines in $(a-c)$ are begun on an $x$-distributed rake within the streak trough region (i.e. $z=0$ ); the rake in $(d)$ consists of nine lines (in a square) passing through the core of the $x$-midplane of vortices SP and SN (shown as grey-shaded $\omega_{x}$ isosurface levels $+\left.0.6 \omega_{x}\right|_{\max }$ and $-\left.0.6 \omega_{x}\right|_{\max }$ in $(c)$ and $\left.(d)\right)$.

Note that the spatial relation of SP and SN is maintained upon evolution except for increasing overlap, with the vortex lengthening eventually arrested by viscous decay. Most significantly, this vortex geometry is strikingly similar to that of threedimensional coherent structures (CS) educed (from more than 100 vortex realizations) in fully developed near-wall turbulence (Jeong et al. 1997). Inherent non-uniformity of the base-flow streaks causes variations in vortices from one realization to another, and the finite-amplitude incoherent turbulence makes the instantaneous structures even more variant (see figure 1). Thus, ensemble averaging of a large number of base flow/perturbation combinations (i.e. CS eduction) is required to reveal the dominant vortex generation mechanism. The close correspondence of figure 25 with the ensemble-averaged CS (see also SH) serves as strong evidence that this STG-based vortex formation process is a dominant mechanism in near-wall turbulence.

Visualization of the corresponding vortex line geometry during STG-induced streamwise vortex formation is presented in figure 26, which displays a rake of vortex lines near the emerging SP and SN vortices. Note that the frames $(a),(b),(c)$ in figure 26 correspond to frames $(a),(b),(d)$ in figure 24 ; the latter are at the $x$-plane $\mathrm{S}-\mathrm{S}$ identified in figure $26(c)$. The initially $x$-aligned hairpin-shaped vortex lines (figure 26a) reflect the essentially straight and uniform initial streak, representative of the quiescent phase of minimal channel flow (Jimenez \& Moin 1991). Due to the STG $w(x)$ perturbation, the streak vortex lines exhibit a growing sinuous $z$-displacement (see $\S 4.3$ and figure 18), which reaches a significant (nonlinear) amplitude (figure $26 b$ ). Subsequently, vortex lines coalesce due to vortex stretching, resulting in new collapsed streamwise vortices SP and SN (figure 26c). Note that core dynamics associated with these finite-length streamwise vortices are countered by strong viscous effects near the wall. In figure $26(d)$, we show vortex lines initiated as a rake within the cores of 
SP and SN; clearly, these lines cross the corresponding $\omega_{x}$ surfaces, illustrating the limitation of vortex line tracing in vortex identification, particularly near the wall.

These results clearly have some important conceptual implications for vortex regeneration. Foremost, we have demonstrated that the presence of a strong existing vortex is unnecessary for new vortex generation, contrary to prior parent-offspring scenarios (reviewed in $\S 1$ ). Nevertheless, $w(x)$ perturbations of finite, yet moderate amplitude (e.g. $w_{r m s}^{+} \sim 0.5$ ) are required for vortex generation from the more numerous stable streaks considered here. Thus, pre-existing (parent) vortices do play an indirect role in generating the (relatively weak) spanwise velocity disturbance environment required for vortex generation. Note that since the STG mechanism delineated here causes simultaneous growth of both $+\omega_{x}$ (SP) and $-\omega_{x}$ (SN) vortices at different $x$, the evolution of SP and SN in fixed $(y, z)$-planes might superficially give the impression that one vortex is generating the other. In reality, both vortices originate and mature simultaneously from initially vortex-free streaks, as seen in figures 24-26.

\subsubsection{Evolutionary vortex dynamics}

Since the newly generated vortices SP and SN are predominantly streamwise (figure 25), further insight into the dynamics of near-wall vortex formation can be obtained by considering the inviscid evolution equation for $\omega_{x}$ :

$$
\frac{\partial \omega_{x}}{\partial t}=-u \frac{\partial \omega_{x}}{\partial x}-\underbrace{v \frac{\partial \omega_{x}}{\partial y}-w \frac{\partial \omega_{x}}{\partial z}}_{\text {Advection }}+\underbrace{\omega_{x} \frac{\partial u}{\partial x}}_{\text {Stretching }}+\underbrace{\frac{\partial v}{\partial x} \frac{\partial u}{\partial z}-\frac{\partial w}{\partial x} \frac{\partial u}{\partial y}}_{\text {Tilting }} .
$$

Note that the last two terms in (18) together represent the vortex line 'tilting' terms, rewritten to simplify their visual interpretation. Individually, each of the two terms constituting tilting indeed represents (instantaneously) the 'shearing' mechanism of $\omega_{x}$ generation explained in $\S 4.2$ and figure 16. In order to evaluate the role of the different terms in (18) in SP formation, we plot the distributions of each term in figure 27 at the instant of figure 24(c) (i.e. at $t^{+}=35$ ). By this time, the STG perturbation amplitude is sufficiently large to trigger nonlinear effects, leading to the onset of vortex generation. Note that the advection and stretching effects are necessarily nonlinear, as these terms do not appear in the linearized evolution equations for STG. In figure 27, the bold contour of $+\omega_{x}$ in $(a)-(d)$ provides common reference, denoting the emerging SP vortex boundary (same as in figure 24c). Generation terms which act to strengthen $+\omega_{x}$ and hence SP are indicated by positive magnitudes, whereas negative values indicate local weakening of SP.

Vorticity tilting. We find that significant $+\omega_{x}$ intensification occurs due to the generation term $(-\partial w / \partial x)(\partial u / \partial y)$ (figure 27c), which dominates the $(\partial v / \partial x)(\partial u / \partial z)$ term (figure 27b) near the wall. Note that in linear evolution, the $-(\partial w / \partial x)(\partial u / \partial y)$ term corresponds to the $\Omega \partial u_{s}^{\prime} / \partial x$ generation term, responsible for creation of a $\omega_{x}^{\prime}$ sheet (and $x$-circulation generation) in the streak trough region (figure $13 b$; see $\S 4.2$ ). In the nonlinear regime of STG, the $-(\partial w / \partial x)(\partial u / \partial y)$ term is largest in magnitude over all other terms, consistent with fully developed turbulence (Brooke \& Hanratty 1993). However, this term actually contributes to the thin tail ( $\mathrm{C}$ in figure $27 c$ ) of the near-wall $\omega_{x}$ layer, not to the main vortex (SP). Hence, the $-(\partial w / \partial x)(\partial u / \partial y)$ term is not responsible for vortex formation, as it contributes insignificant $+\omega_{x}$ within SP.

Two-dimensional advection. Prior studies (e.g. Sendstad \& Moin 1992; Brooke \& Hanratty 1993) have proposed that near-wall $\omega_{x}$ sheets (like those generated by STG) 'roll-up' due to their self-advection, an effect represented by the $\omega_{x}$ advection terms 
(a)

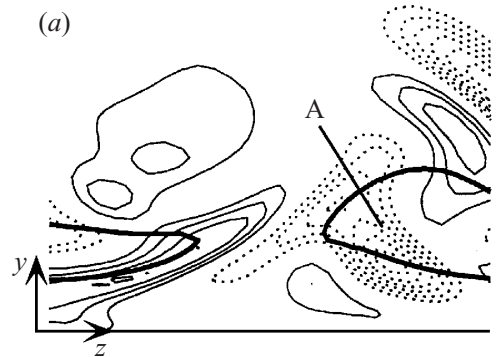

(c)

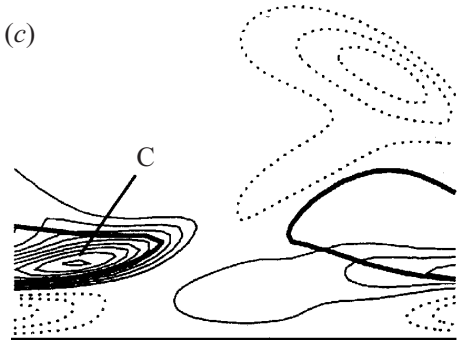

(b)

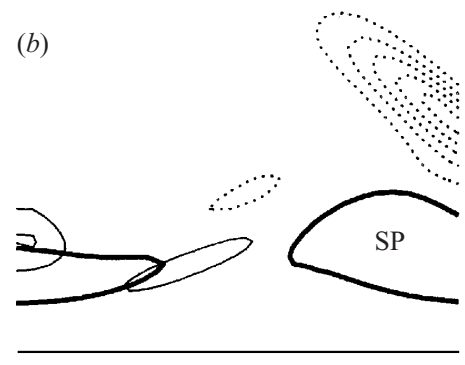

$(d)$

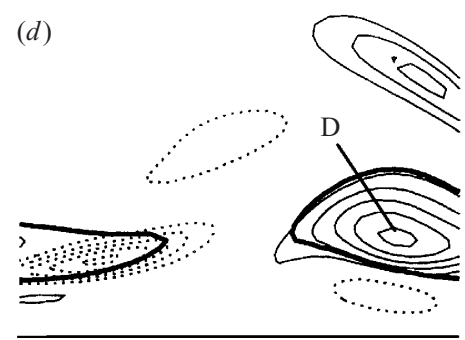

FIGURE 27. Distributions of terms of the $\omega_{x}$ evolution equation during STG-based vortex generation at $t^{+}=35:(a)$ two-dimensional $\omega_{x}$ advection $\left(-v \partial \omega_{x} / \partial y-w \partial \omega_{x} / \partial z\right),(b)$ the $(\partial v / \partial x)(\partial u / \partial z)$ generation term, $(c)$ the $-(\partial w / \partial x)(\partial u / \partial y)$ generation term, and $(d)$ direct stretching $\left(\omega_{x} \partial u / \partial x\right)$. The bold line in each panel identifies the $+\omega_{x}$ layer in figure $24(c)$.

(a)

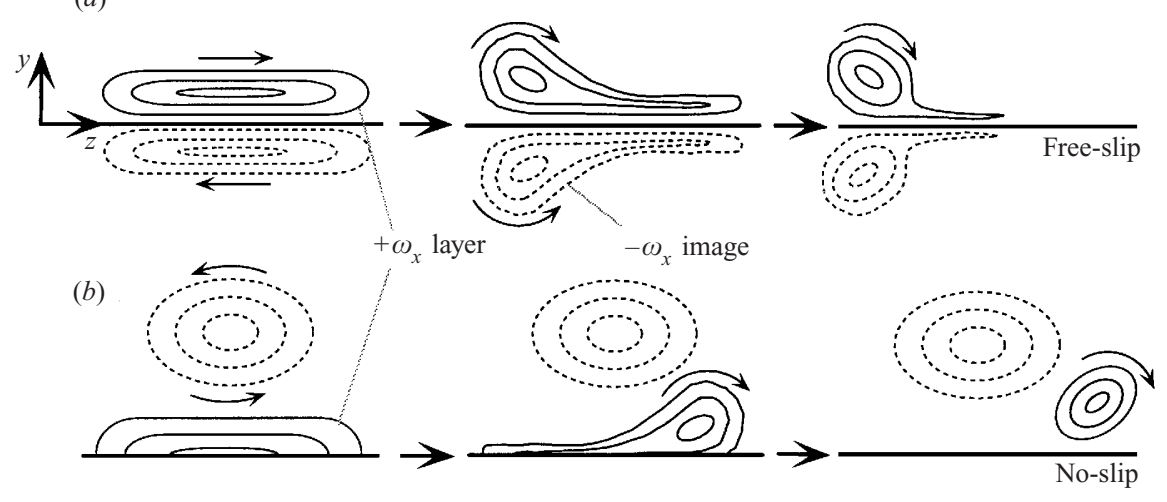

FIGURE 28. Schematic of two-dimensional rollup mechanisms of a near-wall $+\omega_{x}$ layer: $(a)$ rollup on the layer's left side near a free-slip wall due to image vorticity and 'head-tail' dipole formation by mutual induction, and $(b)$ rollup on the layer's right side near a no-slip due to ejection by an opposite-signed vortex $\left(-\omega_{x}\right.$ contours dashed) overhead.

in (18). In purely two-dimensional flow, near-wall vorticity sheets can roll up via two distinct mechanisms, illustrated in figure 28: (i) on the left side of a $+\omega_{x}$ layer above a free-slip wall, with dipole-like 'head-tail' formation due to the wall image vorticity (see Jimenez \& Orlandi 1993), as illustrated in the sequence in figure 28(a), or (ii) on the right side of a $+\omega_{x}$ layer attached to a no-slip wall, due to lifting of the wall-generated $\omega_{x}$ by a parent vortex, as in vortex wall-rebound (see Orlandi 1990 ), as shown by the sequence in figure $28(b)$. Note that for the case of a nearwall patch with opposite vorticity $\left(-\omega_{x}\right.$, each mechanism will lead to rollup on the opposite side of the layer from that shown in figure 28 ; i.e. roll up on the right side in figure 28( $a)$ and left side in figure $28(b)$. For the vortex formation studied 


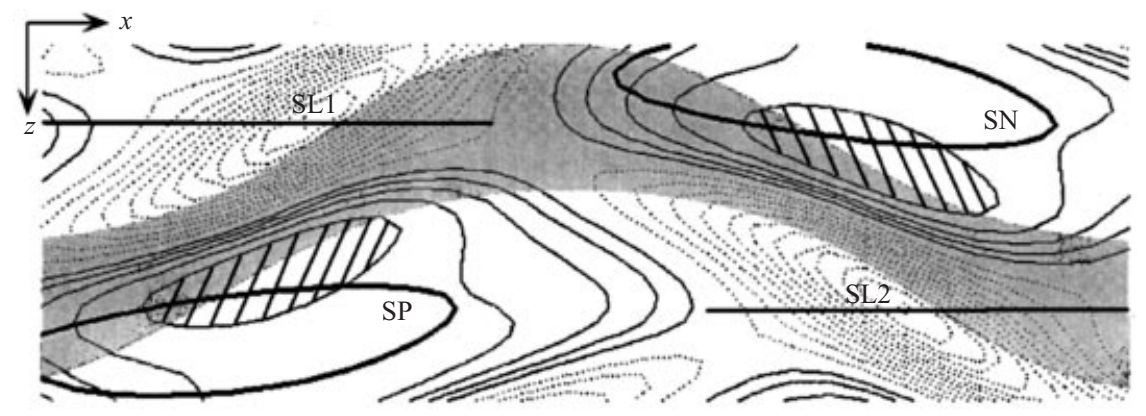

FIGURE 29. Generation of positive (solid) and negative (dotted) $\partial u / \partial x$ by STG-induced waviness of a streak (shaded) at $y^{+}=20$. The cross-section through the positive (SP) and negative (SN) streamwise vortices are denoted by solid bold contours of $\left|\omega_{x}\right|$. The hatched regions denote the largest values of vortex stretching by $+\partial u / \partial x$, while lines SL1 and SL2 identify internal shear layers containing $-\partial u / \partial x$.

here (as well as vortex formation typically observed in near-wall turbulence), SP forms on the left side of the $+\omega_{x}$ layer (figure 24c), hence indicating that the vortex rebound mechanism (ii) of $\omega_{x}$ sheet rollup plays no significant role. Even though mechanism (i) would also lead to vortex formation on the left side of the $+\omega_{x}$ layer (figure 28a), analysis of the $\omega_{x}$ advection terms during SP formation indicates that this image vorticity rollup mechanism is insignificant. In particular, $\omega_{x}$ advection actually opposes vortex formation, acting instead to reduce $+\omega_{x}$ (region A in figure 27a) where SP eventually forms. The negative amplitude of the advection term within SP indicates that the opposite-signed SN vortex immediately above the $+\omega_{x}$ layer (figure $24 c$ ) acts to advect $+\omega_{x}$ (i.e. SP) toward the right. Since SP eventually forms on the left side of the $+\omega_{x}$ layer (figure 24d), a mechanism other than $\omega_{x}$ advection must be responsible for vortex generation. As described below, a three-dimensional mechanism is actually responsible for vortex generation, dominating the competing (two-dimensional) advection effects. This lack of wall-induced vortex formation serves to explain the recent observation that elimination of the no-slip condition for $w$ (with a stress-free condition $\partial w / \partial y=0)$ actually enhances the near-wall turbulence intensity and drag (Jimenez \& Pinelli 1999).

Direct stretching. In reality, SP formation is dominated by direct stretching of $+\omega_{x}$, evident from nearly circular regions of $+\omega_{x} \partial u / \partial x$ (D in figure 27d) embedded within region SP. We find that this local $\omega_{x}$ stretching is sustained in time and is mainly responsible for the vortex collapse, whose location coincides with the $+\omega_{x} \partial u / \partial x$ peak. The $+\partial u / \partial x$ responsible for vortex collapse by stretching is a simple consequence of low-speed streak waviness, as illustrated by $\partial u / \partial x$ contours overlaid onto the wavy streak (figure 29). Recall that streak waviness is generated naturally by (initially linear) transient growth of the STG perturbation, via spanwise streak displacement by perturbation velocity normal to the streak flanks (described in $\S 4.3$ ). Once this waviness grows to a finite size, strong $+\partial u / \partial x$ develops downstream of the regions of maximum $z$-displacement (i.e. hatched regions in figure 29). Since a large velocity difference exists across the streak flanks (e.g. differential $U$ between high-speed and low-speed fluid at a fixed $y$ in figure $2 c$ ), a sizeable value of $+\partial u / \partial x$ is quickly generated once the streak flank is tilted in the $(x, z)$-plane by the growing streak waviness (recall the rapid temporal nature of STG). Consequently, direct stretching of positive and negative $\omega_{x}$ occurs in these regions of $+\partial u / \partial x$, which are the eventual locations of vortices SP and SN (bold $+\omega_{x}$ and $-\omega_{x}$ contours in figure 29). (Note 
(a)
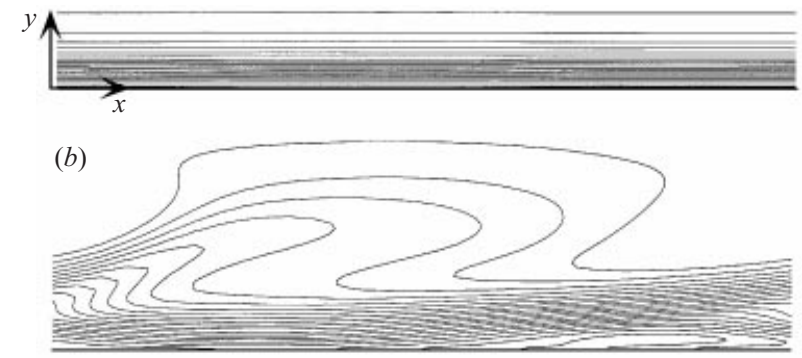

(c)

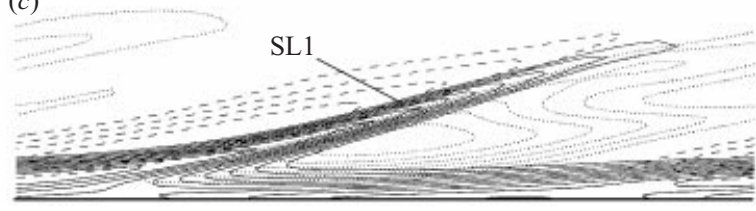

FIGURE 30. Formation of internal shear layer SL1 (figure 29) by STG, illustrated by $-\omega_{z}$ (solid) contours at $(a) t^{+}=0,(b) t^{+}=17$, and $(c) t^{+}=52$. Contours of positive (dashed) and negative (dotted) $u_{t}$ overlaid in $(c)$ illustrate the strong velocity gradient $\partial u / \partial x$ within SL1.

that positive and negative $\omega_{x}$ generation is maximum at points $\mathrm{P}$ and $\mathrm{N}$ respectively in figure $20 b$.) The initial near-wall $\omega_{x}$ sheets quickly collapse (figure $24 d$ ) due to this localized stretching (figure $27 d$ ), overcoming viscous diffusion which would otherwise cause their annihilation. Note that these dynamics are also captured as +VISA events (i.e. $+\partial u / \partial x$ ) existing within the core of the ensemble-averaged coherent structures (Jeong et al. 1997), further indicating the dominance of this STG-based vortex formation process.

\subsection{Internal shear layer generation}

Simultaneous with the generation mechanism of streamwise vortices outlined above, nonlinear evolution of STG also produces the well-known internal shear layers of spanwise vorticity. Internal shear layers are sharp, inclined $+u /-u$ interfaces in $(x, y)$ (e.g. with slope $\sim 30^{\circ}$ from $x$ ), and hence are characterized by intense both $\partial u / \partial y$ (hence $-\omega_{z}$ ) and $-\partial u / \partial x$. Prior studies indicate that internal shear layers are generated both by $x$-localized ejections (Bogard \& Tiederman 1987) and by waviness ('asymmetry') of elongated low-speed streaks in $(y, z)$ (Johansson et al. 1991). Internal shear layers are also detected well by the VITA conditional sampling technique, originally developed to detect 'bursts' via strong local $|\partial u / \partial x|$ events (Blackwelder \& Kaplan 1976). Here, we explain the genesis of internal shear layers, revealing close correspondence of the STG-generated internal shear layers to both experimental VITA detections and asymmetric, ensemble-averaged VISA events in DNS data.

During (nonlinear) STG, a pair of internal shear layers is created at locations SL1 and SL2 in figure 29, indicated by the generation of wall-detached layers of $-\omega_{z}$ (shown for SL1 in figure 30). In particular, the originally uniform $-\omega_{z}$ layer of the straight streak (figure $30 a$ ) is quickly transformed by the $z$-displacement of the lifted streak by the $w(x)$ perturbation of STG, resulting in creation of a tongue-shaped $-\omega_{z}$ region (figure $30 b$ ). Subsequently, this wall-detached $-\omega_{z}$ is significantly intensified into a thin, inclined shear layer (figure 30c), as commonly observed in $(x, y)$ snapshots of near-wall turbulence. Note the close resemblance of the fluctuation $+u_{t}$ and $-u_{t}$ contours in this instantaneous flow (figure $30 \mathrm{c}$ ) to the VISA-triggered ensemble 


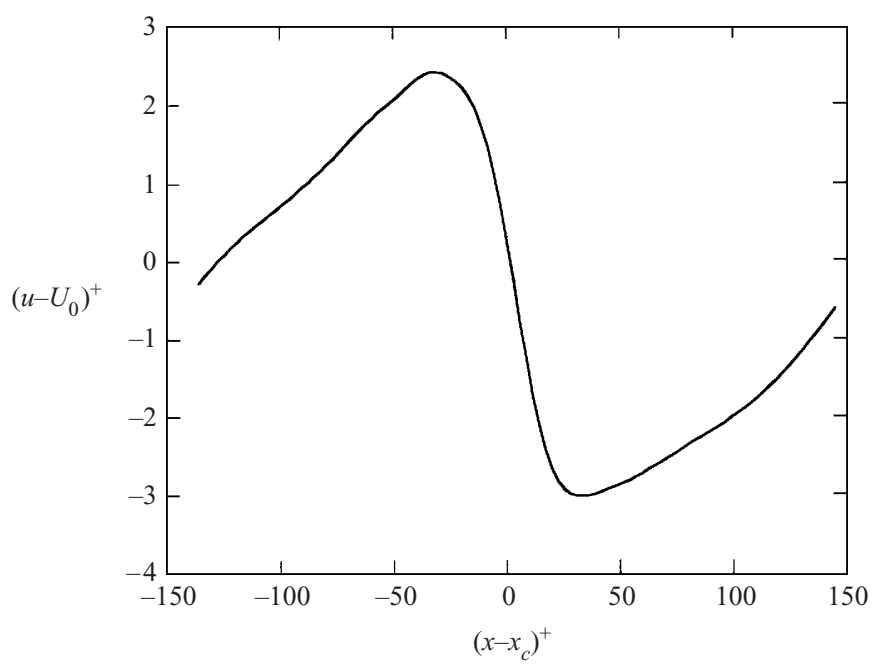

FIGURE 31. Trace of fluctuating velocity $u_{t}$ through the internal shear layer in figure $30(c)$ at $y^{+}=20$, illustrating a -VISA event centred at $x_{c}$, the midpoint of SL1.

average (Johansson et al. 1991), both in magnitude and contour shape (including the sharp $+u /-u$ interface). Furthermore, the $u_{t}$ trace through the internal shear layer at $y^{+}=20$ (figure 31 ), aligned with the minimum of $-\partial u / \partial x$ (i.e. a - VISA event), agrees well with experimental ensemble-averaged +VITA events. The asymmetry in $x$ of this trace clearly shows why +VITA (-VISA) events are more numerous for a given variance threshold. This generic feature of internal shear layers is a straightforward kinematic consequence of streamwise $u$ gradients (i.e. self-steepening of $-\partial u / \partial x$ by impact of high-speed $u$ with downstream low-speed $u$, and vice versa for $+\partial u / \partial x$ ), as explained in Jeong et al. (1997). Finally, note that by normalizing the data in figure 31 as $u_{t} / u_{r m s}$, a peak amplitude near unity is obtained, consistent with amplitudes of both (experimental) VITA and (DNS-based) VISA events.

Generation mechanism. Note that the internal shear layer formation occurs simultaneously with the creation of streamwise vortices SP and SN. As shown in figure 29, the internal shear layer SL1 (the line is the projection of the tongue in figure 30c) is generated directly across the streak (in $z$ ) from the simultaneously developing streamwise vortex SP. By symmetry, a second internal shear layer SL2 occurs across from $\mathrm{SN}$. Note that the initiation of internal shear layer formation commences at early times (e.g. at $t^{+}=17$, figure $30 b$ ), before streamwise vortex formation has occurred (figure 24b). Thus, the vortex and shear layer pairs are generated simultaneously, rather than sequentially, indicating that existing vortices are non-essential for internal shear layer formation. Note that $+\partial u / \partial x$ (responsible for vortex collapse) is naturally accompanied by $-\partial u / \partial x$ across a wavy streak (dotted contours in figure 29), constituting the sharp $+u /-u$ interface (i.e. with large $-\partial u / \partial x$ ) embedded within the internal shear layer (figure $30 \mathrm{c}$ ). Hence, generation of streak waviness by nonlinear STG is responsible for the simultaneous genesis of both streamwise vortices and internal shear layers, processes driven by $+\partial u / \partial x$ and $-\partial u / \partial x$, respectively.

Progressing further, we now explore the mechanism by which $-\partial u / \partial x$ (due to streak waviness) generates an intense, wall-detached shear layer of $\partial u / \partial y$ and $-\omega_{z}$ (figure $30 \mathrm{c}$ ). As illustrated in figure 32, the internal shear layer forms on the flank of the lifted streak, where high-speed fluid impacts the wavy low-speed streak (hence 


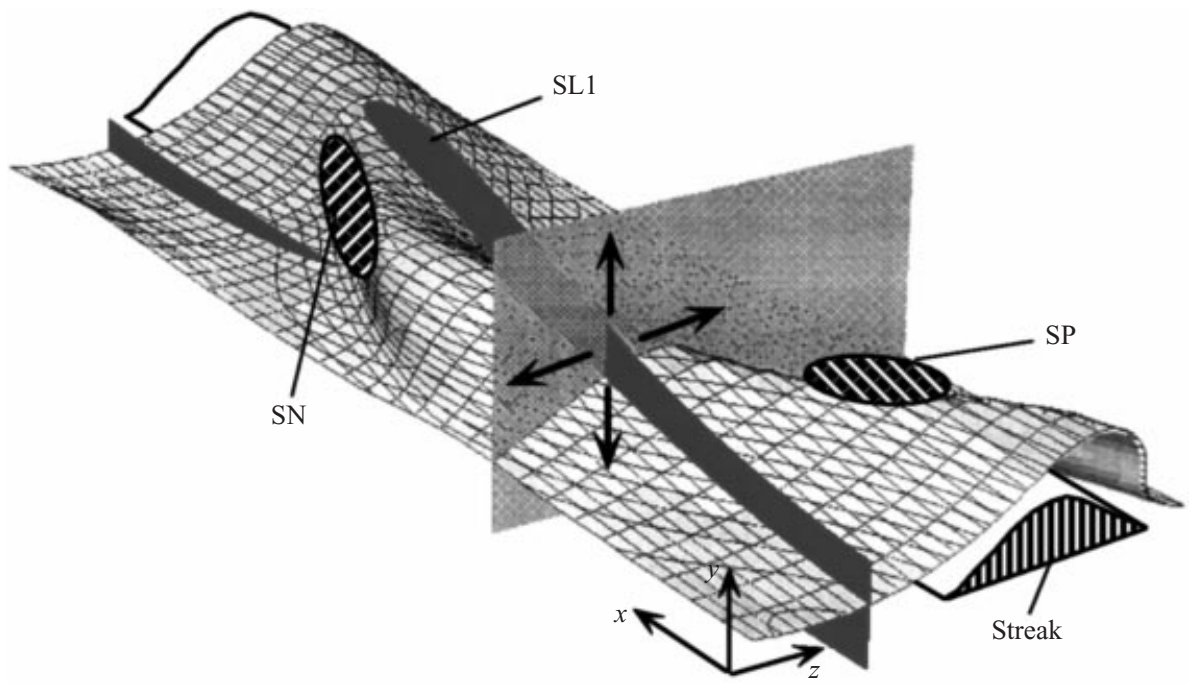

FIGURE 32. Relation of internal shear layer SL1 (figure 29) to the STG-perturbed streak. The black region of high $\left|\omega_{z}\right|$ marks the mid-plane of SL1, and the wavy streak is underneath the lifted $u=0.5 U_{c}$ surface (hatched). The relative locations of the streak and structures SP and SN are added for illustrative purpose. The local stagnation flow (illustrated by arrows) near the shaded $(y, z)$-plane is addressed in figure 33 .

(a)

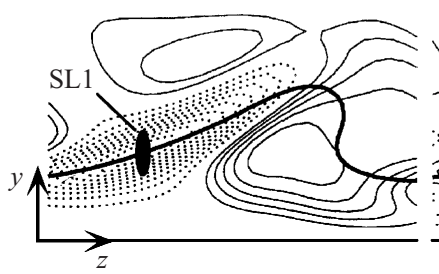

(b)

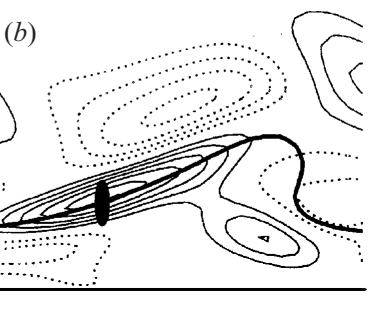

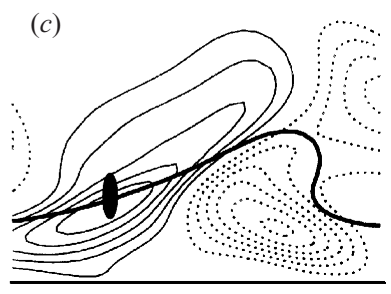

FIGURE 33. Distributions of normal strains (a) $\partial u / \partial x,(b) \partial v / \partial y$, and (c) $\partial w / \partial z$ in the $(y, z)$ cross-section shaded in figure 32, elucidating the extensional strain in the $(y, z)$-plane responsible for internal shear layer generation (positive and negative contours solid and dashed, respectively).

generating $-\partial u / \partial x$ at the hatched plane). The hatched plane is drawn at the $x$ location of minimum $-\partial u / \partial x$ (maximum negative), and the velocity gradients in this plane are shown in figure 33. Being associated with a stagnation-type flow in $x$, $-\partial u / \partial x$ (figure $33 a$ ) produces a source-type flow in the hatched plane, resulting in large values of both $+\partial v / \partial y$ (figure $33 b$ ) and $+\partial w / \partial z$ (figure $33 c$ ) on the streak flank. Consequently, any pre-existing vorticity $\left(\omega_{y}\right.$ and $\left.\omega_{z}\right)$ along the streak flank is stretched by this extensional strain. Hence, the internal shear layers observed here are composed of streak-flank vorticity locally displaced in $z$ toward the $(x, y)$-plane of SL1 (by STG of the streak wave), and stretched by the (kinematically required) extensional strain. That is, where a streamwise vortex (SP or $\mathrm{SN}$ ) consisting of predominantly $\omega_{x}$ is formed on one flank of a streak, an internal shear layer (predominantly $\omega_{z}$ ) forms on the other flank of the streak. In contrast to a scenario of localized ejection, this internal shear layer generation mechanism is inherent to a wavy streak, and does not require advection or stretching by existing vortices. 


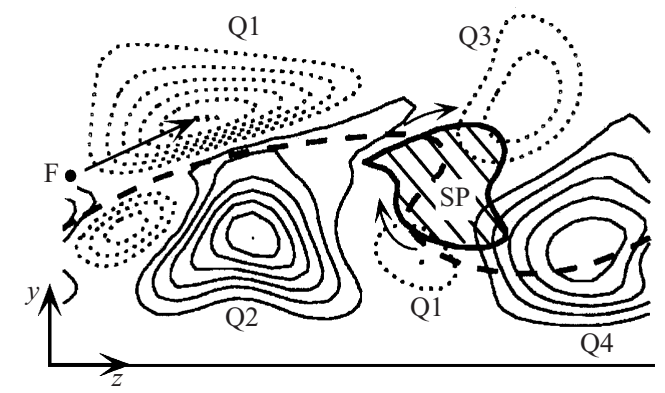

FiguRE 34. Instantaneous quadrant $-u v$ Reynolds stress events (Q1, Q2: ejection, Q3, and Q4: sweep) generated by STG at $t^{+}=45$. The low-speed streak is denoted as the $u=0.5 U_{c}$ contour (bold-dashed), and the (positive) streamwise vortex SP is indicated by the $+\left.0.6 \omega_{x}\right|_{\max }$ contour (hatched).

\subsection{Reynolds stress and turbulence statistics}

To further quantify the role of nonlinear STG in near-wall turbulence, we also consider the generation of $-u v$ Reynolds stress events and corresponding fluctuation amplitudes. In the context of the $u v$-quadrant-based conditional averaging procedure developed by Wallace, Eckelmann \& Brodkey (1972), a representative distribution of instantaneous $-u v$ events is shown in a $(y, z)$ cross-section in figure 34 . Near the newly generated SP vortex (identified by shading), the distribution of quadrant Reynolds stresses agrees well with the educed coherent structure (Jeong et al. 1997), including the location of ejection (Q2), sweep (Q4), and counter-gradient stresses (Q1, Q3). Interestingly, a region of significant counter-gradient Reynolds stress (Q1) occurs at larger $y$ away from the vortex. Due to the $y$-variation of the $w$ perturbation of STG, lowspeed fluid is displaced in $z$ underneath high-speed fluid overhead. In the $(y, z)$-plane, this effect, along with the induction of both SP and SN, produces $z$-asymmetry of the streak back (i.e. with the left side flattened). The sliding of the fluid element $F$ from the high-speed region up the streak back (marked by a thick dashed line in figure 34) is associated with both $+u$ and $+v$, hence Q1. DNS flow visualization reveals that this Q1 event occurs throughout the early-stages of STG-induced vortex formation, and thus may be useful as an indirect indicator of STG operation in snapshots of nearwall turbulence. At later times, the Q1 magnitude decreases as the newly generated vortex modifies (through ejection) the original streak from which it was formed.

The net $(x, z)$-averaged Reynolds stress at $y^{+}=20$ indicates a rapid increase in turbulence production due to STG (figure 35). Whereas the Reynolds stress is initially identically zero, transient growth quickly generates (by $t^{+} \sim 80$ ) mean values approaching that in fully developed turbulence $\left(0.65\right.$ at $y^{+}=20$, Kim et al. 1987), with even larger values for a perturbation amplitude of $w_{r m s}^{+}=0.9$ (figure 35b). Consistent with the well-known role of streamwise vortices, the growth of turbulence production results from the $v$-induction of newly generated streamwise vortices, captured statistically by $v_{r m s}$ and $\omega_{x r m s}$, respectively (figure 35 ). Note that starting from a quiescent initial flow, the amplitudes in fully developed turbulence $\left(v_{r m s}^{+}=0.55\right.$ and $\omega_{x r m s}^{+}=0.14$ at $y^{+}=20$ ) are approached to within $40 \%$ for $w_{r m s}^{+}=0.5$ (figure $35 a$ ) and fully attained for $w_{r m s}^{+}=0.9$ (figure $35 \mathrm{~b}$ ). Additionally, the amplitude of $v_{r m s}^{+}$in figure $35(b)$ agrees well with that observed in minimal channel flow turbulence (Jimenez \& Moin 1991). Thus, starting from a quiescent, laminar streak flow, the STG mechanism generates the turbulence levels observed in both minimal-domain and fully developed near-wall turbulence. 


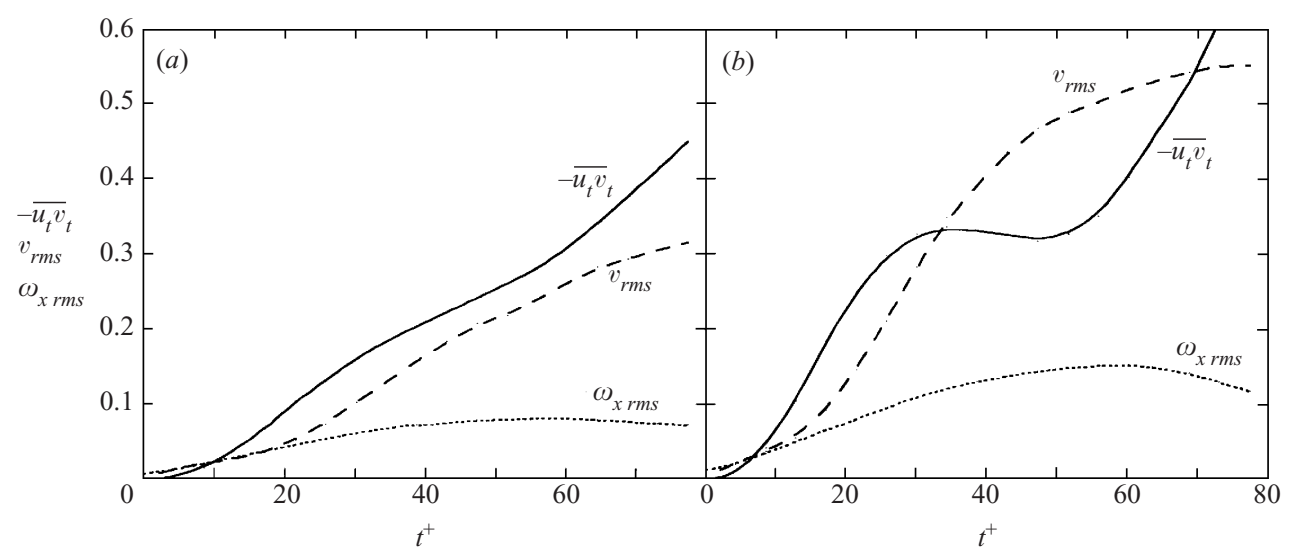

FIGURE 35. Growth of $(x, z)$-averaged turbulence statistics at $y^{+}=20$ due to STG: Reynolds stress $-u_{t} v_{t}$, normal velocity $v_{r m s}$, and streamwise vorticity $\omega_{x r m s}$. All quantities are wall-unit normalized, and the initial perturbation amplitude is $(a) w_{r m s}^{+}=0.5$ and $(b) w_{r m s}^{+}=0.9$.

\section{Concluding discussion}

Herein, we reveal a significant limitation of vortex (re)generation scenarios based on normal-mode streak instability. Namely, the streak-flank normal vorticity-shown here to critically determine the onset and growth rate of sinuous streak instability - is annihilated by viscous self-diffusion (i.e. planar vortex reconnection). Consequently, even strong, initially unstable streaks (shown here to constitute about $20 \%$ of bufferlayer streaks in fully developed turbulence) are stabilized on a rapid timescale of $t^{+} \sim 50$, limiting normal-mode growth to only a factor of two. These results serve as strong evidence that normal-mode streak instability does not contribute significantly to vortex generation and turbulence production near the wall.

As an alternative, we identify here a new streak transient growth (STG) mechanism, which generates an order-of-magnitude amplification of perturbation energy in $x$ dependent modes, even for more numerous, weaker, normal-mode-stable streaks. Compared to normal-mode instability, the STG mechanism is seen to be significantly more energetic and to operate more frequently (involving more streak regions) in a given flow volume. Owing to the temporal nature of transient growth for stable streaks (more typical of fully developed turbulence), the initial disturbance amplitude must be sufficient for significant nonlinear effects to be triggered (i.e. disturbances eventually decay linearly otherwise). We find that $w^{\prime}(x)$ perturbations of moderately low amplitude (e.g. $w_{r m s}^{+}=0.5$ ) do in fact lead to generation of new vortices and sustained near-wall turbulence.

Note that the STG perturbation (13) does not necessarily capture the optimal growth, as it reflects a simple idealization of $u w$ Reynolds stress events observed in near-wall turbulence. Analysis of quadrant 2 and $3 u w$ Reynolds stress magnitudes in fully developed turbulence-representative of the combination of $w(x)$ and lowspeed streaks required for transient growth - indicates that virtually all regions along streaks exhibit $u w$ magnitudes sufficient for subsequent STG to reach nonlinear amplitude. Note that the $w(x)$ observed within low-speed streaks reflects the induction of pre-existing vortices overhead, constituting the 'parent-offspring' component of the STG-based vortex generation scenario. Interestingly, near-wall turbulence exhibits a well-defined, $x$-alternating pattern of Q2 and Q3 uw Reynolds stresses, indicating that 
$u w$ events (and the STG mechanism this pattern captures) are thus a critical trigger for turbulence production.

Most importantly, transient growth into the nonlinear regime generates new collapsed streamwise vortices from normal-mode-stable streaks. Unlike prior suggestions, vortex generation does not involve streamwise vorticity generation at the wall (by the no-slip condition) or vorticity layer rollup-both two-dimensional mechanisms. Instead, the vortex formation is inherently three-dimensional, with direct stretching (inherent to streak $(x, z)$-waviness) of near-wall $\omega_{x}$ sheets leading to streamwise vortex collapse. Significantly, STG produces new collapsed streamwise vortices near the wall which closely resemble the ensemble-averaged CS (Jeong et al. 1997). The formation mechanism of internal shear layers (and associated VISA events) is also captured, along with quadrant Reynolds stresses and other turbulence statistics, all of which are shown to agree well with fully developed turbulence. In summary, evidence suggests the dominance of this STG-based vortex generation mechanism in near-wall turbulence.

The rapid temporal growth accompanying (nonlinear) STG suggests reassessment of the revised view of 'bursting'. In particular, the modern, widely accepted explanation of 'bursting' is the advection of spatially distributed streamwise vortices and related structural features past a fixed measurement or observation location (Robinson 1991). However, the STG-based vortex formation scenario described here does in fact reveal burst-like growth of $-u v, v$, and $\omega_{x}$ in time (in an advecting reference frame), in the spirit of the original temporal 'bursting' concept (Kline et al. 1967). Note that fixed-point measurements (including conditional sampling) would not clearly detect such temporal evolution, due to advection of the developing flow. The rapid temporal STG scenario we find suggest that both views - spatial advection of existing structures and temporal growth of new structures - are relevant to 'bursting'.

In the context of the related issue of bypass transition from the mean velocity $U(y)$, the present results suggest a two-step (linear) mechanism: (i) transient growth of $x$-independent perturbations of $U(y)$ to generate a finite-amplitude $z$-varying streak $U(y, z)$ via the 'lift-up' streak formation mechanism (i.e. prior transient growth analysis of $U(y)$ ), followed by (ii) STG of $x$-dependent perturbations of the streak $U(y, z)$ to generate (new) streamwise vortices and hence sustained turbulence. Alternatively, a single-step (linear) mechanism may also exist, involving a single $(x, z)$-dependent perturbation which simultaneously excites mechanisms (i) and (ii) from the mean velocity $U(y)$. That is, a wavy streak can form in the first place (via wavy lift-up by a sinuous perturbation) from a ((x,z)-homogeneous) laminar profile-as opposed to the scenario of formation of an $x$-homogeneous streak subsequently perturbed by a sinuous $w(x)$ (studied here). The measure of transient growth-used in the corresponding variational problem to quantify optimal perturbation growth-must then be specifically chosen to capture this single-step bypass mechanism. In particular, an appropriate perturbation measure should capture simultaneously the growth of the streak magnitude $\left(u^{\prime}\right)$ and its sinuous spanwise displacement $\left(w^{\prime}\right)$. Otherwise, consideration of perturbation kinetic energy $E$ identifies $x$-independent perturbations as optimal. This suggests use of a $u^{\prime} w^{\prime}$ Reynolds stress measure of transient growth, rather than $E$ as commonly used to date.

Our results also open many important, currently unexplored avenues for modelling and control of near-wall turbulence. For instance, the low-speed streaks in actual flows are certainly non-uniform, wavy, and surrounded by a sea of finite-amplitude perturbations or incoherent turbulence, in contrast to the clean flow we have analysed. Particularly for turbulence modelling (including near-wall modelling for LES), such 
instantaneous irregularities, including non-equilibrium outer flow conditions, require a more statistical framework for the mechanistic descriptions here. For instance, a promising approach is a triple decomposition of the fully turbulent flow into (i) (local) $x$ mean streaks $U(y, z)$, (ii) the (evolving) streak perturbation capturing transient growth, vortex generation and decay, and (iii) incoherent turbulence. The fact that the ensemble-averaged (over numerous irregular structures) CS and the STG-generated (instantaneous) vortices are very similar is encouraging, in that it suggests the mechanism described here is the underlying one.

Additionally, the association of vortex formation with an instability is promising from a control standpoint, noting the success of instability control in free shear flows (e.g. see Hussain 1983). The most logical approach to CS-based drag reduction and heat transfer suppression is to simply prevent vortex (re)generation in the first place (in contrast to popular approaches which counteract fully developed CS). Our focus is on large-scale control, wherein numerous streaks may be simultaneously stabilized by a single large-scale forcing. Based on this control premise, we have developed new techniques for drag reduction, enabling large-scale flow forcing without requiring instantaneous flow information (Schoppa \& Hussain 1998b). As proof-of-principle, we find that an $x$-independent forcing, with a wavelength of 400 wall units and an amplitude of only $6 \%$ of the centerline velocity, produces a significant sustained drag reduction: $20 \%$ for imposed counter-rotating streamwise vortices and $50 \%$ for colliding, $z$-directed wall jets. The drag reduction results from weakened longitudinal vortices near the wall, due to control-induced $\omega_{y}$ reduction, which in turn arrests the streak perturbation growth responsible for vortex generation. These results suggest promising new strategies for drag and heat transfer control, e.g. passive vortex generators or colliding spanwise jets from $x$-aligned slots, involving large-scale (hence more durable) actuation and requiring no wall sensors or control logic. For further details of these large-scale control strategies, see Schoppa \& Hussain $(1997,1998 b)$.

This research was supported by AFOSR grant F49620-97-1-0131, and NASA Graduate Fellowship grant NGT-51022 for W.S. F. H acknowledges support during the 1999 Turbulence Program at the Isaac Newton Institute, Cambridge, UK and the 2000 Hydrodynamic Turbulence Program at the Institute for Theoretical Physics, UCSB. Supercomputer time was provided by the NASA Ames Research Center. The authors gratefully acknowledge Professors J. Kim and R. Moser for providing the channel flow simulation code and turbulence database.

\section{REFERENCES}

Adrian, R. J., Meinhart, C. D. \& Tomkins, C. D. 1999 Vortex organization in the outer region of the turbulent boundary layer. TAM Rep. 924, University of Illinois, Urbana-Champagne.

Benney, D. J. 1961 A non-linear theory for oscillations in a parallel flow. J. Fluid Mech. 10, 209.

Benney, D. J. \& Gustavsson, L. H. 1981 A new mechanism for linear and nonlinear hydrodynamic instabilities. Stud. Appl. Maths 64, 185.

Bernard, P. S., Thomas, J. M. \& Handler, R. A. 1993 Vortex dynamics and the production of Reynolds stress. J. Fluid Mech. 253, 385.

BlaCKWELDER, R. F. \& KAPLAN, R. E. 1976 On the wall structure of the turbulent boundary layer. J. Fluid Mech. 76, 89.

Bogard, D. G. \& Tiederman, W. G. 1987 Characteristics of ejections in turbulent channel flow. J. Fluid Mech. 179, 1.

Brooke, J. W. \& Hanratty, T. J. 1993 Origin of turbulence-producing eddies in a channel flow. Phys. Fluids A 5, 1011. 
Brown, G. L. \& Thomas, A. S. W. 1977 Large structure in a turbulent boundary layer. Phys. Fluids 20, 5243 .

Butler, K. M. \& FARrell, B. F. 1992 Three-dimensional optimal perturbations in viscous shear flow. Phys. Fluids A 4, 1637.

Cantwell, B. J. 1981 Organized motion in turbulent flow. Annu. Rev. Fluid Mech. 13, 457.

Doligalski, T. L. \& WalkeR, J. D. A. 1984 The boundary layer induced by a convected twodimensional vortex. J. Fluid Mech. 139, 1.

GAD-EL-HAK, M. \& Hussain, F. 1986 Coherent structures in a turbulent boundary layer. Part 1: Generation of 'artificial' bursts. Phys. Fluids 29, 2124.

Haidari, H. A. \& SMith, C. R. 1994 The generation and regeneration of single hairpin vortices. J. Fluid Mech. 227, 135.

Hall, P. \& Horseman, N. J. 1991 The linear inviscid secondary instability of longitudinal vortex structures in boundary layers. J. Fluid Mech. 232, 357.

Hamilton, J. M., Kim, J. \& WalefFe, F. 1995 Regeneration mechanisms of near-wall turbulence structures. J. Fluid Mech. 287, 317.

Hussain, F. 1983 Coherent structures - reality and myth. Phys. Fluids 26, 2816

JANG, P. S., BENNey, D. J. \& GRAN, R. L. 1986 On the origin of streamwise vortices in a turbulent boundary layer. J. Fluid Mech. 169, 109.

JEONG, J. \& Hussain, F. 1992 Coherent structure near the wall in a turbulent channel flow. In Proc. Fifth Asian Congress of Fluid Mech., Taejon, Korea (ed. K. S. Chang \& H. Choi), p. 1262.

JeOng, J. \& Hussain, F. 1995 On the identification of a vortex. J. Fluid Mech. 285, 69.

JeOng, J., Hussain, F., Schoppa, W. \& Kim, J. 1997 Coherent structures near the wall in a turbulent channel flow. J. Fluid Mech. 332, 185.

Jimenez, J. \& Moin, P. 1991 The minimal flow unit in near-wall turbulence. J. Fluid Mech. 225, 213.

Jimenez, J. \& ORLandi, P. 1993 The rollup of a vortex layer near a wall. J. Fluid Mech. 248, 297.

Jimenez, J. \& Pinelli, A. 1999 The autonomous cycle of near-wall turbulence. J. Fluid Mech. 389, 335.

Johansson, A. V., Alfredsson, P. H. \& Kim, J. 1991 Evolution and dynamics of shear layer structures in near-wall turbulence. J. Fluid Mech. 224, 579.

KIM, J. 1987 Evolution of a vortical structure associated with the bursting event in a channel flow. Turbulent Shear Flows 5. Springer.

KIM, J., Moin, P. \& Moser, R. D. 1987 Turbulence statistics in fully developed channel flow at low Reynolds number. J. Fluid Mech. 177, 133.

Kline, S. J., Reynolds, W. C., Schraub, F. A. \& Rundstadler, P. W. 1967 The structure of turbulent boundary layers. J. Fluid Mech. 30, 741.

Kovasznay, L. S. G., Kibens, V. \& Blackwelder, R. F. 1970 Large-scale motion in the intermittent region of a turbulent boundary layer. J. Fluid Mech. 41, 283.

Lamb, H. 1945 Hydrodynamics. Dover.

Lin, S. J. \& Concos, G. M. 1984 The mixing layer: deterministic models of a turbulent flow. Part 3. The effect of plane strain on the dynamics of streamwise vortices. J. Fluid Mech. 141, 139.

Melander, M. V. \& Hussain, F. 1988 Cut-and-connect of two antiparallel vortex tubes. CTR Rep. S-21, p. 257.

Moin, P., Leonard, A. \& Kim, J. 1986 Evolution of a curved vortex filament into a vortex ring. Phys. Fluids 29, 955.

OrLandi, P. 1990 Vortex dipole rebound from a wall. Phys. Fluids A 2, 1429.

OrsZAG, S. A. 1971 Accurate solution of the Orr-Sommerfeld stability equation. J. Fluid Mech. 50, 689.

Panton, R. L. 1997 Self-sustaining Mechanisms of Wall Turbulence. Computational Mechanics Publications.

Panton, R. L. 2001 Self-sustaining mechanisms in turbulent boundary layers. Prog. Aero. Sci. (to appear).

Phillips, W. R. C., Wu, Z. \& Lumley, J. L. 1996 On the formation of longitudinal vortices in a turbulent boundary layer over wavy terrain. J. Fluid Mech. 326, 321.

RaO, K. N., Narasimha, R. \& Narayanan, M. A. 1971 The bursting phenomenon in a turbulent boundary layer. J. Fluid Mech. 48, 339. 
Reddy, S. C. \& Henningson, D. S. 1993 Energy growth in viscous channel flow. J. Fluid Mech. 252, 209.

ReYnolds, W. C. \& Hussain, F. 1972 The mechanics of an organized wave in turbulent shear flow. Part 3. Theoretical models and comparisons with experiments. J. Fluid Mech. 54, 263.

Robinson, S. K. 1991 Coherent motions in the turbulent boundary layer. Annu. Rev. Fluid Mech. 23, 601.

Schoppa, W. \& Hussain, F. 1997 Genesis and dynamics of coherent structures in near-wall turbulence. In Self-sustaining Mechanisms of Wall Turbulence (ed. R. Panton), p. 385. Computational Mechanics Publications (refered to here in as $\mathrm{SH}$ ).

Schoppa, W. \& Hussain, F. 1998 a Formation of near-wall streamwise vortices by streak instability. AIAA Paper 98-3000.

SCHOPPA, W. \& Hussain, F. $1998 b$ A large-scale control strategy for drag reduction in turbulent boundary layers. Phys. Fluids 10, 1049.

Schoppa, W., Hussain, F. \& Metcalfe, R. W. 1995 A new mechanism of small-scale transition in a plane mixing layer: core dynamics of spanwise vortices. J. Fluid Mech. 298, 23.

Sendstad, O. \& Moin, P. 1992 The near wall mechanics of three dimensional turbulent boundary layers. Rep. TF- 57, Stanford University.

Singer, B. A. 1996 Characteristics of a young turbulent spot. Phys. Fluids 8, 509.

Smith, C. R. \& Walker, J. D. A. 1994 Turbulent wall-layer vortices. In Fluid Vortices (ed. S. Green). Springer.

Swearingen, J. D. \& Blackwelder, R. F. 1987 The growth and breakdown of streamwise vortices in the presence of a wall. J. Fluid Mech. 182, 225.

Theodorsen, T. 1952 Mechanism of turbulence. In Proc. Second Midwestern Conf. of Fluid Mechanics. Ohio State University, p. 1.

Townsend, A. A. 1956 The Structure of Turbulent Shear Flows. Cambridge University Press.

Trefethen, L. N., Trefethen, A. E., Reddy, S. C. \& Driscoll, T. A. 1993 Hydrodynamic stability without eigenvalues. Science 261, 578 .

WALEFFE, F. 1995 Hydrodynamic stability and turbulence: Beyond transients to a self-sustaining process. Stud. Appl. Maths 95, 319.

WaLefFe, F. 1997 On a self-sustaining process in shear flows. Phys. Fluids 7, 3060.

WalefFe, F., Kim, J. \& Hamilton, J. M. 1993 On the origin of streaks in turbulent shear flows. In Turbulent Shear Flows 8 (ed. F. Durst et al.), p. 37. Springer.

Wallace, J. M., Eckelmann, H. \& Brodkey, R. S. 1972 The wall region in turbulent shear flow. J. Fluid Mech. 54, 39.

Yu, X. \& LiU, J. T. C. 1991 On the secondary instability in Gortler flow. Phys. Fluids A 3, 1845.

Zhou, J., Adrian, R. J., Balachandar, S. \& Kendall, T. M. 1999 Mechanisms for generating coherent packets of hairpin vortices in channel flow. J. Fluid Mech. 387, 353. 